UNIVERSIDADE DE SÃO PAULO

INSTITUTO DE FÍSICA

\title{
Buracos Negros em Universos Brana com Constante Cosmológica
}

\author{
Juliano Cesar Silva Neves
}

Orientador: Prof. Dr. Carlos Molina Mendes

Dissertação apresentada ao Instituto de Física da Universidade de São Paulo para a obtenção do título de Mestre em Ciências.

Comissão Examinadora:

Prof. Dr. Carlos Molina Mendes (EACH-USP)

Prof. Dr. George Emanuel Avraam Matsas (IFT-UNESP)

Prof. Dr. Rogério Rosenfeld (IFT-UNESP)

São Paulo 


\title{
FICHA CATALOGRÁFICA \\ Preparada pelo Serviço de Biblioteca e Informação do Instituto de Física da Universidade de São Paulo
}

\author{
Neves, Juliano César Silva \\ Buracos negros em universos brana com constante \\ cosmológica - São Paulo - 2008 \\ Dissertação (Mestrado) - Universidade de São Paulo. \\ Instituto de Física - Depto. de Física Matemática \\ Orientador: Prof. Dr. Carlos Molina Mendes \\ Área de Concentração: Física \\ Unitermos: 1. Buracos Negros; 2. Mundos Brana; \\ 3. Dimensões Extras; 4. Relatividade.
}

USP /IF/SBI-070/2008 


\section{Resumo}

Obtivemos famílias de soluções estáticas e esfericamente simétricas para buracos negros numa brana com vácuo, constante cosmológica não nula e imersa num bulk 5-dimensional assintoticamente anti-de Sitter. Para o caso das geometrias assintoticamente de Sitter, o único membro da família obtida foi a geometria Schwarzschild-de Sitter extrema. Para o caso de geometrias assintoticamente anti-de Sitter, obtivemos toda uma família de soluções, onde cada elemento desta família é determinado por uma constante $C$. Estudamos o comportamento destas soluções próximo e longe do horizonte de eventos. A seguir, analisamos a evolução de um campo escalar não massivo no exterior dos buracos negros obtidos. Verificamos analiticamente e numericamente o comportamento do potencial efetivo em função da coordenada tartaruga próximo ao horizonte de eventos. Para qualquer geometria ou solução desta família observamos que o campo escalar não massivo decai com o tempo, e o tipo de decaimento depende do valor da constante $C$. Assim, toda a família de soluções obtida mostrou-se estável. 


\section{Abstract}

We found classes of static and spherically symmetric black hole solutions on the brane for a vacuum configuration, non-null cosmological constant, embedded into a 5dimensional asymptotically anti-de Sitter bulk. In the asymptotically de Sitter case, the only solution obtained was the extreme Schwarzschild-de Sitter geometry. In the asymptotically anti-de Sitter case, a class of solutions was obtained where a particular geometry is fixed by a constant, $C$. We studied the behaviour of these solutions near and far from the horizon. Next, we studied the time evolution of a scalar field outside the black hole. For the effective potential as function of tortoise coordinate, the analytical results near the horizon agree with numerical calculations. The scalar field decays with time and the particular form of decay depends of the value for $C$. Thus, all solutions in the obtained class are stable. 


\section{Agradecimentos}

À minha família: Aluísio, Janine, Juliana e, especialmente, Graça, minha mãe.

Aos meus amigos Sorocabanos: Adriano, André, Bruno, Daniel, Keny, Hidalgo e sua rádio Super. Agradeço-lhes pela grande e duradoura amizade.

Aos meus tios e primos que me hospedaram no início dos meus estudos na USP em 2001.

Aos meus amigos junglists que freqüentam e prestigiam as festas de drum and bass: Eliel e Rafael.

Aos meus amigos de graduação: Adriana, Carol, Carioca, Rafael, Raul, Rubens e Vanks. Agradeço-lhes o companherismo.

Aos meus amigos de pós graduação: Alan, Denny, Jeferson, Rodrigos e Walney. Agradeço-lhes todo tipo de ajuda.

Ao meu orientador, Carlos Molina Mendes, por ter me dado a oportunidade de realizar este trabalho.

À CAPES, pelo apoio financeiro. 
Às pessoas que eu amo. 
O Ser é, o Não-Ser não é.

Parmênides de Eléia 


\section{Sumário}

1 Introdução 1

2 Geometria e Relatividade Geral 4

2.1 Introduzindo a geometria . . . . . . . . . . . . . . 4

2.2 Simetrias em Relatividade Geral . . . . . . . . . . . . . . . . . 8

2.3 Espaços estacionários, estáticos e esfericamente simétricos . . . . . . . 14

2.4 Conexão, curvatura e equações de Einstein . . . . . . . . . . . . 18

3 Universos Brana $\quad 22$

3.1 Dimensões extras . . . . . . . . . . . . . . . . . . . . . . . . 22

3.2 Modelos Randall-Sundrum . . . . . . . . . . . . . . . . . . . . 24

3.3 Projeção das equações de Einstein na 3-brana . . . . . . . . . . . . 28

4 Buracos Negros Estáticos com Simetria Esférica 36

4.1 Horizontes . . . . . . . . . . . . . . . . . . . . . . . . 36

4.2 Extensão da métrica . . . . . . . . . . . . . . . . . . 40

4.3 Construção de soluções . . . . . . . . . . . . . . . . . . . . . . . 42

5 Soluções com constante cosmológica $\quad 45$

5.1 Soluções assintoticamente de Sitter . . . . . . . . . . . . . . 45

$5.1 .1 \quad$ Raízes de $A(r)$ e $g(r) \ldots \ldots \ldots \ldots \ldots . \ldots . \ldots . \ldots 45$ 
5.1 .2 Família de Soluções . . . . . . . . . . . . . . . . 49

5.2 Soluções assintoticamente anti-de Sitter . . . . . . . . . . . . . . . 49

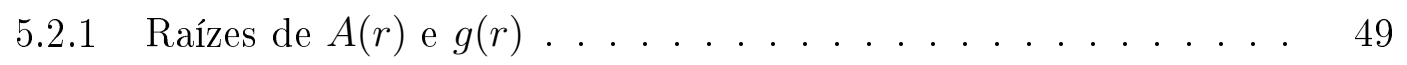

5.2 .2 Família de Soluções . . . . . . . . . . . . . . . . . 51

5.2.3 Comportamento próximo ao horizonte . . . . . . . . . . 57

6 Perturbações Escalares $\quad 61$

6.1 Introduzindo o campo escalar . . . . . . . . . . . . . . . . 61

6.2 Cálculo numérico . . . . . . . . . . . . . . . . . . 68

6.3 Resultados numéricos . . . . . . . . . . . . . . . 70

6.3.1 Decaimento oscilatório-exponencial . . . . . . . . . . . 72

6.3.2 Decaimento exponencial não-oscilatório . . . . . . . . . . . 73

6.3.3 Decaimento em lei de potência . . . . . . . . . . 75

$\begin{array}{lll}7 & \text { Conclusões } & 77\end{array}$ 


\section{Capítulo 1}

\section{Introdução}

As palavras teoria e relatividade, quando juntas, referem-se a uma das idéias mais populares da ciência, embora pouco compreendida pela maioria das pessoas. Quando Albert Einstein publicou sua Teoria da Relatividade em artigos nos anos de 1905 e 1916, não poderia prever o impacto de suas idéias na visão de mundo e na vida das pessoas, muito menos a popularidade de sua teoria. A Teoria da Relatividade modificou as noções de espaço e tempo, tornando-os interdependentes, de tal forma que não podemos tratá-los isoladamente, mas como um espaço-tempo. Ambos os conceitos, a partir de então, não são absolutos como na Mecânica Newtoniana, mas relativos, dependem do observador.

Outra grande contribuição de Einstein foi relacionar geometria com matéria ou energia, isto é, matéria determina a geometria e vice-versa. No entanto, sua equação, proposta em 1916, foi modificada quando a constante cosmológica, $\Lambda_{4 D}$, foi adicionada para que sua teoria estivesse de acordo com a idéia de um universo estático em grande escala. Entretanto, a constante cosmológica foi abandonada quando Edwin Hubble constatou a expansão do universo na década de vinte do século passado e, somente no final do século XX, foi ressuscitada para tentar esclarecer a expansão acelerada do universo.

Em 1916, logo depois da publicação da Teoria da Relatividade Geral, um físico alemão chamado Karl Schwarzschild obteve a solução exata das equações de Einstein no vácuo com constante cosmológica nula para uma geometria esfericamente simétrica. Tal solução ficou conhecida como solução de Schwarzschild. Os elementos da métrica de Schwarzschild, no sistema de coordenadas esférico, divergem para dois valores da 
coordenada radial, $r$ : em $r=0$ e em $r=2 m$. A última divergência pode ser removida, desde que uma mudança para um sistema de coordenadas apropriado seja feita. Como veremos no capítulo 2 , um sistema de coordenadas poderá não ser eficaz para descrever completamente todo um espaço-tempo. A singularidade em $r=0$ é conhecida como singularidade física ou essencial e o valor $2 m$ corresponde ao raio do horizonte de eventos, que é uma superfície de mão única, ou seja, depois de penetrar nesta superfície não há como sair dela, nem mesmo a luz consegue. Por isso, esta solução descreve o buraco negro de Schwarzschild.

Como foi dito acima, a Teoria da Relatividade tranformou dois entes distintos num único só. O espaço-tempo quadridimensional é onde e quando eventos ocorrem. Entretanto, no mesmo século, nos anos vinte, Kaluza e Klein nos trabalhos [1, 2] propuseram a existência de uma dimensão extra compacta na tentativa, sugerida pelo primeiro, de unificar o eletromagnetismo com a gravitação. Podemos pensar em dimensões compactas através do conhecido exemplo da mangueira no jardim. Vista do alto, uma mangueira parece uma linha, um objeto unidimensional, entretanto se chegarmos perto, veremos que este objeto além de comprimento, possui largura e espessura, ou seja, é um objeto tridimensional. Nos anos 90, a teoria que buscava, e ainda busca, unificar as forças da Física é a Teoria de Cordas, através do uso de 6 dimensões espaciais extras compactas (veja por exemplo [3]). Dessa forma, temos um espaço-tempo com 9 dimensões espaciais e 1 dimensão temporal, este espaço-tempo 10-dimensional é chamado de bulk. Atualmente existe a possibilidade, desde que modelos teóricos mostrem-se realistas, de detecção indireta de dimensões extras através de colisões de partículas elementares no LHC (Large Hadronic Collider), como pode ser visto em [4].

A unificação da Física torna-se necessária pois em altos níveis de energia efeitos quânticos não são desprezíveis, o que conduz à unificação das duas teorias mais bem sucedidas do século XX, a Teoria da Relatividade e a Mecânica Quântica. Além disso, a Teoria de Cordas é uma candidata a resolver o problema da hierarquia, que é a diferença entre a escala de massa eletrofaca, $M_{f} \sim 10^{3} \mathrm{GeV}$, e a escala de massa de Planck 4dimensional, $M_{p} \sim 10^{19} \mathrm{GeV}$, ou seja, é a diferença entre as forças eletrofraca e a força gravitacional, sendo $M_{p}=G_{N}^{-1 / 2}$, onde $G_{N}$ é a constante gravitacional Newtoniana. Obter uma teoria de campos que envolva o modelo padrão e a gravitação seria difícil devido a essa diferença de escalas. Uma opção para resolver o problema da hierarquia é o uso de dimensões extras. Neste contexto, modelos de mundo brana surgem como tentativa de resolver este problema com auxílio de dimensões extras. O modelo tipo Randall-Sundrum II, que será usado neste trabalho, consiste de uma brana quadrimen- 
sional imersa num bulk anti-de Sitter 5-dimensional com simetria $\mathbb{Z}_{2}$. Todos os campos, exceto o gravitacional, estão confinados na brana.

Bronnikov et al. mostra em [5] como obter uma ampla classe de soluções que descrevem buracos negros estáticos esfericamente simétricos na brana. A partir da função $A(r)=g_{t t}$ da métrica pode-se obter uma família de funções $B(r)=-1 / g_{r r}$ e, logo em seguida, fixando um valor para uma determinada constante de integração $C$ a solução fica completa. Cada membro da família corresponde a um valor de $C$ e a uma determinada geometria. Neste trabalho, utilizamos os $A(r)^{\prime}$ 's das geometrias Schwarzschild-de Sitter e Schwarzschild anti-de Sitter para obter uma família de funções $B(r)$ numa brana com vácuo e constante cosmológica não nula. Embora para o primeiro caso a única solução obtida tenha sido a já conhecida solução Schwarzschild-de Sitter extrema, para o segundo caso, obtivemos numericamente uma família de soluções na brana. Estudamos as características desta família longe e próxima do horizonte de eventos, determinando, por exemplo, a gravidade superficial.

O passo seguinte foi verificar a estabilidade da família de soluções obtida. Estabilidade das soluções das equações de Einstein é objeto de estudo desde a década de 50 do século passado quando Regge e Wheeler, no trabalho [30], estudaram a solução de Schwarzschild submetida a uma perturbação. Ambos verificaram que a solução de Schwarzschild é sempre estável, ou seja, a perturbação diminui com o tempo. A família de soluções obtida, a partir de um $A(r)$ Schwarzschild anti-de Sitter, mostrou-se estável para o caso de uma perturbação escalar para quaisquer valores de $C$. Todo o cálculo de estabilidade, que envolveu a evolução do campo escalar $\Phi$, foi realizado com auxílio de cálculo numérico, por meio do problema das coordenadas duplas nulas.

Podemos, então, descrever todos os passos contidos neste trabalho da seguinte forma: o capítulo 2 refere-se à geometria diferencial, ou seja, todo instrumental matemático necessário para se ter alguma compreensão da Teoria de Relatividade Geral; o capítulo 3 refere-se aos mundos branas, com destaques aos modelos Randall-Sundrum I e II e à projeção das equações de Einstein na brana; o capítulo 4 refere-se a buracos negros estáticos com simetria esférica, com destaques às definições de horizontes de eventos e Killing e a construção de soluções desse tipo; o capítulo 5 refere-se às famílias de soluções de buracos negros estáticos e esfericamente simétricos na brana obtidas a partir do $A(r)$ Schwarzschild-de Sitter e Schwarzschild anti-de Sitter; o capítulo 6 refere-se à estabilidade da família de soluções obtida no capítulo 5 quando submetida a um campo escalar não massivo; o capítulo 7 refere-se às conclusões finais deste trabalho. 


\section{Capítulo 2}

\section{Geometria e Relatividade Geral}

Teoria Geométrica da Gravitação poderia ser o nome da Teoria da Relatividade Geral tamanha a importância da Geometria Diferencial na sua formulação. Por isso, neste capítulo um pouco de geometria será exposto com intuito de nos fornecer uma base para compreensão dos capítulos subseqüentes. Conceitos como variedade, métrica entre outros serão apresentados.

\subsection{Introduzindo a geometria}

Um conceito fundamental em geometria diferencial é o de variedade. Tal conceito captura a idéia de um espaço que pode ser curvado e ter uma topologia complicada, porém localmente se assemelha ao $\mathbb{R}^{n}$. Para se ter um uma definição mais rigorosa de variedade é necessário ter em mente outros conceitos tais como mapa, difeomorfismo, sistema de coordenadas, atlas e espaços tangentes.

Um mapa, $\phi$, entre dois conjuntos $M$ e $N$ é a relação que assegura para cada elemento de $M$ um elemento em $N$. Tal relação pode ser expressa por $\phi: M \rightarrow N$. Dado dois mapas $\phi: A \rightarrow B$ e $\psi: B \rightarrow C$, podemos definir a composição $\psi \circ \phi: A \rightarrow C$ pela operação $(\psi \circ \phi)(a)=\psi(\phi(a))$. Dessa forma, $a \in A, \phi(a) \in B$ e $(\psi \circ \phi)(a) \in C$. Existem mapas do tipo injetor, sobrejetor e bijetor. Um mapa $\phi$ é injetor se cada elemento de $N$ tem no máximo um elemento de $M$ correspondente. Se o mapa for sobrejetor, cada elemento de $N$ tem no mínimo um elemento de $M$ correspondente. Caso o mapa seja injetor e sobrejetor, ao mesmo tempo, ele se chamará bijetor. Neste caso, podemos definir o mapa inverso $\phi^{-1}: N \rightarrow M$ por $\left(\phi^{-1} \circ \phi\right)(a)=a$. O conjunto $M$ é chamado 
de domínio do mapa $\phi$ e o conjunto de pontos em $N$, que é mapeado por $M$, é chamado imagem de $\phi$.

Uma bola aberta, na topologia induzida pela métrica usual, é o conjunto de todos pontos $x$ no $\mathbb{R}^{n}$ tal que $|x-y|<r$, para algum $y \in \mathbb{R}^{n}$ fixo e $r \in \mathbb{R}$, onde $|x|$ é uma norma usual. Um conjunto aberto no $\mathbb{R}^{n}$ é construído de uma arbitrária união de bolas abertas, em outras palavras, $V \subset \mathbb{R}^{n}$ é aberto se, para qualquer $y \in V$, há uma bola aberta centrada em $y$ que está completamente dentro de $V$. Se um mapa entre conjuntos abertos do $\mathbb{R}^{n}$ é $p$ vezes diferenciável ele é dito $C^{p}$ (está sendo usada a definição usual de diferenciabilidade entre abertos do $\mathbb{R}^{n}$ ). Caso um mapa seja infinitamente diferenciável, será dito liso e indicado por $C^{\infty}$. Dado duas variedades, $M$ e $N$, e os mapas $\phi: M \rightarrow N$ e $\phi^{-1}: N \rightarrow M$ lisos e invertíveis, $M$ e $N$ serão chamados difeomórficos e $\phi$ será chamado de difeomorfismo.

Um sistema de coordenadas consiste de um sub-conjunto $U$ de um conjunto $M$, que está relacionado com o $\mathbb{R}^{n}$ através de um mapa injetor $\phi: U \rightarrow \mathbb{R}^{n}$, tal que a imagem $\phi(U)$ é um aberto no $\mathbb{R}^{n}$. Um atlas é uma coleção de sistemas de coordenadas $\left\{\left(U_{\alpha}\right.\right.$, $\left.\left.\phi_{\alpha}\right)\right\}$ que satisfaz duas condições:

1. A união de $U_{\alpha}$ é igual a $M$, isto é, $U_{\alpha}$ cobre todo $M$.

2. Se dois sistemas de coordenadas se interseccionam, $U_{\alpha} \cap U_{\beta} \neq \emptyset$, logo o mapa $\left(\phi_{\alpha} \circ \phi_{\beta}^{-1}\right)$ leva os pontos de $\phi_{\beta}\left(U_{\alpha} \cap U_{\beta} \subset \mathbb{R}^{n}\right)$ em $\phi_{\alpha}\left(U_{\alpha} \cap U_{\beta} \subset \mathbb{R}^{n}\right)$ e todos esses mapas devem ser lisos onde são definidos.

A importância em se falar de atlas e sistema de coordenadas reside no fato de que muitas variedades não podem ser inteiramente cobertas por um único sistema de coordenadas. Como exemplo, podemos citar o espaço-tempo de Schwarzschild, que não é bem descrito pelas coordenadas $(t, r, \theta, \phi)$ em uma região que inclui o horizonte de eventos.

Introduziremos, logo em seguida, estruturas características de uma variedade: os espaços tangente e cotangente. O espaço tangente $V$, num ponto $P$ de uma variedade $\mathcal{M}$, é formado por operadores que levam funções na variedade a números reais. Isto é, dado um operador derivada direcional $d / d \lambda$, onde $\lambda$ é um parâmetro ao longo de uma 
curva em $\mathcal{M}$, sua aplicação a uma função $f$ definida na variedade pode ser escrita como

$$
\frac{d f}{d \lambda}=\frac{d x^{\mu}}{d \lambda} \partial_{\mu} f
$$

onde $\partial_{\mu}=\partial / \partial x^{\mu}$ é a derivada parcial. A base desse espaço é formada por operadores derivada parcial, $\partial_{\mu}$, que são bases de um espaço vetorial de derivadas direcionais que é identificado como o espaço tangente (veja [15] capítulo 2). A partir de (2.1), vemos que vetores contravariantes $d x^{\mu} / d \lambda=X^{\mu}$ são elementos desse espaço.

O espaço cotangente ou dual $V^{*}$, num ponto $P$ da variedade $\mathcal{M}$, é formado por operadores que levam vetores do espaço tangente a números reais, ou seja, $\omega: V \rightarrow \mathbb{R}$. Tais operadores são 1-formas (covetores) que podem ser indicadas pelo gradiente $d f$ de uma função $f$

$$
d f\left(\frac{d}{d \lambda}\right)=\frac{d f}{d \lambda}
$$

onde $d / d \lambda$ é um vetor tangente a uma curva de parâmetro $\lambda$ na variedade e pode ser escrito com auxílio de (2.1) como

$$
\frac{d}{d \lambda}=\frac{d x^{\mu}}{d \lambda} \partial_{\mu}
$$

e, dessa forma, (2.2) fica

$$
d f=\partial_{\mu} f d x^{\mu}
$$

Podemos, então, interpretar o gradiente das coordenadas, $d x^{\mu}$, como a base do espaço cotangente, pois, por definição, uma base de um espaço tangente, $\hat{e}_{\nu}$, e uma base de um espaço cotangente, $\hat{u}^{\mu}$, devem satisfazer $\hat{u}^{\mu} \hat{e}_{\nu}=\delta_{\nu}^{\mu}$. Esta relação é satisfeita quando $\partial_{\nu}$ e $d x^{\mu}$ são bases dos espaços tangente e cotangente, respectivamente. Ou seja,

$$
d x^{\mu} \partial_{\nu}=\delta_{\nu}^{\mu}
$$

Uma 1-forma pode, por conseqüência, ser escrita como $\omega=\omega_{\mu} d x^{\mu}$ e, além disso, podemos observar que vetores covariantes $\omega_{\mu}$ são elementos desse espaço dual.

Em uma variedade diferenciável $\mathcal{M}$, tensores são definidos de forma natural como aplicações multilineares de vetores e covetores em um ponto de $\mathcal{M}$ até $\mathbb{R}$. O conjunto de aplicações de ordem $(k, l)$ forma o espaço tangente de tensores

$$
T: \underbrace{V^{*} \otimes \cdots \otimes V^{*}}_{k} \otimes \underbrace{V \otimes \cdots \otimes V}_{l} \rightarrow \mathbb{R}
$$


com $k$ índices covariantes e $l$ índices contravariantes. Considerando o conjunto de espaços vetoriais de um dado tipo em cada ponto da variedade, obtém-se um campo vetorial. É importante ressaltar que sem estruturas adicionais não existe relação entre espaços tangentes em pontos distintos. Entre estas estruturas adicionais está derivada covariante, que será discutida na seção 2.4 .

Qualquer tensor covariante simétrico de ordem 2 define uma métrica $g: V \otimes V \rightarrow \mathbb{R}$, ou em termos de suas componentes em um sistema de coordenadas, $g_{\mu \nu}(x)$. Uma variedade equipada com uma métrica é chamada variedade Riemanniana. A métrica fornece uma noção de causalidade e quadricomprimento e é usual representá-la através da distância infinitesimal, $d s$, entre dois pontos $x^{\mu}$ e $x^{\mu}+d x^{\mu}$,

$$
d s^{2}=g_{\mu \nu}(x) d x^{\mu} d x^{\nu} .
$$

A equação (2.7) é conhecida como elemento de linha. O quadrado do comprimento ou norma de um vetor $X^{\mu}$ é definido como

$$
X^{2}=g_{\mu \nu}(x) X^{\mu} X^{\nu},
$$

onde vetores do tipo espaço têm $X^{2}<0$, vetores do tipo tempo têm $X^{2}>0$ e vetores do tipo luz ou nulo têm $X^{2}=0$.

Podemos representar a métrica na sua forma canônica e, dessa forma, suas componentes são indicadas por

$$
g_{\mu \nu}=\operatorname{diag}(+1,+1, \ldots,-1,-1,0, \ldots, 0),
$$

onde diag significa os elementos da diagonal principal da matriz que representa $g_{\mu \nu}$. Se representarmos o número de +1 's por $t$ e o número de -1 's por $s$, a diferença entre $s-t$ nos fornece a assinatura da métrica. Quando uma métrica tem $s=0$ ela é chamada de Euclidiana ou Riemanniana e quando tem $t=1$ ela é dita Lorentzina. Quando diag possui zeros, a métrica será degenerada. Na Relatividade Geral, métricas de interesse são do tipo Lorentziana e não degeneradas.

Uma solução das equações de Einstein, uma métrica, pode não ser bem comportada numa determinada região do espaço-tempo quando escrita num sistema de coordenadas qualquer. Devido ao fato de que uma variedade pode não ser coberta inteiramente por um único sistema de coordenadas, uma mudança para um outro sistema de coordenadas pode ser realizada e, dessa forma, a métrica poderá descrever a região anteriormente não 
descrita de forma adequada. Tal procedimento chama-se extensão analítica da métrica.

\subsection{Simetrias em Relatividade Geral}

Entre os conceitos mais importantes em geometria diferencial estão os conceitos de pullback e pushforward. Tais conceitos nos levarão a definições de isometria e campo de Killing e, a partir daí, às noções de espaços estáticos e estacionários na seção seguinte.

Vamos considerar duas variedades $M$ e $N$, que podem não ter o mesmo número de dimensões, com sistemas de coordenadas $x^{\mu}$ e $y^{\alpha}$, respectivamente. O mapa $\phi: M \rightarrow N$ e a função $f: N \rightarrow \mathbb{R}$ podem ser representados de forma ilustrativa através da figura 2.1:

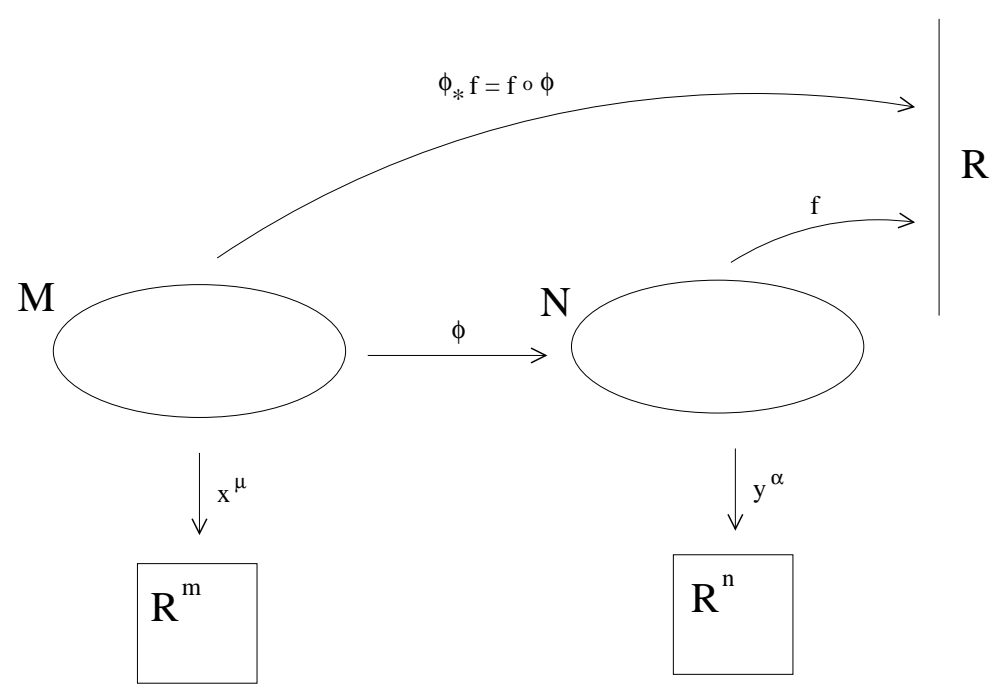

Figura 2.1: Pullback de f por $\phi$ (figura baseada em [15] capítulo 5)

Podemos compor $\phi$ com $f$ e construir o mapa $(f \circ \phi): M \rightarrow \mathbb{R}$, uma função de $M$ em $\mathbb{R}$. Tal operação pode ser indicada por

$$
\phi_{*} f=(f \circ \phi)
$$

e tem o nome de pullback de $f$ por $\phi$. Como o nome sugere, "puxamos" a função $f$ para trás, de $N$ para $M$. Caso tivéssemos uma função em $M, g: M \rightarrow \mathbb{R}$, então não poderíamos fazer o pullback de $g$ por $\phi$, não poderíamos compor $\phi$ com $g$. Isto é 
percebido através do sentido das setas. Dessa forma, não podemos "empurrar" a função $g$ para frente, de $M$ para $N$.

Como vetores podem ser pensados como operadores em espaços vetorias tangentes em cada ponto da variedade e mapeiam funções lisas (funções contínuas e infinitamente diferenciáveis) em números reais, podemos definir o pushforward de um vetor. Sendo $V(p)$ um vetor num ponto $p$ em $M$, o pushforward de $V$ por $\phi$ em um ponto $\phi(p)$ em $N$ será

$$
\left(\phi^{*} V\right)(f)=V\left(\phi_{*} f\right) .
$$

Então o vetor "empurrado" para frente atuando numa função $f$ em $N$ será igual a esse mesmo vetor atuando numa função "puxada" para trás em $M$.

Bases para um espaço vetorial tangente num dado ponto das variedades $M$ e $N$ são dadas por operadores derivadas parciais, como já foi mencionado. Num ponto da variedade $M$, temos um espaço vetorial cuja base será $\partial_{\mu}=\frac{\partial}{\partial x^{\mu}}$ e num ponto da variedade $N$ temos outro espaço vetorial cuja base será $\partial_{\alpha}=\frac{\partial}{\partial x^{\alpha}}$. Podemos relacionar as componentes de $V=V^{\mu} \partial_{\mu}$ em $M$ com as componentes de $\left(\phi^{*} V\right)^{\alpha} \partial_{\alpha}$ em $N$ aplicando a operação de pushforward a uma função lisa com auxílio da regra da cadeia

$$
\begin{aligned}
\left(\phi^{*} V\right)^{\alpha} \partial_{\alpha} f & =V^{\mu} \partial_{\mu}\left(\phi_{*} f\right), \\
& =V^{\mu} \partial_{\mu}(f \circ \phi), \\
& =V^{\mu} \frac{\partial y^{\alpha}}{\partial x^{\mu}} \partial_{\alpha} f .
\end{aligned}
$$

Escrita desta forma, a operação de pushforward lembra uma operação de mudança de coordenadas, pois em (2.12) $\partial y^{\alpha} / \partial x^{\mu}$ faz o papel de uma matriz de mudança de coordenadas. Entretanto, tal operação será uma generalização para mudança de coordenadas apenas quando $M$ e $N$ são a mesma variedade.

Não podemos usar a operação de pullback para vetores, podemos usá-la para 1forma, que são vetores duais. Como uma 1-forma é um mapa linear de vetores a números reais, o pullback de uma 1-forma $\omega$ em $N, \phi_{*} \omega$, pode ser definido por sua ação em um vetor $V$ em $M$ igual à ação de $\omega$ num vetor onde a operação de pushforward foi realizada

$$
\left(\phi_{*} \omega\right)(V)=\omega\left(\phi^{*} V\right) .
$$

Novamente, usando a regra da cadeia, a operação de pullback em formas lembra uma operação de mudança de coordenadas. 
Um tensor $T_{\alpha_{1} \ldots \alpha_{l}}$ covariante em $N$ é uma aplicação linear do produto direto de $l$ covetores ou 1-formas em $\mathbb{R}$. Assim, podemos usar a operação de pullback não somente em formas mas também em tensores covariantes arbitrários. Então, a definição da operação de pullback em tensores covariantes é sua ação em vetores que sofreram a operação de pushforward

$$
\left(\phi_{*} T\right)\left(V^{(1)}, V^{(2)}, \ldots, V^{(l)}\right)=T\left(\phi^{*} V^{(1)}, \phi^{*} V^{(2)}, \ldots, \phi^{*} V^{(l)}\right) .
$$

Da mesma forma, um tensor contravariante $S^{\mu_{1} \ldots \mu_{k}}$ pode sofrer a operação de pushforward

$$
\left(\phi^{*} S\right)\left(\omega^{(1)}, \omega^{(2)}, \ldots, \omega^{(k)}\right)=S\left(\phi_{*} \omega^{(1)}, \phi_{*} \omega^{(2)}, \ldots, \phi_{*} \omega^{(k)}\right) .
$$

As representações das matrizes das operações pullback e pushforward para tensores de ordem elevada podem ser escritas como

$$
\left(\phi_{*} T\right)_{\mu_{1} \ldots \mu_{l}}=\frac{\partial y^{\alpha_{1}}}{\partial x^{\mu_{1}}} \ldots \frac{\partial y^{\alpha_{l}}}{\partial x^{\mu_{l}}} T_{\alpha_{1} \ldots \alpha_{l}}
$$

e

$$
\left(\phi^{*} S\right)^{\alpha_{1} \ldots \alpha_{k}}=\frac{\partial y^{\alpha_{1}}}{\partial x^{\mu_{1}}} \ldots \frac{\partial y^{\alpha_{k}}}{\partial x^{\mu_{k}}} S^{\mu_{1} \ldots \mu_{k}},
$$

para um tensor $T$ de ordem $(0, l)$ e um tensor $S$ de ordem $(k, 0)$. Tensores mistos podem ser "puxados" e "empurrados", desde que $\phi$ seja um difeomorfismo, isto é, seja liso e possua uma inversa, $\phi^{-1}$, igualmente lisa. Como um exemplo, para um tensor $T^{\mu_{1} \ldots \mu_{k}}{ }_{\nu_{1} \ldots \nu_{l}}$ em $M$, a operação de pushforward para pode ser representada por

$$
\left(\phi^{*} T\right)\left(\omega^{(1)}, \ldots, \omega^{(k)}, V^{(1)}, \ldots, V^{(l)}\right)=T\left(\phi_{*} \omega^{(1)}, \ldots, \phi_{*} \omega^{(k)},\left[\phi^{-1}\right]^{*} V^{(1)}, \ldots,\left[\phi^{-1}\right]^{*} V^{(l)}\right),
$$

onde $\omega^{(i)}, s$ são 1-formas em $N$ e os $V^{(i)} s$ são vetores em $N$. Podemos representá-la em componentes da seguinte forma

$$
\left(\phi^{*} T\right)_{\beta_{1} \ldots \beta_{l}}^{\alpha_{1} \ldots \alpha_{k}}=\frac{\partial y^{\alpha_{1}}}{\partial x^{\mu_{1}}} \ldots \frac{\partial y^{\alpha_{k}}}{\partial x^{\mu_{k}}} \frac{\partial x^{\nu_{1}}}{\partial y^{\beta_{1}}} \ldots \frac{\partial x^{\nu_{l}}}{\partial y^{\beta_{l}}} T_{\nu_{1} \ldots \nu_{l}}^{\mu_{1} \ldots \mu_{k}} .
$$

O pullback de um tensor misto $T^{\mu_{1} \ldots \mu_{k}} \nu_{\nu_{1} \ldots \nu_{l}}$ em $N$ pode ser representado por

$$
\left(\phi_{*} T\right)\left(\omega^{(1)}, \ldots, \omega^{(k)}, V^{(1)}, \ldots, V^{(l)}\right)=T\left(\left[\phi^{-1}\right]_{*} \omega^{(1)}, \ldots,\left[\phi^{-1}\right]_{*} \omega^{(k)}, \phi^{*} V^{(1)}, \ldots, \phi^{*} V^{(l)}\right),
$$

onde os $\omega^{(i),} s$ são 1-formas em $M$ e os $V^{(i),} s$ são vetores em $M$. Podemos representá-la 
em componentes da seguinte forma

$$
\left(\phi_{*} T\right)_{\beta_{1} \ldots \beta_{l}}^{\alpha_{1} \ldots \alpha_{k}}=\frac{\partial x^{\nu_{1}}}{\partial y^{\beta_{1}}} \cdots \frac{\partial x^{\nu_{l}}}{\partial y^{\beta_{l}}} \frac{\partial y^{\alpha_{1}}}{\partial x^{\mu_{1}}} \cdots \frac{\partial y^{\alpha_{k}}}{\partial x^{\mu_{k}}} T_{\nu_{1} \ldots \nu_{l}}^{\mu_{1} \ldots \mu_{k}}
$$

Como já foi dito, se representarmos o pullback e o pushforward em componentes, obteremos matrizes de transformação de coordenadas quando $M$ e $N$ são a mesma variedade. Quando $\phi$ é um difeomorfismo e as variedades $M$ e $N$ são a mesma, obtemos um tipo de transformação de coordenadas que é chamada de transformação "ativa". A transformação de coordenadas tradicional é chamada de "passiva". Vamos considerar, como exemplo, uma variedade $n$-dimensional $M$ com funções de coordenadas $x^{\mu}: M \rightarrow \mathbb{R}^{n}$. Podemos mudar de coordenadas introduzindo novas funções de coordenadas $y^{\mu}: M \rightarrow \mathbb{R}^{n}$, mantendo a variedade fixa. Outra forma de realizar essa mudança de coordenadas é usar um difeomorfismo $\phi: M \rightarrow M$ obtendo as novas coordenadas através de pullbacks e pushforwards $\left(\phi_{*} x\right)^{\mu}: M \rightarrow \mathbb{R}^{n}$, movendo os pontos na variedade e depois calculando suas coordenadas.

Um difeomorfismo permite-nos fazer operações de pullback e pushforward em tensores. Podemos, dessa forma, comparar valores de um campo tensorial em diferentes pontos na variedade como, por exemplo, um campo $T^{\mu_{1} \ldots \mu_{k}}{ }_{\nu_{1} \ldots \nu_{l}}(x)$ calculado num ponto $p$ e num ponto $\phi(p)$, onde a operação de pullback foi aplicada, $\phi_{*}\left[T^{\mu_{1} \ldots \mu_{k}}{ }_{\nu_{1} \ldots \nu_{l}}(\phi(p))\right]$. Assim, um outro tipo de derivada é obtido, uma derivada que mostra como campos tensorias mudam sobre um determinado difeomorfismo. Entretanto, precisamos não apenas de um difeomorfismo, mas de uma família de difeomorfismos de um parâmetro, $\phi_{t}$. Tal família pode ser pensada como um mapa liso $\mathbb{R} \times M \rightarrow M$, de tal forma que para cada $t \in \mathbb{R}, \phi_{t}$ é um difeomorfismo e $\phi_{s} \circ \phi_{t}=\phi_{s+t}$, sendo que $\phi_{0}$ é a identidade.

Famílias de difeomorfismo de um parâmetro podem ser pensadas como originárias de campos vetoriais e vice-versa. Em cada ponto na variedade, sob uma família de $\phi_{t}$, temos curvas. Essas curvas cobrem toda variedade e, dessa forma, podemos definir campos vetorias, $V(x)$, que são o conjunto de vetores tangentes a essas curvas em cada ponto, calculados em $t=0$. De forma contrária, podemos definir uma famíla de difeomorfimos de um parâmetro através de campos vetoriais. Dado um campo vetorial, $V^{\mu}(x)$, podemos definir as curvas integrais, $x^{\mu}(t)$, como sendo as curvas que resolvem a equação

$$
\frac{d x^{\mu}}{d t}=V^{\mu}(x)
$$

Soluções para (2.21) em variedades são garantidas por teoremas que podem ser vistos, por exemplo, em [18] capítulo 4. 
Podemos obter uma famíla de difeomorfismos parametrizada por $t$, a partir de um campo vetorial $V^{\mu}(x)$, e calcular a rapidez com que tensores variam conforme percorrem curvas integrais, que são soluções de (2.21). Para um $t$ qualquer, podemos definir a variação de um tensor por

$$
\Delta_{t} T_{\nu_{1} \ldots \nu_{l}}^{\mu_{1} \ldots \mu_{k}}(p)=\phi_{t *}\left[T_{\nu_{1} \ldots \nu_{l}}^{\mu_{1} \ldots \mu_{k}}\left(\phi_{t}(p)\right)\right]-T_{\nu_{1} \ldots \nu_{l}}^{\mu_{1} \ldots \mu_{k}}(p)
$$

Podemos representar esta variação através da figura 2.2:

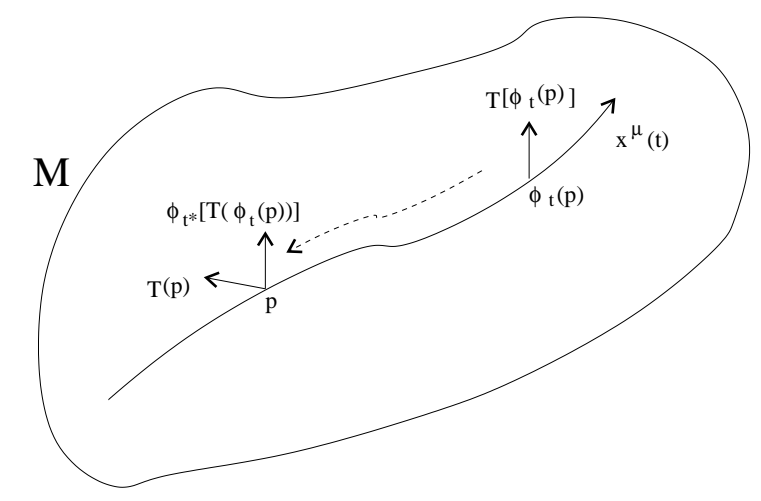

Figura 2.2: Variação de um tensor $T_{\nu_{1} \ldots \nu_{l}}^{\mu_{1} \ldots \mu_{k}}$ (figura baseada em [15] capítulo 5)

Dessa forma, podemos definir a derivada de um tensor ao longo de um campo vetorial. Tal operação chama-se derivada de Lie

$$
\mathcal{L}_{V} T^{\mu_{1} \ldots \mu_{k}}{ }_{\nu_{1} \ldots \nu_{l}}=\lim _{t \rightarrow 0}\left(\frac{\Delta_{t} T^{\mu_{1} \ldots \mu_{k}} \nu_{1} \ldots \nu_{l}}{t}\right)
$$

A derivada de Lie é independente do sistema de coordenadas, é uma mapa de um tensor do tipo $(k, l)$ a um tensor do tipo $(k, l)$, é linear $(\nabla(T+S)=\nabla T+\nabla S$, onde $T$ e $S$ são tensores) e obedece a regra de Leibniz $(\nabla(T \otimes S)=(\nabla T) \otimes S+T \otimes(\nabla S))$.

Para facilitar a discussão sobre a derivada de Lie e outras operações relativas à ela, vamos adotar um sistema de coordenadas $x^{\mu}$ em que $x^{1}$ é um parâmetro ao longo das curvas integrais. Assim, o campo vetorial toma a forma $V=\frac{\partial}{\partial x^{1}}$, ou seja, o campo tem as componentes $V^{\mu}=(1,0,0, \ldots, 0)$ e um difeomorfismo de uma família de um parâmetro, $\phi_{t}$, produz a transformação de coordenadas de $x^{\mu}$ a $y^{\mu}=\left(x^{1}+t, x^{2}, \ldots, x^{n}\right)$. Conseqüentemente, a matriz de pullback fica

$$
\frac{\partial y^{\nu}}{\partial x^{\mu}}=\delta_{\mu}^{\nu},
$$


e as componentes do tensor que sofreu a operação de pullback de $\phi_{t}(p)$ a $p$ ficam

$$
\phi_{t *}\left[T_{\nu_{1} \ldots \nu_{l}}^{\mu_{1} \ldots \mu_{k}}\left(\phi_{t}(p)\right)\right]=T_{\nu_{1} \ldots \nu_{l}}^{\mu_{1} \ldots \mu_{k}}\left(x^{1}+t, x^{2}, \ldots, x^{n}\right) .
$$

Nesse sistema de coordenadas, a derivada de Lie de um campo tensorial é expressa por

$$
\mathcal{L}_{V} T_{\nu_{1} \ldots \nu_{l}}^{\mu_{1} \ldots \mu_{k}}=\frac{\partial}{\partial x^{1}} T_{\nu_{1} \ldots \nu_{l}}^{\mu_{1} \ldots \mu_{k}}
$$

ou para um campo vetorial $U^{\mu}(x)$

$$
\mathcal{L}_{V} U^{\mu}=\frac{\partial U^{\mu}}{\partial x^{1}} .
$$

A derivada de Lie pode também ser expressa por um comutador

$$
\mathcal{L}_{V} U^{\mu}=[V, U]^{\mu},
$$

sendo este comutador dado por

$$
[V, U]^{\mu}=V^{\nu} \partial_{\nu} U^{\mu}-U^{\nu} \partial_{\nu} V^{\mu}
$$

Vamos fazer o uso de (2.28), com o auxílio de (2.29), para o caso da métrica $g_{\mu \nu}$. Obtemos, então,

$$
\begin{aligned}
\mathcal{L}_{V} g_{\mu \nu} & =V^{\sigma} \nabla_{\sigma} g_{\mu \nu}+\left(\nabla_{\mu} V^{\lambda}\right) g_{\lambda \nu}+\left(\nabla_{\nu} V^{\lambda}\right) g_{\mu \lambda} \\
& =\nabla_{\mu} V_{\nu}+\nabla_{\nu} V_{\mu} \\
& =2 \nabla_{(\mu} V_{\nu)} .
\end{aligned}
$$

onde $\nabla_{\mu}$ é a derivada covariante de $g_{\mu \nu}$.

Dizemos que um difeomorfismo, $\phi$, é uma simetria de algum tensor, $T$, se depois que aplicamos a operação de pullback neste tensor ele permanece o mesmo, ou seja,

$$
\phi_{*} T=T \text {. }
$$

Se uma famíla de simetrias de um parâmetro, $\phi_{t}$, é gerada por um campo vetorial $V^{\mu}(x)$, por (2.27) usando (2.31) temos

$$
\mathcal{L}_{V} T=0 .
$$

Uma conseqüência desta simetria é o fato de que o tensor $T$ é simétrico sob uma família 
de difeomorfismos de um parâmetro e podemos sempre obter um sistema de coordenadas onde as componentes de $T$ são sempre independentes de uma coordenada

$$
\mathcal{L}_{V} T=\frac{\partial T}{\partial x^{1}}=0 .
$$

As componentes de $T$ não dependem de $x^{1}$.

Uma importante simetria é aquela relacionada à métrica, ou seja, $\phi_{*} g_{\mu \nu}=g_{\mu \nu}$. Um difeomorfismo que gera tal simetria é chamado de isometria. Se uma família de isometrias de um parâmetro é gerada por um campo vetorial $V^{\mu}(x)$, este campo é do tipo Killing. A condição que $V^{\mu}$ seja um campo de Killing é a seguinte:

$$
\mathcal{L}_{V} g_{\mu \nu}=0,
$$

ou usando (2.30)

$$
\nabla_{(\mu} V_{\nu)}=0 .
$$

A equação (2.35) é conhecida como equação de Killing. Então, se um espaço-tempo tem um campo de Killing, podemos escrever a métrica que o representa independente de uma coordenada.

\subsection{Espaços estacionários, estáticos e esfericamente simétricos}

Para espaços com dimensão $n \geq 2$, podemos ter mais campos de Killing do que dimensões. Isto se deve ao fato que um conjunto de campos de Killing pode ser line-

armente independente, mesmo que num dado ponto da variedade os vetores de Killing sejam linearmente dependentes, e, dessa forma, pode gerar um outro campo de Killing (desde que a combinação linear seja a coeficientes constantes).

Um espaço de dimensão $n$ tem simetria máxima quando tem o número de campos de Killing linearmente independentes associados a ele dado por: $n(n+1) / 2$. Por exemplo, no $\mathbb{R}^{n}$ temos duas classes de isometrias de um parâmetro: translações e rotações. Há $n$ translações, uma para cada direção, e $n(n-1)$ rotações (para cada direção há $n-1$ direções que podemos rodar, e para evitar uma contagem repetida dividimos por 2, ou seja, fazer uma rotação em torno de $z$ no sentido horário e anti-horário é a mesma 
coisa). Assim temos

$$
n+\frac{n(n-1)}{2}=\frac{n(n+1)}{2}
$$

campos de Killing no $\mathbb{R}^{n}$. Como exemplo de geometrias com simetria máxima temos o espaço-tempo de Minkowski, cuja métrica é dada por

$$
d s^{2}=d t^{2}-d x^{2}-d y^{2}-d z^{2},
$$

onde $t$ é a coordenada temporal e $(x, y, z)$ são coordenadas cartesianas. Outros casos importantes são o espaço-tempo de Sitter (dS), cuja métrica é dada por

$$
d s^{2}=\left(1-\frac{r^{2}}{a^{2}}\right) d t^{2}-\left(1-\frac{r^{2}}{a^{2}}\right)^{-1} d r^{2}-r^{2}\left(d \theta^{2}+\operatorname{sen}^{2} d \phi^{2}\right),
$$

e o espaço-tempo anti-de Sitter (AdS), cuja métrica é

$$
d s^{2}=\left(1+\frac{r^{2}}{a^{2}}\right) d t^{2}-\left(1+\frac{r^{2}}{a^{2}}\right)^{-1} d r^{2}-r^{2}\left(d \theta^{2}+\operatorname{sen}^{2} d \phi^{2}\right),
$$

sendo $t$ a coordenada temporal, $r$ a coordenada radial, $\theta$ e $\phi$ são as coordenadas angulares. A constante $a$ é positiva e está relacionada com a constante cosmológica por

$$
a^{2}= \pm \frac{3}{\Lambda_{4 D}},
$$

onde $\Lambda_{4 D}>0$ no caso de Sitter e $\Lambda_{4 D}<0$ no caso anti-de Sitter.

Um espaço-tempo que possui simetria esférica têm campos de Killing linearmente independentes que satisfazem as relações de comutação abaixo:

$$
\begin{aligned}
& {\left[V^{(1)}, V^{(2)}\right]=V^{(3)},} \\
& {\left[V^{(2)}, V^{(3)}\right]=V^{(1)}}
\end{aligned}
$$

e

$$
\left[V^{(3)}, V^{(1)}\right]=V^{(2)}
$$

Como exemplo, podemos citar o espaço-tempo de Schwarzschild, que possui a métrica

$$
d s^{2}=\left(1-\frac{2 m}{r}\right) d t^{2}-\left(1-\frac{2 m}{r}\right)^{-1} d r^{2}-r^{2}\left(d \theta^{2}+\operatorname{sen}^{2} \theta d \phi^{2}\right),
$$

onde $m$ é uma constante real positiva, que pode ser interpretada como a massa. 
Um espaço-tempo é dito estácionário se ele admite um campo de Killing do tipo tempo [14]. Portanto, uma métrica é estacionária se existe um sistema de coordenadas onde ela é independente do tempo, isto é,

$$
\frac{\partial g_{\mu \nu}}{\partial x^{0}}=0,
$$

sendo $x^{0}$ é uma coordenada do tipo tempo. Se definirmos um campo vetorial $X^{\mu}=\delta_{0}^{\mu}$, logo a derivada de Lie da métrica será

$$
\begin{gathered}
\mathcal{L}_{X} g_{\mu \nu}=X^{\sigma} \nabla_{\sigma} g_{\mu \nu}+\left(\nabla_{\mu} X^{\lambda}\right) g_{\lambda \nu}+\left(\nabla_{\nu} X\right) g_{\mu \lambda}, \\
\mathcal{L}_{X} g_{\mu \nu}=\delta_{0}^{\sigma} \nabla_{\sigma} g_{\mu \nu}=0,
\end{gathered}
$$

por (2.43). Como $\mathcal{L}_{X} g_{\mu \nu}$ é um tensor, então ele será zero em qualquer sistema de coordenadas e $X^{\mu}$ é um campo de Killing, de acordo com (2.34), do tipo tempo. De forma inversa, dado um campo de Killing do tipo tempo $X^{\mu}$, sempre existe um sistema de coordenadas que satisfaz (2.44).

Um espaço-tempo é dito estático se ele pode ser folheado por uma família de hipersuperfícies ortogonais a um campo de Killing do tipo tempo, de acordo com [14]. Num espaço-tempo estático, há um sistema de coordenadas adaptado ao campo de Killing do tipo tempo de tal forma que a métrica é independente da coordenada temporal e não há termos cruzados, isto é, $g_{0 a}=0$. Portanto, para falarmos de espaço-tempo estático, precisamos do conceito de campo vetorial ortogonal a uma hipersuperfície (uma hipersuperfície em uma variedade de dimensão $n$ é simplesmente uma subvariedade de dimensão $n-1)$. A equação de uma família de hipersuperfícies é dada por

$$
f\left(x^{\mu}\right)=a,
$$

onde diferentes valores de $a$ correspondem a diferentes hipersuperfícies. Dado dois pontos $P$ e $Q$ com coordenadas $x^{\mu}$ e $\left(x^{\mu}+d x^{\mu}\right)$, respectivamente, na mesma hipersuperfície $S$, por (2.45) podemos escrever em primeira ordem de aproximação em torno do ponto $P$

$$
f\left(x^{\mu}+d x^{\mu}\right)=f\left(x^{\mu}\right)+\frac{\partial f}{\partial x^{\mu}} d x^{\mu} .
$$

Entretanto, $f\left(x^{\mu}+d x^{\mu}\right)=f\left(x^{\mu}\right)$ pois os pontos $P$ e $Q$ estão na mesma hipersuperfície. Então, o segundo termo de (2.46) é nulo no ponto $P$. 
Se definirmos um campo vetorial covariante $\eta_{\mu}$ como

$$
\eta_{\mu}=\frac{\partial f}{\partial x^{\mu}},
$$

$\log 0$

$$
\eta_{\mu} d x^{\mu}=g_{\mu \nu} \eta^{\mu} d x^{\nu}=0
$$

no ponto $P$ e nos diz que $\eta_{\mu}$ é ortogonal à $d x^{\mu}$. Consequentemente, $\eta_{\mu}$ é ortogonal à hipersuperfície $S$ pois $d x^{\mu}$ está em $S$ e é chamado de campo vetorial normal à $S$ em $P$. Qualquer outro campo vetorial $X^{\mu}$ ortogonal à $S$ será proporcional à $\eta_{\mu}$, isto é,

$$
X^{\mu}=\lambda(x) \eta^{\mu}
$$

sendo $\lambda(x)$ um fator que depende do ponto onde calculamos. Pode-se mostrar que $\lambda(x)=X^{2}=X^{\mu} X_{\mu}$ (veja [14] capítulo 14) e assim (2.49) fica

$$
X_{\mu}=X^{2} \frac{\partial f}{\partial x^{\mu}} .
$$

Uma métrica estática não contém termos cruzados que envolvem a coordenada do tipo tempo, isto é, uma solução estática não contém termos do tipo $d x^{0} d x^{\mu}$. Para entender isso, considere o intervalo entre os eventos $\left(x^{0}, x^{1}, x^{2}, x^{3}\right)$ e $\left(x^{0}+d x^{0}, x^{1}+\right.$ $\left.d x^{1}, x^{2}, x^{3}\right)$ num dado sistema de coordenadas. Dessa forma, $d s^{2}$ fica

$$
d s^{2}=g_{00}\left(d x^{0}\right)^{2}+2 g_{01} d x^{0} d x^{1}+g_{11}\left(d x^{1}\right)^{2} .
$$

Sob uma reversão temporal $x^{0} \rightarrow x^{0}=-x^{0},(2.51)$ fica

$$
d s^{2}=g_{00}\left(d x^{0}\right)^{2}-2 g_{01} d x^{0} d x^{1}+g_{11}\left(d x^{1}\right)^{2} .
$$

Como numa solução estática $d s^{2}$ é invariante sob uma reversão temporal, $g_{01}$ deve ser zero para que (2.51) e (2.52) coincidam. Da mesma forma, se tivéssemos usado $x^{2}+d x^{2}$ ou $x^{3}+d x^{3}, g_{02}$ ou $g_{03}$ deveriam se anular igualmente.

Dado um espaço-tempo estacionário e um campo de Killing do tipo tempo, isto é, $X^{\mu}=\delta_{0}^{\mu}, \log \mathrm{,}$

$$
X_{\mu}=g_{\mu \nu} X^{\nu}=g_{\mu \nu} \delta_{0}^{\nu}=g_{0 \mu}
$$

e

$$
X^{2}=X_{\mu} X^{\mu}=g_{0 \mu} \delta_{0}^{\mu}=g_{00} .
$$


Então (2.50) fornece com auxílio de (2.53) e (2.54)

$$
g_{0 \mu}=g_{00} \frac{\partial f}{\partial x^{\mu}}
$$

para algum campo escalar $f$. Quando $\mu=0$, (2.55) fica $\partial f / \partial x^{0}=1$ e depois de integrá-la obtemos

$$
f=x^{0}+h\left(x^{a}\right)
$$

onde $h$ é somente uma função das coordenadas do tipo espaço e o índice $a$ vai de 1 a 3 . Agora, vamos fazer uma transformação de coordenadas

$$
x^{0} \rightarrow x^{\prime 0}=x^{0}+h\left(x^{a}\right), \quad x^{a} \rightarrow x^{\prime a}=x^{a} .
$$

Como no caso estacionário, a métrica não depende da coordenada do tipo tempo

$$
\frac{\partial g_{\mu \nu}^{\prime}}{\partial x^{\prime 0}}=0
$$

Nesse sistema de coordenadas, $X^{\prime \mu}=\delta_{0}^{\mu}$ e com o auxílio de (2.55) e (2.56) obtemos

$$
g_{0 \mu}^{\prime}=g_{00}^{\prime} \frac{\partial x^{\prime 0}}{\partial x^{\prime \mu}}
$$

Para o caso $\mu=0$ temos

$$
g_{00}^{\prime}=g_{00} .
$$

e para o caso $\mu \neq 0$ conseguimos

$$
g_{0 a}^{\prime}=0
$$

isto é, não há termos cruzados do tipo $d x^{0} d x^{a}$ e a solução, dessa forma, é estática.

\subsection{Conexão, curvatura e equações de Einstein}

Nas seções anteriores foram introduzidas uma variedade diferenciável e uma métrica Lorentziana como estruturas básicas para descrever o espaço-tempo. Na variedade são definidas de maneira natural funções, curvas parametrizadas, espaços tangentes, vetores e tensores.

Pontos distintos em $\mathcal{M}$ não compartilham, em geral, do mesmo espaço vetorial tangente, exceto o $\mathbb{R}^{n}$, que tem para cada ponto um espaço tangente associado a ele 
igual ao próprio $\mathbb{R}^{n}$. Dessa forma, o operador derivação do $\mathbb{R}^{n}$ não pode ser o operador de derivação de uma variedade qualquer. Para uma variedade qualquer, queremos um operador $\nabla_{\gamma}$ que seja independe do sistemas de coordenadas, seja linear em seus parâmetros, obedeça a regra do produto de Leibniz e se transforme como um tensor. O operador derivada covariante aplicado a um tensor contravariante, $X^{\alpha}$, numa dada direção $\gamma$, é dado por

$$
\nabla_{\gamma} X^{\alpha}=\partial_{\gamma} X^{\alpha}+\Gamma_{\beta \gamma}^{\alpha} X^{\beta},
$$

e para o caso de um tensor covariante, $X_{\alpha}$, é dado por

$$
\nabla_{\gamma} X_{\alpha}=\partial_{\gamma} X_{\alpha}-\Gamma_{\alpha \gamma}^{\beta} X_{\beta}
$$

Se exigirmos que $\nabla_{\gamma} X^{\alpha}$ seja um tensor do tipo $(1,1), \Gamma_{\beta \gamma}^{\alpha}$ deve se transformar de acordo com

$$
\Gamma_{\beta \gamma}^{\alpha}=\frac{\partial x^{\prime \alpha}}{\partial x^{\delta}} \frac{\partial x^{\rho}}{\partial x^{\prime \beta}} \frac{\partial x^{\sigma}}{\partial x^{\prime \gamma}} \Gamma_{\rho \sigma}^{\delta}-\frac{\partial x^{\delta}}{\partial x^{\prime \beta}} \frac{\partial x^{\rho}}{\partial x^{\prime \gamma}} \frac{\partial^{2} x^{\prime \alpha}}{\partial x^{\delta} \partial x^{\rho}} .
$$

Se o segundo termo do lado direito de (2.63) fosse zero, teríamos a usual lei de transformação de tensores do tipo $(1,2)$. Portanto, a presença do segundo termo do lado direito revela que a lei de transformação é inomogênea, ou seja, $\Gamma_{\beta \gamma}^{\alpha}$ não é um tensor. Qualquer objeto que se transforme de acordo com (2.63) é chamado de conexão afim. Uma variedade com uma conexão afim contínua é dita variedade afim. A equação (2.61) num outro sistema de coordenadas pode ser escrita como

$$
\nabla_{\gamma}^{\prime} X^{\prime \alpha}=\partial_{\gamma}^{\prime} X^{\prime \alpha}+\Gamma_{\beta \gamma}^{\alpha} X^{\prime \beta},
$$

sendo

$$
\begin{aligned}
\partial_{\gamma}^{\prime} X^{\prime \alpha} & =\partial_{\gamma}\left(\frac{\partial x^{\prime \alpha}}{\partial x^{\beta}} X^{\beta}\right) \\
& =\frac{\partial x^{\delta}}{\partial x^{\prime \gamma}} \frac{\partial}{\partial x^{\delta}}\left(\frac{\partial x^{\prime \alpha}}{\partial x^{\beta}} X^{\beta}\right) \\
& =\frac{\partial x^{\prime \alpha}}{\partial x^{\beta}} \frac{\partial x^{\delta}}{\partial x^{\prime \gamma}} \partial_{\delta} X^{\beta}+\frac{\partial x^{\delta}}{\partial x^{\prime \gamma}} \frac{\partial^{2} x^{\prime \alpha}}{\partial x^{\beta} \partial x^{\delta}} X^{\beta}
\end{aligned}
$$

e

$$
X^{\prime \beta}=\frac{\partial x^{\prime \beta}}{\partial x^{\rho}} X^{\rho} .
$$

O segundo termo de (2.65) faz com que $\partial_{\gamma}^{\prime} X^{\prime \alpha}$ não seja um tensor. Substituindo (2.65), (2.63) e (2.66) na equação (2.64), com uma troca apropriada de índices mudos veremos que o segundo termo de (2.63) é cancelado com o segundo termo (2.65), fazendo com 
que o operador derivada covariante transforme-se como um tensor.

Uma conexão em que a derivada covariante da métrica é zero é chamada de "compatível com a métrica". Existem infinitas conexões deste tipo. Porém, existe uma única conexão compatível com a métrica e com torção nula. Esta é a conexão usada na relatividade geral, denominada conexão de Christoffel

$$
\Gamma_{\beta \gamma}^{\alpha}=\frac{1}{2} g^{\alpha \delta}\left(\partial_{\beta} g_{\delta \gamma}+\partial_{\gamma} g_{\delta \beta}-\partial_{\delta} g_{\beta \gamma}\right) .
$$

Introduzida a conexão, podemos definir a curvatura. Para a conexão de Christoffel, o tensor curvatura ou tensor de Riemann é dado por

$$
R_{\beta \gamma \delta}^{\alpha}=\partial_{\gamma} \Gamma_{\beta \delta}^{\alpha}-\partial_{\delta} \Gamma_{\beta \gamma}^{\alpha}+\Gamma_{\beta \delta}^{\rho} \Gamma_{\rho \gamma}^{\alpha}-\Gamma_{\beta \gamma}^{\rho} \Gamma_{\rho \delta}^{\alpha} .
$$

Com o uso de contrações do tensor de curvatura, define-se várias quantidades importantes: o tensor de Ricci

$$
R_{\mu \nu}=g^{\gamma \delta} R_{\delta \mu \gamma \nu},
$$

e o escalar de curvatura ou escalar de Ricci

$$
R=g^{\mu \nu} g^{\gamma \delta} R_{\delta \mu \gamma \nu} .
$$

A introdução de matéria é fundamental na descrição da gravidade. Na relatividade geral, isso é feito (em geral) através de campos definidos na variedade, associados a um tensor de energia-momento, $T_{\mu \nu}$. Estes campos obedecem a equações de movimento que em geral podem ser expressas em termos de relações tensoriais. Nestas equações, as derivadas espaciais são derivadas covariantes com relação à conexão definida pela métrica.

Em 1916, Einstein propôs como equação de movimento para as componentes da métrica a relação

$$
G_{\mu \nu}=R_{\mu \nu}-\frac{1}{2} g_{\mu \nu} R=\frac{8 \pi G_{N}}{c^{4}} T_{\mu \nu} .
$$

A equação tensorial acima, um sistema de equações não lineares para as 10 componentes independentes da métrica, é conhecida como equação de Einstein e foi modificada pelo próprio Einstein quando a constante cosmológica, $\Lambda_{4 D}$, foi adicionada para que sua teoria estivesse de acordo com a idéia de um universo estático em grande escala. Então, 
a equação tensorial tornou-se

$$
G_{\mu \nu}+\Lambda_{4 D} g_{\mu \nu}=\frac{8 \pi G_{N}}{c^{4}} T_{\mu \nu} .
$$

A constante $8 \pi G_{N} / c^{4}$ é escolhida de forma que no limite não relativístico sejam obtidos os resultados previstos pelas leis de Newton. É bastante comum na literatura, como por exemplo em [25], o uso das chamadas "unidades geométricas", em que $G_{N}=c=1$. Neste sistema de unidades, usado deste ponto em diante, o fator $8 \pi G_{N} / c^{4}$ torna-se $8 \pi$. 


\section{Capítulo 3}

\section{Universos Brana}

Neste capítulo, discutiremos possíveis dimensões extras, exploraremos modelos de mundos brana, com destaque aos modelos Randall-Sundrum, e, na seção final, obteremos a projeção das equações de Einstein generalizadas na brana.

\subsection{Dimensões extras}

A utilização de dimensões extras para descrever fenômenos físicos foi feita pioneiramente por Kaluza e Klein nos trabalhos $[1,2]$ e, atualmente, é feita pela Teoria de Cordas. Busca-se, conseqüentemente, a influência destas dimensões extras no nosso mundo 4-dimensional e modificações em Teorias Físicas.

Segundo a lei da Gravitação Universal de Newton, o módulo da força entre duas partículas de massas $m_{1}$ e $m_{2}$, separadas pela distância $r$, é dado por $G_{N} m_{1} m_{2} / r^{2}$, onde $G_{N}$ é a constante gravitacional newtoniana. O fato da força depender somente da distância relativa entre os corpos pode ser creditado como uma conseqüência da isotropia do espaço, ou seja, as leis físicas são independentes da direção, são as mesmas em qualquer direção. Quando adota-se $h / 2 \pi=c=1$ (onde $h$ é a constante de Planck e $c$ é a velocidade da luz), $G_{N}=\left(10^{-33} \mathrm{~cm}\right)^{2}$ é inversamente proporcional a $M_{p}^{2}$, onde $M_{p}=$ $10^{19} \mathrm{Gev}$ é a massa de Planck. Dessa forma, temos uma grandeza $\left(G_{N}=10^{-66} \mathrm{~cm}^{2}\right)$ muito pequena, incapaz de ser observada atualmente.

Para o caso de objetos astronômicos, objetos macroscópicos, a lei da gravitação diminui com $1 / r^{2}$ e funciona muito bem. Quando são feitas medidas em pequenas distâncias, a influência de outras forças, como a força elétrica por exemplo, interfere na 
obtenção de resultados. Isso é devido ao fato de que a lei da gravitação é fraca a curtas distâncias. Os melhores dados obtidos por [6] mostram que abaixo de $0,1 \mathrm{~mm}$ não podemos discernir entre a força gravitacional e as demais forças. Então, a lei $1 / r^{2}$ funciona bem entre escalas astronômicas, distâncias da ordem do sistema solar, e 0,1 $\mathrm{mm}$. Caso dimensões extras existam, sua importância seria notada em medidas abaixo de 0,1 $\mathrm{mm}$. Além disso, com uma dimensão espacial extra, a gravitação seria notada em todas dimensões e diminuiria com $1 / r^{3}$. De uma forma geral, num mundo $n$-dimensional, a lei da gravitação diminuiria com $1 / r^{n-2}$ e seria inversamente proporcional à massa de Planck $n$-dimensional. Assim, a massa de Planck 4-dimensional seria relacionada com a $n$-dimensional por

$$
M_{p}^{2}=M^{n-2} V,
$$

onde $V$ é o volume das $n-4$ dimensões adicionais.

Em 1921, Kaluza [1] na sua tentativa de unificar o eletromagnetismo com a gravitação propôs um espaço-tempo com 4 dimensões espaciais, isto é, 3 dimensões infinitas e uma quarta compacta presa num círculo de tamanho $r_{c}$ na dimensão extra. Klein num trabalho em 1926 [2], determinou que o tamanho desse círculo seria da ordem da escala de Planck, $10^{-33} \mathrm{~cm}$, algo totalmente não observável. Dessa forma, a lei da gravitação é consistente com o que observamos, mesmo com a isotropia do espaço violada, ou seja, as demais dimensões são distinguidas da dimensão extra pois as linhas de força só podem se propagar até uma distância $r_{c}$ na dimensão compacta. Sendo a massa de Planck inversamente proporcional à $G_{N}$, temos em largas distâncias, com $r>r_{c}$, a adequação da lei da gravitação a um espaço-tempo 4-dimensional (3 dimensões espaciais e 1 dimensão temporal) e sua diminuição com $1 /\left(M_{p} r\right)^{2}$. Em curtas distâncias, $r<r_{c}$, a lei da força diminui com $1 /(M r)^{n-2}$.

Até o momento, nos referimos a dimensões extras de tamanho muito reduzido, da ordem da escala de Planck. Para Teoria de Cordas, as 6 dimensões extras teriam esta ordem de grandeza. Entretanto, é possível pensar numa dimensão não tão reduzida e até infinita, como no modelo Randall-Sundrum. Restrições experimentais (veja [7]) indicam que tais dimensões extras podem ser menores que $10^{3} \mathrm{GeV}$, isto é, 16 ordens de grandeza maior do que a escala de Planck, $10^{19} \mathrm{GeV}$, e, além disso, através do trabalho de Arkani-Hamed, Dimopoulos e Dvali [8], que deu origem ao modelo ADD, a previsão para o raio da dimensão compacta no caso de 2 dimensões extras pode ser $1 \mathrm{~mm}$.

O modelo ADD utiliza um conceito proveniente da Teoria de Cordas que será modificado e utilizado nos modelos Randall-Sundrum, o conceito de brana. O termo brana 
deriva-se de membrana e significa uma superfície imersa num espaço com maior número de dimensões. Para Teoria de Cordas, existem subvariedades $p+1$-dimensionais imersas no bulk 10-dimensional chamadas $p$-branas. Entre as $p$-branas existem as $D$-branas, que são extremidades de cordas abertas, 1-brana. Assim, no modelo ADD temos um espaço tempo $4+d$ dimensional, com as $d$ dimensões extras compactas, contornado por branas onde todos os campos, exceto o gravitacional, estão confinados nas subvariedades. A motivação do modelo ADD foi resolver o problema da hierarquia, que é a diferença entre a escala de massa eletrofaca, $M_{f} \sim 10^{3} \mathrm{GeV}$, e a escala de massa de Planck 4-dimensional, $M_{p} \sim 10^{19} \mathrm{GeV}$.

Entretanto, a dimensão extra pode ser maior do que alguns milímetros, pode ser infinita como veremos no modelo Randall-Sundrum 2 (RS-II).

\subsection{Modelos Randall-Sundrum}

Existem dois modelos Randall-Sundrum, ambos utilizam a idéia de um bulk de 5 dimensões com todos campos, exceto o gravitacional, confinados na brana. O modelo RS-I consiste de duas branas 4-dimensionais imersas no bulk com o tamanho da dimensão extra finito. O modelo RS-II consiste de uma brana 4-dimensional imersa no bulk com o tamanho da dimensão extra podendo ser até infinito. Os modelos Randall-Sundrum foram desenvolvidos com o intuito de resolver o problema da hierarquia.

Como veremos, devido ao fato de transportar energia, a gravidade será mais forte perto da brana e diminuirá exponencialmente quando nos afastarmos dela na dimensão extra. Dessa forma, a métrica na brana terá um fator exponencial que dependerá dessa nova dimensão e, assim, não será fatorável. Esse novo fator na métrica atenua a gravidade e corrige o problema da hierarquia.

\section{Randall-Sundrum I}

De acordo com [9], o modelo RS-I adota duas 3-branas imersas num bulk 5-dimensional anti-de Sitter, cuja coordenada extra, $y$, é compacta com raio $r_{c}$. Além disso, o bulk possui simetria $S^{1} / \mathbb{Z}_{2}$. Uma das branas pode ser identificada como nosso universo visível e a outra como um universo escondido. Ambas possuem posições fixas no bulk e podem ser interpretadas como condições de contorno neste espaço 5-dimensional. Assim, pode- 
mos restringir o intervalo de interesse da coordenada $y$ colocando uma brana em $y=0$, a brana visível, e a outra em $y=\pi r_{c}$, a brana escondida. Na figura 3.1, é representada uma imagem deste modelo com uma dimensão espacial suprimida.

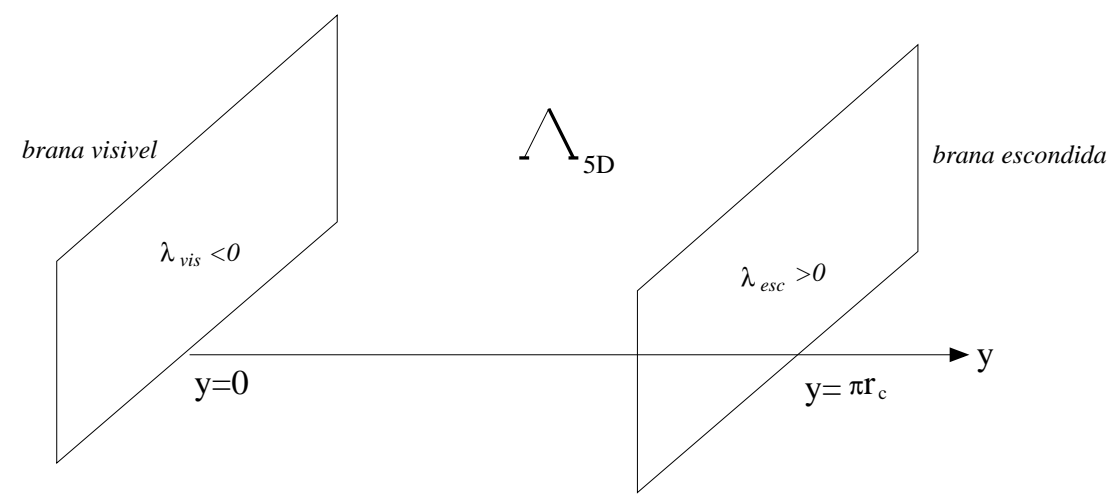

Figura 3.1: Representação do modelo RS-I onde uma dimensão espacial foi suprimida. As duas branas, visível e escondida, possuem tensão negativa e positiva, respectivamente. Ambas estão imersas num bulk anti-de Sitter com constante cosmológica $\Lambda_{5 D}$.

Então, podemos escrever as métricas das duas 3-branas como

$$
\begin{gathered}
g_{v i s}^{a b}=G^{\mu \nu}(y=0) \delta_{\mu}^{a} \delta_{\nu}^{b}, \\
g_{\text {esc }}^{a b}=G^{\mu \nu}\left(y=\pi r_{c}\right) \delta_{\mu}^{a} \delta_{\nu}^{b},
\end{gathered}
$$

onde $G^{\mu \nu}$ é a métrica no bulk, os índices gregos vão de 0 a 4 e os índices latinos vão de 0 a 3.

A ação clássica que descreve este modelo é dada por

$$
S=S_{5 D}+S_{v i s}+S_{e s c}
$$

sendo $S_{5 D}, S_{v i s}, S_{e s c}$ as ações no bulk, brana visível e brana escondida, respectivamente. Podemos escrevê-las da seguinte forma

$$
\begin{aligned}
& S_{5 D}=\int \sqrt{|G|}\left[-\Lambda_{5 D}+2 M_{5 D}^{3} R\right] d^{4} x d y \\
& \left.S_{v i s}=\int \sqrt{\left|g_{v i s}\right|} \mid \mathcal{L}_{v i s}-2 \lambda_{v i s}\right] \delta(y) d^{4} x d y
\end{aligned}
$$

e

$$
S_{e s c}=\int \sqrt{\left|g_{e s c}\right|}\left[\mathcal{L}_{e s c}-2 \lambda_{e s c}\right] \delta\left(y-\pi r_{c}\right) d^{4} x d y
$$


onde $G, g_{v i s}$ e $g_{e s c}$ são os determinantes das métricas do bulk, brana visível e brana escondida, respectivamente. $\Lambda_{5 D}$ e $M_{5 D}$ são a constante cosmológica e a massa de Planck em 5 dimensões, respectivamente. $R$ é o escalar de Ricci, $\lambda_{v i s}$ é a tensão na brana visível e $\lambda_{e s c}$ é a tensão na brana escondida, ambas atenuam o efeito da constante cosmológica do bulk na brana. As lagrangeanas $\mathcal{L}_{\text {vis }}$ e $\mathcal{L}_{\text {esc }}$ das 3-branas não precisam ser conhecidas em detalhes para se determinar o métrica do bulk. As integrais acima são em relação a $x^{a}$, coordenada na brana, e $y$, coordenada da dimensão extra.

Podemos obter as equações de Einstein em 5 dimensões extremizando a ação (3.4). Dessa forma, as equações de campo são

$$
\begin{gathered}
\sqrt{|G|}\left(R^{\mu \nu}-\frac{1}{2} G^{\mu \nu} R\right)=-\frac{1}{4 M_{5 D}^{3}}\left[2 \sqrt{\left|g_{v i s}\right|} g_{v i s}^{a b} \delta_{a}^{\mu} \delta_{b}^{\nu} \lambda_{v i s} \delta(y)\right. \\
\left.+\sqrt{|G|} G^{\mu \nu} \Lambda_{5 D}+2 \sqrt{\left|g_{e s c}\right|} g_{e s c}^{a b} \delta_{a}^{\mu} \delta_{b}^{\nu} \lambda_{e s c} \delta\left(y-\pi r_{c}\right)\right] .
\end{gathered}
$$

Assumindo que existe uma solução quadridimensional que obedece a invariância de Poincaré nas direções $x^{a}$, quando a geometria é plana (Minkowski), pode-se usar a métrica 5-dimensional

$$
d s^{2}=e^{2 \sigma(y)} \eta_{a b} d x^{a} d x^{b}+d y^{2}
$$

como Ansatz em (3.8). Assim, as equações de Einstein reduzem-se a

$$
6 \sigma^{\prime 2}=-\frac{\Lambda_{5 D}}{4 M_{5 D}^{3}}
$$

$\mathrm{e}$

$$
3 \sigma^{\prime \prime}=\frac{\lambda_{v i s} \delta(y)}{2 M_{5 D}^{3}}+\frac{\lambda_{e s c} \delta\left(y-\pi r_{c}\right)}{2 M_{5 D}^{3}} .
$$

Os símbolos linha "'” indicam derivação com respeito a $y$. A solução para (3.10) que respeita a simetria $\mathbb{Z}_{2}, y \rightarrow-y$, é

$$
\sigma= \pm|y| \sqrt{-\frac{\Lambda_{5 D}}{24 M_{5 D}^{3}}} .
$$

Vemos em (3.12) que a solução aceitável é aquela com $\Lambda_{5 D}$ negativa. Dessa forma, o espaço-tempo entre as duas branas é do tipo $A d S_{5}$.

Como a métrica (3.9) é uma função periódica em $y$, se derivarmos (3.12), válida no 
intervalo $-\pi r_{c} \leq y \leq \pi r_{c}$, obtemos

$$
\sigma^{\prime \prime}= \pm 2 r_{c} \sqrt{-\frac{\Lambda_{5 D}}{24 M_{5 D}^{3}}}\left\{\delta(y)-\delta\left(y-\pi r_{c}\right)\right\}
$$

Então, obtemos (3.11) a partir de (3.13) se $\lambda_{v i s}, \lambda_{\text {esc }}$ e $\Lambda_{5 D}$ estiverem relacionadas com um escalar $k$ da seguinte maneira

$$
\lambda_{v i s}=-\lambda_{e s c}=\mp 12 M_{5 D}^{3} k \quad \text { e } \quad \Lambda_{5 D}=-24 M^{3} k^{2} .
$$

As relações acima entre os termos cosmológicos do bulk e da borda são fundamentais para se obter uma solução que respeite a invariância de Poincaré 4-dimensional na brana. Com isso, a métrica do bulk é

$$
d s^{2}=e^{ \pm 2 k|y|} \eta_{a b} d x^{a} d x^{b}+d y^{2}
$$

Para ambos os modelos, RS-I e RS-II, a métrica do bulk é a mesma com a diferença de que para o modelo número um o sinal da exponencial é positivo e para o modelo número dois o sinal é negativo. Para o RS-I, o crescimento da exponencial na direção $y$ é barrado pela brana escondida.

A constante de Newton para a brana visível é dada por

$$
G_{4 D}=\frac{\lambda_{v i s}}{6 M_{5 D}^{6}}
$$

onde a tensão negativa na brana visível induz uma gravidade repulsiva na mesma. Na brana escondida a tensão é positiva.

\section{Randall-Sundrum II}

No modelo Randall-Sundrum II, de acordo com [10], apenas uma 3-brana é utilizada e, como no RS-I, o bulk tem uma geometria anti-de Sitter, $A d S_{5}$, com simetria $\mathbb{Z}_{2}$. A coordenada $y$ pode tender ao infinito, ou seja, ao contrário do modelo anterior, o RS-II não utiliza uma dimensão extra compacta e, dessa forma, $r_{c} \rightarrow \infty$.

A métrica do bulk no RS-II é a mesma do RS-I com sinal negativo na exponencial. A maneira que se obteve tal resultado é semelhante ao RS-I e, conseqüentemente, 
omitiremos os cálculos. Assim, a métrica no bulk para esse modelo é

$$
d s^{2}=e^{-2 k|y|} \eta_{a b} d x^{a} d x^{b}+d y^{2} .
$$

A vantagem do RS-II reside no fato de que a única brana, a brana visível, tem tensão positiva, ou seja, possui uma gravidade atrativa.

Fazendo $k=1 / l$ e introduzindo a coordenada $z=l e^{y / l} \mathrm{em}(3.17)$, obtemos

$$
d s^{2}=\frac{l^{2}}{z^{2}}\left(d z^{2}+\eta_{a b} d x^{a} d x^{b}\right),
$$

onde $l$ é o raio AdS. A métrica de Minkowski em (3.18) pode ser substituída por qualquer métrica Ricci plana e será também solução de (3.8) como pode ser visto em [26].

\subsection{Projeção das equações de Einstein na 3-brana}

A partir de agora, vamos obter as equações de Einstein na 3-brana (imersa num bulk 5-dimensional com simetria $\mathbb{Z}_{2}$ ) com auxílio das equações de Gauss e Codacci, de acordo com [16]. Vamos, então, relacionar o tensor de Einstein na brana com elementos no bulk. Novamente, todos os campos, exceto o gravitacional, estão confinados na brana. As equações na 3-brana se reduzem às equações de Einstein convencionais no limite de baixas energias. Os índices gregos vão de 0 a 3 para tensores em 4 dimensões e de $0 \mathrm{a}$ 4 para tensores em 5 dimensões (a dimensionalidade será indicada pelos números entre parênteses inseridos no lado superior esquerdo do tensor). Esta seção foi diretamente influenciada pelo capítulo 6 de [27].

Nosso universo, uma brana 4-dimensional, será indicada por $\mathcal{M}$ e terá uma métrica dada por

$$
q_{\mu \nu}=g_{\mu \nu}-n_{\mu} n_{\nu} \quad \text { e } \quad q^{\mu \nu}=g^{\mu \nu}-n^{\mu} n^{\nu},
$$

onde $g_{\mu \nu}$ é a métrica no bulk e $n^{\alpha}$ é um vetor unitário normal à brana. Podemos escrever algumas propriedades que nos ajudarão na obtenção das equações desejadas

$$
q_{\mu}^{\nu}=g_{\mu}^{\nu}-n_{\mu} n^{\nu}, \quad n_{\mu} n^{\mu}=1 \quad \text { e } \quad q_{\mu \nu} n^{\nu}=0
$$

sendo $q_{\mu}^{\nu}$ um operador de projeção, isto é, dado um campo qualquer, $X^{\mu}$, no bulk, $q_{\mu}^{\nu}$ calcula sua projeção na brana. 
As equações de Gauss e Codacci podem ser escritas como

$$
{ }^{(4)} R_{\beta \gamma \delta}^{\alpha}{ }^{(5)} R^{\mu}{ }_{\nu \rho \sigma} q_{\mu}^{\alpha} q_{\beta}{ }^{\nu} q_{\gamma}{ }^{\rho} q_{\delta}{ }^{\sigma}+K_{\gamma}{ }^{\alpha} K_{\beta \delta}-K_{\delta}{ }^{\alpha} K_{\beta \gamma}
$$

e

$$
D_{\nu} K_{\mu}{ }^{\nu}-D_{\mu} K={ }^{(5)} R_{\rho \sigma} \eta^{\sigma} q_{\mu}{ }^{\rho},
$$

respectivamente, onde $K_{\mu \nu}=q_{\mu}{ }^{\alpha} q_{\nu}{ }^{\beta} \nabla_{\alpha} n_{\beta}$ é a curvatura extrínseca de $\mathcal{M}, K_{\mu}{ }^{\mu}=K$ é o traço de $K_{\mu \nu}$ e $D_{\mu}$ é o operador derivada covariante em relação à métrica $q_{\mu \nu}$. Contraindo (3.21) com relação aos índices $\alpha$ e $\gamma$, obtemos

$$
{ }^{(4)} R_{\beta \delta}={ }^{(5)} R^{\mu}{ }_{\nu \sigma \sigma} q_{\mu}{ }^{\alpha} q_{\beta}{ }^{\nu} q_{\alpha}{ }^{\rho} q_{\delta}{ }^{\sigma}+K K_{\beta \delta}-K_{\delta}{ }^{\alpha} K_{\beta \alpha},
$$

$\operatorname{como} q_{\mu}^{\alpha}=g_{\mu}^{\alpha}-n_{\mu} n^{\alpha}$ e $q_{\alpha}^{\rho}=g_{\alpha}^{\rho}-n_{\alpha} n^{\rho}$, (3.23) fica

$$
\begin{aligned}
{ }^{(4)} R_{\beta \delta} & ={ }^{(5)} R^{\mu}{ }_{\nu \rho \sigma}\left(g_{\mu}^{\alpha}-n_{\mu} n^{\alpha}\right)\left(g_{\alpha}{ }^{\rho}-n_{\alpha} n^{\rho}\right) q_{\beta}{ }^{\nu} q_{\delta}{ }^{\sigma}+K K_{\beta \delta}-K_{\delta}{ }^{\alpha} K_{\beta \alpha} \\
& ={ }^{(5)} R^{\mu}{ }_{\nu \rho \sigma}\left(g_{\mu}{ }^{\alpha} g_{\alpha}{ }^{\rho}-g_{\mu}{ }^{\alpha} n_{\alpha} n^{\rho}-g_{\alpha}{ }^{\rho} n_{\mu} n^{\alpha}+n_{\mu} n^{\alpha} n_{\alpha} n^{\rho}\right) q_{\beta}{ }^{\nu} q_{\delta}{ }^{\sigma}+ \\
& +K K_{\beta \delta}-K_{\delta}{ }^{\alpha} K_{\beta \alpha} .
\end{aligned}
$$

Usando as propriedades (3.20), (3.24) pode ser escrita como

$$
\begin{aligned}
{ }^{(4)} R_{\beta \delta} & ={ }^{(5)} R^{\mu}{ }_{\nu \rho \sigma}\left(g_{\mu}^{\alpha} g_{\alpha}{ }^{\rho}-n_{\mu} n^{\rho}\right) q_{\beta}{ }^{\nu} q_{\delta}{ }^{\sigma}+K K_{\beta \delta}-K_{\delta}{ }^{\alpha} K_{\beta \alpha}, \\
& ={ }^{(5)} R_{\nu \sigma} q_{\beta}{ }^{\nu} q_{\delta}{ }^{\sigma}-{ }^{(5)} R^{\mu}{ }_{\nu \rho \sigma} n_{\mu} n^{\rho} q_{\beta}^{\nu} q_{\delta}{ }^{\sigma}+K K_{\beta \delta}-K_{\delta}{ }^{\alpha} K_{\beta \alpha} .
\end{aligned}
$$

Fazendo mudanças de índices, obtemos

$$
{ }^{(4)} R_{\mu \nu}={ }^{(5)} R_{\delta \sigma} q_{\mu}{ }^{\delta} q_{\nu}{ }^{\sigma}-{ }^{(5)} R_{\delta \rho \sigma}^{\beta} n_{\beta} n^{\rho} q_{\mu}{ }^{\delta} q_{\nu}{ }^{\sigma}+K K_{\mu \nu}-K_{\nu}{ }^{\alpha} K_{\alpha \mu}
$$

Agora que temos o tensor de Ricci em 4 dimensões, podemos obter o escalar de Ricci contraindo (3.25) com a métrica $q^{\mu \nu}$

$$
\begin{gathered}
{ }^{(4)} R=q^{\mu \nu(4)} R_{\mu \nu} \\
{ }^{(4)} R={ }^{(5)} R_{\delta \sigma} q^{\delta \sigma}-q^{\mu \nu{ }^{(5)}} R_{\delta \rho \sigma}^{\beta} n_{\beta} n^{\rho} q_{\mu}^{\delta} q_{\nu}{ }^{\sigma}+K^{2}-K^{\mu \alpha} K_{\mu \alpha} .
\end{gathered}
$$

Dessa forma, com auxílio de (3.25) e (3.26), as equações de Einstein ficam 


$$
\begin{aligned}
{ }^{(4)} G_{\mu \nu}= & { }^{(4)} R_{\mu \nu}-\frac{1}{2} q_{\mu \nu}{ }^{(4)} R, \\
= & { }^{(5)} R_{\delta \sigma} q_{\mu}{ }^{\delta} q_{\nu}{ }^{\sigma}-{ }^{(5)} R_{\delta \rho \sigma}^{\beta} n_{\beta} n^{\rho} q_{\mu}{ }^{\delta} q_{\nu}{ }^{\sigma}+K K_{\mu \nu}-K_{\nu}{ }^{\alpha} K_{\alpha \mu}- \\
& \left.-\frac{1}{2} q_{\mu \nu}{ }^{(5)} R_{\delta \sigma} q^{\delta \sigma}-q^{\mu \nu}{ }^{(5)} R_{\delta \rho \sigma}^{\beta} n_{\beta} n^{\rho} q_{\mu}{ }^{\delta} q_{\nu}{ }^{\sigma}+K^{2}-K^{\mu \alpha} K_{\mu \alpha}\right],
\end{aligned}
$$

após alguma manipulação algébrica, temos

$$
\begin{aligned}
{ }^{(4)} G_{\mu \nu} & ={ }^{(5)} R_{\delta \sigma} q_{\mu}{ }^{\delta} q_{\nu}{ }^{\sigma}-{ }^{(5)} R_{\delta \sigma} q^{\delta \sigma} \frac{1}{2} q_{\mu \nu}-\tilde{E}_{\mu \nu}+K K_{\mu \nu}-K_{\nu}{ }^{\alpha} K_{\mu \alpha}- \\
& -\frac{1}{2} q_{\mu \nu}\left[K^{2}-K^{\mu \alpha} K_{\mu \alpha}-q^{\mu \nu}{ }^{(5)} R_{\delta \rho \sigma}^{\beta} n_{\beta} n^{\rho} q_{\mu}{ }^{\delta} q_{\nu}{ }^{\sigma}\right]
\end{aligned}
$$

onde

$$
\tilde{E}_{\mu \nu}={ }^{(5)} R_{\delta \rho \sigma}^{\beta} n_{\beta} n^{\rho} q_{\mu}^{\delta} q_{\nu}{ }^{\sigma} .
$$

Como $q_{\mu \nu}=q_{\delta \sigma} q_{\mu}{ }^{\delta} q_{\nu}{ }^{\sigma}$, o segundo termo do lado direito de (3.27) pode ser escrito da seguinte forma

$$
\begin{aligned}
-\frac{1}{2}^{(5)} R_{\delta \sigma} q^{\delta \sigma} q_{\mu \nu} & =-\left(\frac{1}{2} q_{\delta \sigma}{ }^{(5)} R_{\delta \sigma} q^{\delta \sigma}\right) q_{\mu}^{\delta} q_{\nu}{ }^{\sigma} \\
& =\left[-\frac{1}{2}\left(g_{\delta \sigma}-n_{\delta} n^{\sigma}\right){ }^{(5)} R_{\delta \sigma}\left(g^{\delta \sigma}-n^{\delta} n^{\sigma}\right)\right] q_{\mu}^{\delta} q_{\nu}^{\sigma} \\
& =\left[-\frac{1}{2}\left(g_{\delta \sigma}-n_{\delta} n^{\sigma}\right)\left({ }^{(5)} R-{ }^{(5)} R_{\delta \sigma} n^{\delta} n^{\sigma}\right)\right] q_{\mu}^{\delta} q_{\nu}^{\sigma}
\end{aligned}
$$

então,

$$
\begin{aligned}
-\frac{1^{(5)}}{2} R_{\delta \sigma} q^{\delta \sigma} q_{\mu \nu} & =-\frac{1}{2} g_{\delta \sigma}{ }^{(5)} R q_{\mu}{ }^{\delta} q_{\nu}{ }^{\sigma}+\frac{1}{2} R_{\delta \sigma} g_{\delta \sigma} n^{\delta} n^{\sigma} q_{\mu}{ }^{\delta} q_{\nu}{ }^{\sigma}+ \\
& +\frac{1}{2} n_{\delta} n_{\sigma}{ }^{(5)} R q_{\mu}{ }^{\delta} q_{\nu}{ }^{\sigma}-\frac{1}{2} n_{\delta} n_{\sigma} n^{\delta} n^{\sigma}{ }^{(5)} R_{\delta \sigma} q_{\mu}{ }^{\delta} q_{\nu}{ }^{\sigma}
\end{aligned}
$$

Usando (3.20) e o fato de que ${ }^{(5)} R=g^{\delta \sigma(5)} R_{\delta \sigma}$, (3.29) fica

$$
-\frac{1}{2}^{(5)} R_{\delta \sigma} q^{\delta \sigma} q_{\mu \nu}=-\frac{1}{2} g_{\delta \sigma}{ }^{(5)} R q_{\mu}^{\delta} q_{\nu}{ }^{\sigma}+\frac{1}{2} R_{\delta \sigma} n^{\delta} n^{\sigma} q_{\mu \nu}
$$

Assim, substituindo (3.30) em (3.27), obtemos

$$
\begin{aligned}
{ }^{(4)} G_{\mu \nu} & =\left({ }^{(5)} R_{\delta \sigma}-\frac{1}{2} g_{\delta \sigma}{ }^{(5)} R\right) q_{\mu}{ }^{\delta} q_{\nu}{ }^{\sigma}+\frac{1}{2} q_{\mu \nu}{ }^{(5)} R_{\delta \sigma} n^{\delta} n^{\sigma}-\tilde{E}_{\mu \nu}+ \\
& +K K_{\mu \nu}-K_{\nu}{ }^{\alpha} K_{\mu \alpha}-\frac{1}{2} q_{\mu \nu}\left[K^{2}-K^{\mu \alpha} K_{\mu \alpha}-q^{\mu \nu}{ }^{(5)} R_{\delta \rho \sigma}^{\beta} n_{\beta} n^{\rho} q_{\mu}{ }^{\delta} q_{\nu}{ }^{\sigma}\right]
\end{aligned}
$$


sabendo que ${ }^{(5)} G_{\delta \sigma}={ }^{(5)} R_{\delta \sigma}-\frac{1}{2} g_{\delta \sigma}{ }^{(5)} R$, então

$$
\begin{aligned}
{ }^{(4)} G_{\mu \nu} & ={ }^{{ }^{(5)}} G_{\delta \sigma} q_{\mu}{ }^{\delta} q_{\nu}{ }^{\sigma}-\tilde{E}_{\mu \nu}+K K_{\mu \nu}-K_{\nu}{ }^{\alpha} K_{\mu \alpha}+ \\
& +\frac{1}{2} q_{\mu \nu}\left[R_{\delta \sigma} n^{\delta} n^{\sigma}-K^{2}+K^{\mu \alpha} K_{\mu \alpha}+R_{\delta \rho \sigma}^{\beta} n_{\beta} n^{\rho} q^{\delta \sigma}\right] .
\end{aligned}
$$

Novamente, com auxílio de (3.19) podemos escrever (3.31) da seguinte forma

$$
\begin{aligned}
{ }^{(4)} G_{\mu \nu} & ={ }^{(5)} G_{\delta \sigma} q_{\mu}{ }^{\delta} q_{\nu}{ }^{\sigma}-\tilde{E}_{\mu \nu}+K K_{\mu \nu}-K_{\nu}{ }^{\alpha} K_{\mu \alpha}+ \\
& +\frac{1}{2} q_{\mu \nu}\left[K^{\mu \alpha} K_{\mu \alpha}-K^{2}+R_{\delta \sigma} n^{\delta} n^{\sigma}+{ }^{(5)} R_{\delta \rho \sigma}^{\beta} n_{\beta} n^{\rho}\left(g^{\delta \sigma}-n^{\delta} n^{\sigma}\right)\right]
\end{aligned}
$$

ou seja,

$$
\begin{gathered}
{ }^{(4)} G_{\mu \nu}={ }^{(5)} G_{\delta \sigma} q_{\mu}{ }^{\delta} q_{\nu}{ }^{\sigma}-\tilde{E}_{\mu \nu}+K K_{\mu \nu}-K_{\nu}{ }^{\alpha} K_{\mu \alpha}+ \\
\left.+\frac{1}{2} q_{\mu \nu}\left[K^{\mu \alpha} K_{\mu \alpha}-K^{2}+R_{\delta \sigma} n^{\delta} n^{\sigma}+{ }^{(5)} R^{\beta}{ }_{\rho} n_{\beta} n^{\rho}-{ }^{(5)} R_{\delta \rho \sigma}^{\beta} n_{\beta} n^{\rho} n^{\delta} n^{\sigma}\right)\right]
\end{gathered}
$$

Fazendo

$$
{ }^{(5)} R_{\rho}^{\beta} n_{\beta} n^{\rho}=g^{\beta \delta(5)} R_{\delta \rho} n_{\beta} n^{\rho}={ }^{(5)} R_{\delta \rho} n^{\delta} n^{\rho}={ }^{(5)} R_{\delta \sigma} n^{\delta} n^{\sigma}
$$

e

$$
{ }^{(5)} R_{\delta \rho \sigma}^{\beta} n_{\beta} n^{\rho} n^{\delta} n^{\sigma}=g^{\beta \beta(5)} R_{\beta \delta \rho \sigma} n_{\beta} n^{\rho} n^{\delta} n^{\sigma}={ }^{(5)} R_{\beta \delta \rho \sigma} n^{\beta} n^{\delta} n^{\rho} n^{\sigma}=0,
$$

pois ${ }^{(5)} R_{\beta \delta \rho \sigma}$ é um tensor anti-simétrico e $n^{\beta} n^{\delta} n^{\rho} n^{\sigma}$ é um tensor simétrico, o tensor 4-dimensional em (3.32) fica

$$
\begin{aligned}
{ }^{(4)} G_{\mu \nu}= & { }^{(5)} G_{\delta \sigma} q_{\mu}{ }^{\delta} q_{\nu}{ }^{\sigma}-\tilde{E}_{\mu \nu}+K K_{\mu \nu}-K_{\nu}{ }^{\alpha} K_{\mu \alpha}+ \\
& +q_{\mu \nu}{ }^{(5)} R_{\delta \sigma} n^{\delta} n^{\sigma}-\frac{1}{2} q_{\mu \nu}\left(K^{2}-K^{\mu \alpha} K_{\mu \alpha}\right) .
\end{aligned}
$$

Vamos, a partir de agora, relacionar ${ }^{(4)} G_{\mu \nu}$ com o tensor energia-momento na brana. Para isso, vamos usar as equações de Einstein em 5 dimensões

$$
{ }^{(5)} G_{\delta \sigma}={ }^{(5)} R_{\delta \sigma}-\frac{1}{2} g_{\delta \sigma}{ }^{(5)} R=\kappa_{5}^{2}{ }^{(5)} T_{\delta \sigma},
$$

onde $\kappa_{5}^{2}$ é a constante gravitacional em 5 dimensões e ${ }^{(5)} T_{\delta \sigma}$ é o tensor energia-momento 
do bulk. Usaremos também o tensor de Weyl em 5 dimensões

$$
\begin{aligned}
{ }^{(5)} C_{\alpha \beta \gamma \delta} & ={ }^{(5)} R_{\alpha \beta \gamma \delta}-\frac{1}{3}\left(g_{\alpha \gamma}{ }^{(5)} R_{\delta \beta}-g_{\alpha \delta}{ }^{(5)} R_{\gamma \beta}-g_{\beta \gamma}{ }^{(5)} R_{\delta \alpha}+g_{\beta \delta}{ }^{(5)} R_{\gamma \alpha}\right)+ \\
& +\frac{1}{12}\left(g_{\alpha \gamma} q_{\delta \beta}-g_{\alpha \delta} g_{\gamma \beta}\right){ }^{(5)} R .
\end{aligned}
$$

A partir de (3.36), obtemos, com auxílio de $g^{\delta \sigma}$,

$$
{ }^{(5)} R=-\frac{2}{3} \kappa_{5}^{2}{ }^{(5)} T \quad \text { e } \quad{ }^{(5)} R_{\delta \sigma}=\frac{\kappa_{5}^{2}}{3}\left({ }^{(5)} T_{\delta \sigma}-{ }^{(5)} T g_{\delta \sigma}\right)
$$

ou seja, o escalar e o tensor de Ricci em 5 dimensões. Substituindo-os na equação (3.35), obtemos

$$
\begin{aligned}
{ }^{(4)} G_{\mu \nu} & =\kappa_{5}^{2}{ }^{(5)} T_{\delta \sigma} q_{\mu}{ }^{\delta} q_{\nu}{ }^{\sigma}-\tilde{E}_{\mu \nu}+K K_{\mu \nu}-K_{\nu}{ }^{\alpha} K_{\mu \alpha}+ \\
& +q_{\mu \nu} \frac{\kappa_{5}^{2}}{3}\left({ }^{(5)} T_{\delta \sigma}-{ }^{(5)} T g_{\delta \sigma}\right) n^{\delta} n^{\sigma}-\frac{1}{2} q_{\mu \nu}\left(K^{2}-K^{\mu \alpha} K_{\mu \alpha}\right) .
\end{aligned}
$$

Podemos relacionar $\tilde{E}_{\mu \nu}$ com ${ }^{(5)} C_{\alpha \beta \gamma \delta}$ usando (3.37). Sabemos que

$$
\begin{aligned}
\tilde{E}_{\mu \nu} & ={ }^{(5)} R_{\delta \rho \sigma}^{\beta} n_{\beta} n^{\rho} q_{\mu}{ }^{\delta} q_{\nu}{ }^{\sigma}, \\
& =g^{\beta \beta}{ }^{(5)} R_{\beta \delta \rho \sigma} n_{\beta} n^{\rho} q_{\mu}^{\delta} q_{\nu}{ }^{\sigma}, \\
& ={ }^{(5)} R_{\beta \delta \rho \sigma} n^{\beta} n^{\rho} q_{\mu}{ }^{\delta} q_{\nu}{ }^{\sigma},
\end{aligned}
$$

depois de trocas de índices temos

$$
\tilde{E}_{\mu \nu}={ }^{(5)} R_{\alpha \beta \gamma \delta} n^{\alpha} n^{\gamma} q_{\mu}^{\beta} q_{\nu}^{\delta} .
$$

Multiplicando (3.37) por $n^{\alpha} n^{\delta} q_{\mu}{ }^{\beta} q_{\nu}^{\delta}$, e com o auxílio de (3.40) e (3.20), teremos

$$
\begin{aligned}
\tilde{E}_{\mu \nu} & =\frac{1}{3}\left({ }^{(5)} R_{\delta \beta} q_{\mu}{ }^{\beta} q_{\nu}{ }^{\delta}+{ }^{(5)} R_{\gamma \alpha} n^{\gamma} n^{\alpha} g_{\beta \delta} q_{\mu}{ }^{\beta} q_{\nu}{ }^{\delta}\right)-\frac{1}{12}\left({ }^{(5)} R g_{\delta \beta} q_{\mu}{ }^{\beta} q_{\nu}{ }^{\delta}\right)+ \\
& +{ }^{(5)} C_{\alpha \beta \gamma \delta} n^{\alpha} n^{\gamma} q_{\mu}{ }^{\beta} q_{\nu}{ }^{\delta},
\end{aligned}
$$

e com ajuda de (3.38), $\tilde{E}_{\mu \nu}$ fica

$$
\begin{aligned}
\tilde{E}_{\mu \nu} & =\frac{1}{3}\left[\frac{\kappa_{5}^{2}}{3}\left(3^{(5)} T_{\delta \beta}-{ }^{(5)} T g_{\delta \beta}\right) q_{\mu}{ }^{\beta} q_{\nu}{ }^{\delta}+\frac{\kappa_{5}^{2}}{3}\left({ }^{(5)} T_{\gamma \alpha}-{ }^{(5)} T_{\gamma \alpha}\right) n^{\alpha} n^{\gamma} g_{\beta \delta} q_{\mu}{ }^{\beta} q_{\nu}{ }^{\delta}\right]- \\
& -\frac{1}{12}\left(-\frac{2}{3} \kappa_{5}^{2}{ }^{(5)} T g_{\delta \beta} q_{\mu}{ }^{\beta} q_{\nu}{ }^{\delta}\right)+E_{\mu \nu},
\end{aligned}
$$




$$
\tilde{E}_{\mu \nu}=\frac{\kappa_{5}^{2}}{3}{ }^{(5)} T_{\delta \beta} q_{\mu}{ }^{\beta} q_{\nu}{ }^{\delta}-\frac{1}{6} \kappa_{5}^{2}{ }^{(5)} T g_{\delta \beta} q_{\mu}^{\beta} q_{\nu}{ }^{\delta}+\frac{1}{3} \kappa_{5}^{2}{ }^{(5)} T_{\gamma \alpha} n^{\alpha} n^{\gamma} q_{\mu \nu}+E_{\mu \nu},
$$

onde

$$
E_{\mu \nu}=C_{\alpha \beta \gamma \delta} n^{\alpha} n^{\gamma} q_{\mu}^{\beta} q_{\nu}^{\delta} .
$$

Uma propriedade de $E_{\mu \nu}$ é o fato de que seu traço é nulo, isto é, $E^{\mu}{ }_{\mu}=0$. Substituindo (3.41) em (3.39), obtemos as equações de Einstein 4-dimensionais em função do tensor energia-momento 5-dimensional

$$
\begin{aligned}
{ }^{(4)} G_{\mu \nu} & =-\kappa_{5}^{2}{ }^{(5)} T_{\delta \sigma} q_{\mu}{ }^{\delta} q_{\nu}{ }^{\sigma}+K K_{\mu \nu}-K_{\nu}{ }^{\alpha} K_{\mu \alpha}+\left(\frac{\kappa_{5}^{2}}{3}{ }^{(5)} T_{\delta \beta} q_{\mu}{ }^{\beta} q_{\nu}{ }^{\delta}-\frac{\kappa_{5}^{2}}{6}{ }^{(5)} T q_{\mu \nu}+\right. \\
& \left.+\frac{\kappa_{5}^{2}}{3}{ }^{(5)} T_{\gamma \alpha} n^{\alpha} n^{\gamma} q_{\mu \nu}+E_{\mu \nu}\right)+q_{\mu \nu} \frac{\kappa_{5}^{2}}{3}\left({ }^{(5)} T_{\delta \sigma}-{ }^{(5)} T g_{\delta \sigma}\right) n^{\delta} n^{\sigma}- \\
& -\frac{1}{2} q_{\mu \nu}\left(K^{2}-K^{\mu \alpha} K_{\mu \alpha}\right)
\end{aligned}
$$

e, logo em seguida, fazendo uma troca apropriada de índices mudos, obtemos

$$
\begin{aligned}
{ }^{(4)} G_{\mu \nu} & =\frac{2 \kappa_{5}^{2}}{3}\left({ }^{(5)} T_{\delta \sigma} q_{\mu}{ }^{\delta} q_{\nu}{ }^{\sigma}+\left[{ }^{(5)} T_{\delta \sigma} n^{\delta} n^{\sigma}-\frac{{ }^{(5)} T}{4}\right] q_{\mu \nu}\right)-E_{\mu \nu}+ \\
& +K K_{\mu \nu}-K_{\nu}{ }^{\alpha} K_{\mu \alpha}-\frac{1}{2} q_{\mu \nu}\left(K^{2}-K^{\mu \alpha} K_{\mu \alpha}\right) .
\end{aligned}
$$

A expressão (3.44) nos revela que as equações de Einstein em 4 dimensões, nesse cenário, possuem termos que não dependem do tensor energia-momento. Dessa forma, tais termos fazem o papel de uma matéria geométrica que curva o espaço-tempo.

A partir da equação (3.22), com o auxílio de (3.38) e do fato de que $g_{\delta \sigma} n^{\delta}=0$, obtemos a relação

$$
\begin{gathered}
D_{\nu} K_{\mu}{ }^{\nu}-D_{\mu} K=\frac{\kappa_{5}^{2}}{3}\left({ }^{(5)} T_{\delta \sigma}-{ }^{(5)} T g_{\delta \sigma}\right) \eta^{\delta} q_{\mu}{ }^{\sigma} \\
D_{\nu} K_{\mu}{ }^{\nu}-D_{\mu} K=\kappa_{5}^{2}{ }^{(5)} T_{\delta \sigma} n^{\delta} q_{\mu}{ }^{\sigma} .
\end{gathered}
$$

Até o presente momento, não utilizamos qualquer tipo de simetria em relação ao tensor energia-momento. A partir de agora, pensaremos num cenário brana e lançaremos mão de algumas escolhas. Comecemos com a escolha de uma coordenada $y$, a coordenada da dimensão extra, e adotaremos uma única brana, que será localizada em $y=0$. Assim, $n_{\mu} d x^{\mu}=d y$ e a métrica do bulk será dada por

$$
d s^{2}=q_{\mu \nu} d x^{\mu} d x^{\nu}+d y^{2} .
$$


Assumiremos que o tensor energia-momento no bulk tem a forma

$$
T_{\mu \nu}=-\Lambda_{5 D} g_{\mu \nu}+S_{\mu \nu} \delta(y),
$$

onde

$$
S_{\mu \nu}=-\lambda q_{\mu \nu}+\tau_{\mu \nu},
$$

sendo $\Lambda_{5 D}$ a constante cosmológica em 5 dimensões, $\lambda$ e $\tau_{\mu \nu}$ a tensão e o tensor energiamomento, respectivamente, na brana e $\tau_{\mu \nu} n^{\nu}=0$.

O tensor energia-momento $T_{\mu \nu}$ de acordo com (3.47) tem um comportamento singular devido à brana, que divide o bulk em duas partes, quebrando a simetria de translação em $y$. Assim, existe uma região de transição entre o bulk e a brana e isso requer uma solução de continuidade que é dada pelas condições de junção de Israel [11]:

$$
\left[q_{\mu \nu}\right]=0
$$

e

$$
\left[K_{\mu \nu}\right]=-\kappa_{5}^{2}\left(S_{\mu \nu}-\frac{1}{3} q_{\mu \nu} S\right),
$$

onde o operador $\left[X_{\mu \nu}\right]$ corresponde a

$$
\left[X_{\mu \nu}\right]=\lim _{y \rightarrow+0} X-\lim _{y \rightarrow-0} X=X^{+}-X^{-} .
$$

A partir de agora, como nos modelos Randall-Sundrum, vamos impor a simetria $\mathbb{Z}_{2}$ e fixar a brana num ponto fixo da dimensão extra. Com isso, a simetria imposta relaciona a curvatura extrínseca da brana com o seu tensor energia-momento

$$
K_{\mu \nu}^{+}=-K_{\mu \nu}^{-}=\frac{1}{2} \kappa_{5}\left(S_{\mu \nu}-\frac{1}{3} q_{\mu \nu} S\right) .
$$

Por causa dessa simetria, podemos calcular coisas em ambos os lados, + e -, da brana. Substituindo (3.52) em (3.44) obtemos

$$
{ }^{(4)} G_{\mu \nu}=-\Lambda_{4 D} q_{\mu \nu}+8 \pi G_{N} \tau_{\mu \nu}+\kappa_{5}^{2} \pi_{\mu \nu}-E_{\mu \nu}
$$

onde

$$
\begin{gathered}
\Lambda_{4 D}=\frac{1}{2} \kappa_{5}^{2}\left(\Lambda_{5 D}+\frac{1}{6} \kappa_{5}^{2} \lambda^{2}\right), \\
G_{N}=\frac{\kappa_{5}^{2} \lambda}{48 \pi}
\end{gathered}
$$


e

$$
\pi_{\mu \nu}=-\frac{1}{4} \tau_{\mu \alpha} \tau_{\nu}{ }^{\alpha}+\frac{1}{12} \tau \tau_{\mu \nu}+\frac{1}{8} q_{\mu \nu} \tau_{\alpha \beta} \tau^{\alpha \beta}-\frac{1}{24} q_{\mu \nu} \tau^{2} .
$$

As equações (3.53) são, finalmente, as equações de Einstein projetadas na brana e se tornam as conhecidas equações da Relatividade Geral quando tomamos o limite de $\kappa \rightarrow 0$, mantendo $G_{N}$ finito e desprezando $E_{\mu \nu}$. O tensor $E_{\mu \nu}$ é a parte do tensor 5-dimensional de Weyl projetado na brana e carrega consigo a informação do campo gravitacional fora da brana e como ele a influencia. Portanto, $E_{\mu \nu}$ depende da geometria do bulk e não pode ser determinado livremente pois é vinculado ao movimento da matéria na brana. Usando a identidade de Bianchi, $D^{\mu}{ }^{(4)} G_{\mu \nu}=0$, observamos a relação entre $E_{\mu \nu}$ e $\tau_{\mu \nu}$

$$
\begin{aligned}
D^{\mu} E_{\mu \nu} & =K^{\alpha \beta}\left(D_{\nu} K_{\alpha \beta}-D_{\beta} K_{\nu \alpha}\right), \\
& =\frac{1}{4} \kappa_{5}^{2}\left[\tau^{\alpha \beta}\left(D_{\nu} \tau_{\alpha \beta}-D_{\beta} \tau_{\nu \alpha}\right)+\frac{1}{3}\left(\tau_{\mu \nu}-q_{\mu \nu} \tau\right) D^{\mu} \tau\right] .
\end{aligned}
$$

Vemos, então, que $E_{\mu \nu}$ não é livremente determinado, mas sua divergência é vinculada à matéria na brana. Se decompusermos $E_{\mu \nu}$ em uma parte transversa, $E_{\mu \nu}^{T T}$, e numa parte longitudinal, $E_{\mu \nu}^{L}$, a última será determinada completamente pela matéria. Dessa forma, se $E_{\mu \nu}^{T T}$ for zero, as equações (3.53) tratarão somente de elementos contidos na brana pois a parte transversa corresponde a ondas gravitacionais no bulk. Assim, para resolvermos completamente as equações na brana deveremos, em geral, resolver as equações no bulk.

No próximo capítulo, veremos como caracterizar e construir, de forma geral, soluções de buracos negros esfericamente simétricos na brana. 


\section{Capítulo 4}

\section{Buracos Negros Estáticos com Simetria Esférica}

A idéia de um objeto tão compacto e com um campo gravitacional suficientemente intenso de tal forma que a própria luz não consiga escapar foi proposta originalmente por John Mitchel em 1783 [24]. O conceito de campo não foi usado a princípio, e toda argumentação foi embasada na Teoria da Gravitação Newtoniana. No século XX, a mesma idéia retornou com toda força depois da Teoria da Relatividade Geral e, devido a John Wheeler num artigo intitulado The Know and the Unknow, publicado em 1968 na American Scientist e na American Scholar, um objeto deste tipo recebeu o nome de buraco negro.

\section{$4.1 \quad$ Horizontes}

Podemos representar um espaço-tempo de forma muito útil através dos diagramas de Penrose, pois regiões infinitas podem ser representadas por linhas finitas e pontos. Nestes diagramas, de forma pouco rigorosa, o infinito corresponde ao destino e/ou origem de curvas do tipo luz, tempo e espaço. Então, temos três tipos de infinitos: tipo luz, tipo tempo e tipo espaço, que correspondem ao destino e/ou origem de curvas do tipo luz, tempo e espaço, respectivamente (veja por exemplo [21]). Exemplo de um diagrama de Penrose para o espaço-tempo Schwarzschild anti-de Sitter, conforme [22], é mostrado na figura 4.1 . 


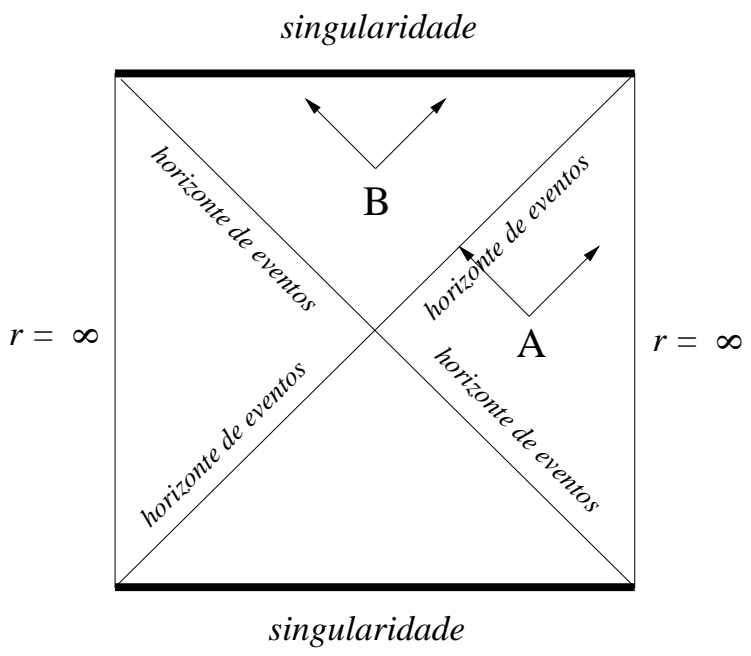

Figura 4.1: O diagrama de Penrose do espaço-tempo Schwarzschild anti-de Sitter. No ponto A, podemos enviar sinais de luz que terminarão no infinito, $r=\infty$, ou no horizonte de eventos. No ponto $B$, dentro do horizonte, estes sinais só poderão terminar na singularidade.

No espaço-tempo de Minkowski, curvas do tipo luz terminam no infinito tipo luz. Entretanto, um espaço-tempo que contém um buraco negro possui uma região onde curvas do tipo luz não terminam no infinito tipo luz, curvas do tipo luz terminam numa singularidade, conforme é indicado na figura 4.1. Assim, o contorno desta região é chamado horizonte de eventos.

Então, para definir um horizonte de eventos devemos conhecer a estrutura passada ou futura do infinito do tipo luz e, conseqüentemente, isso faz com que um horizonte de eventos seja um objeto não local. Ao contrário, um horizonte de Killing (veja [21]) é um objeto local, pois é definido como uma superfície $H$ onde os vetores de Killing $X^{\mu}$ tangentes à ela são do tipo luz quando calculados em $H$, ou seja, $\left.X^{\mu} X_{\mu}\right|_{H}=0$. Em [20] é mostrado que num espaço-tempo estacionário e assintoticamente plano o horizonte de eventos coincide com o horizonte de Killing.

Métricas estáticas em 4 dimensões com simetria esférica podem ser escritas na forma geral

$$
d s^{2}=A(r) d t^{2}-\frac{d r^{2}}{B(r)}-r^{2}\left(d \theta^{2}+\operatorname{sen}^{2} \theta d \phi^{2}\right) .
$$

A partir de um dado $A(r)$, construiremos soluções estáticas esfericamente simétricas através do cálculo de uma família de funções $B(r)$. Entretanto, antes de prosseguirmos, vamos formular as condições que a função $A(r)$ conduz a métricas de buracos negros com horizontes de Killing. Para tal feito, utilizaremos a coordenada quase global, $u$, 
que obedece a

$$
g_{t t} g_{u u}=-1
$$

O motivo que nos faz adotar tal coordenada reside no fato de que (4.1) toma a forma da métrica de Schwarzschild e assim podemos utilizar toda ferramenta (extensão analítica da métrica, por exemplo) utilizada nessa solução bem conhecida. Utilizando a coordenada $u,(4.1)$ fica

$$
d s^{2}=\mathcal{A}(u) d t^{2}-\frac{d u^{2}}{\mathcal{A}(u)}-r^{2}(u)\left(d \theta^{2}+\operatorname{sen}^{2} \theta d \phi\right),
$$

onde

$$
\mathcal{A}(u)=A(r), \quad r(u)=r \quad \text { e } \quad \mathcal{A}(u)\left(\frac{d r}{d u}\right)^{2}=B(r) .
$$

Nas geometrias em que $A(r)=B(r)$ (por exemplo, solução de Schwarzschild), temos que $u=r$, ou seja, $d r=d u$ a partir de (4.4).

Os zeros de $\mathcal{A}(u)$ representam pontos onde a métrica no sistema de coordenadas $(t, u, \theta, \phi)$ é problemática. Uma condição necessária para que (4.3) descreva um espaçotempo fisicamente razoável é que as funções $\mathcal{A}(u)$ e $r(u)$ sejam $C^{\infty}$ (ou pelo menos diferenciáveis um certo número de vezes). Isto implica que em um zero, $u=h$, da função $\mathcal{A}(u)$ devemos ter

$$
\mathcal{A}(u) \sim(u-h)^{k} \quad \text { e } \quad r(u) \approx r_{+}+\operatorname{cte}(u-h)^{s}
$$

onde $k$ é um inteiro positivo diferente de zero, $s$ também é um inteiro positivo diferente de zero, que caracteriza o comportamento de $r(u), r_{+}$é o raio do horizonte de eventos e o símbolo $\sim$ relaciona grandezas de mesma magnitude e ordem dentro de um certo limite. Definimos a ordem do horizonte pelo número $k$. Quando $k=1$, dizemos que o horizonte é simples; $k=2$ denota um horizonte duplo e assim por diante.

A seguir, vamos mostrar que a superfície dada por $u=h$ é um horizonte de Killing. Como foi visto no capítulo 2, se uma métrica é independente de uma coordenada, o espaço-tempo descrito por ela tem um campo vetorial de Killing. A métrica (4.3) é independente da coordenada $t$, logo podemos escrever um campo vetorial de Killing como

$$
X=\frac{\partial}{\partial t}
$$


ou expresso através de suas coordenadas como $X^{\mu}=(1,0,0,0)$, que satisfaz a condição

$$
\mathcal{L}_{X} g_{\mu \nu}=0 .
$$

A norma de um vetor desse campo é dada por

$$
X^{2}=g_{00} X^{0} X^{0}=\mathcal{A}(u) .
$$

No horizonte de eventos, temos que $\mathcal{A}(h)=0$ e esse vetor é do tipo luz pois sua norma é nula. Então, o campo vetorial dado por $\partial / \partial t$ é um campo de Killing do tipo luz e a superfície em $u=h$ é um horizonte de Killing, uma membrana de caminho único. Podemos, igualmente, mostrar que a métrica escrita na forma (4.1) tem um horizonte de Killing em $r=r_{+}$, onde $r_{+}$é raiz de $A(r)$. Um campo vetorial de Killing pode ser escrito como (4.6), sendo suas coordenadas dadas por $X^{\mu}=(1,0,0,0)$, e sua norma dada por

$$
X^{2}=g_{00} X^{0} X^{0}=A(r) .
$$

Em $r=r_{+}$, temos vetores do tipo luz e, dessa forma, a superfície em $r_{+}$é um horizonte de Killing.

Como pode ser visto por exemplo em [28], a definição de gravidade superficial, $\kappa_{+}$, em termos de um campo de Killing, $X$, é

$$
\nabla\left(X^{\mu} X_{\mu}\right)=2 \kappa_{+} X .
$$

Tomando a derivada de Lie de $\kappa_{+}$em relação a $X$ obtem-se

$$
\mathcal{L}_{X} \kappa_{+}=0,
$$

ou seja, $\kappa_{+}$é uma constante de movimento e, além disso, é constante no horizonte de eventos. Utilizando as coordenadas quase globais e um campo de Killing do tipo $X^{\mu}=(1,0,0,0)$, com auxílio de (4.8), (4.10) fica

$$
\kappa_{+}=\left.\frac{1}{2} \frac{d \mathcal{A}(u)}{d u}\right|_{u=h} .
$$

A gravidade superficial, $\kappa_{+}$, tem uma analogia com um conceito da termodinâmica, a temperatura. Na termodinâmica de buracos negros, $\kappa_{+}$faz o papel de temperatura e, assim como na termodinâmica, a lei zero diz que a temeperatura, $\kappa_{+}$, é uniforme sobre um corpo em equilíbrio, ou seja, um buraco negro estacionário. 


\subsection{Extensão da métrica}

Veremos, a seguir, como obter a coordenada tartaruga, $\tilde{r}^{*}$, a partir da coordenada $u$, e estender analiticamente o espaço-tempo. A coordenada tartaruga em termos da coordenada $r$ é

$$
\frac{d \tilde{r}^{*}}{d r}=\frac{1}{h(r)}
$$

sendo

$$
h(r)=\sqrt{A(r) B(r)} .
$$

Com auxílio de (4.4), podemos escrever

$$
d r=\sqrt{\frac{A(r)}{B(r)}} d u
$$

e usar a relação acima em (4.13) para obter

$$
\frac{d \tilde{r}^{*}}{d u}=\frac{1}{\mathcal{A}(u)}
$$

Podemos utilizar (4.15), com auxílio de (4.14) e $A(r)=\mathcal{A}(u)$, na equação (4.12) para obter

$$
\kappa_{+}=\left.\frac{1}{2} \frac{d h(r)}{d r}\right|_{r=r_{+}} .
$$

Definiremos, então, a coordenada avançada $v$

$$
v=t+\tilde{r}^{*}
$$

onde neste novo sistema de coordenadas a métrica (4.3), utilizando (4.16), fica

$$
d s^{2}=\mathcal{A}(u) d v^{2}-2 d v d u-r(u)^{2}\left(d \theta^{2}-\operatorname{sen}^{2} \theta d \phi^{2}\right)
$$

e a coordenada retardada

$$
w=t-\tilde{r}^{*}
$$

onde neste novo sistema de coordenadas a métrica (4.3), utilizando (4.16), fica

$$
d s^{2}=\mathcal{A}(u) d w^{2}+2 d w d u-r(u)^{2}\left(d \theta^{2}-\operatorname{sen}^{2} \theta d \phi^{2}\right) .
$$

A métrica, escrita nas formas (4.19) ou (4.21), é completamente regular no horizonte, 
onde $\mathcal{A}=0$. Dessa forma, a extensão da métrica para além do horizonte pode ser realizada, redefinindo os limites da coordenada $u$ de forma conveniente. Em princípio, o processo pode ser repetido mais vezes se necessário, até obtermos a extensão maximal do espaço-tempo.

O comportamento da coordenada tartaruga, $\tilde{r}^{*}(r)$, para valores grandes de $r$, como pode ser visto na figura 4.2, indica se a geometria estudada é assintoticamente plana, de Sitter ou anti-de Sitter, conforme (2.37), (2.38) e (2.39). Como $d \tilde{r}^{*} / d r$ é dada pelo inverso da função $h(r)$, no caso de uma geometria assintoticamente plana

$$
\lim _{r \rightarrow \infty} \frac{1}{h(r)}=1
$$

e a curva $\tilde{r}^{*}(r)$ tende a uma reta com inclinação de 45 graus em relação ao eixo $r$. No caso de uma geometria assintoticamente de Sitter, $\tilde{r}^{*}(r)$ não está definida para valores de $r$ maiores que um certo valor crítico, $r_{C}$. Quando tomamos o limite para $r \rightarrow r_{C}$ obtemos

$$
\lim _{r \rightarrow r_{C}} \frac{1}{h(r)} \rightarrow \infty
$$

e o gráfico de $\tilde{r}^{*}(r)$ mostra que esta função diverge quando $r=r_{C}$. Para o caso de uma geometria assintoticamente anti-de Sitter, $\tilde{r}^{*}(r)$ tende a um valor constante quando $r$ cresce, como conseqüência de

$$
\lim _{r \rightarrow \infty} \frac{1}{h(r)}=0
$$

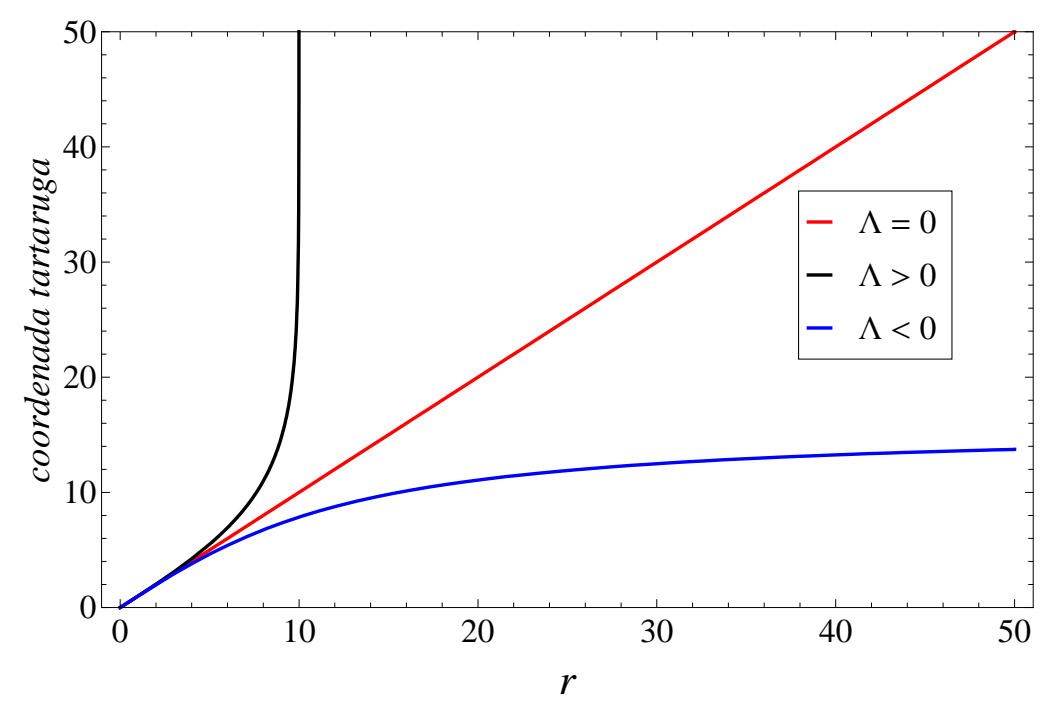

Figura 4.2: O comportamento assintótico da coordenada tartaruga, $\tilde{r}^{*}$, é mostrado para as geometrias assintoticamente plana, $\Lambda=0$, de Sitter, $\Lambda>0$, e anti-de Sitter, $\Lambda<0$. 


\subsection{Construção de soluções}

A partir da função $A(r)$, podemos obter uma família de funções $B(r)$ e cada membro desta família corresponderá a uma métrica do tipo (4.1) diferente. Nesta seção, descreveremos o procedimento para obtenção dessas soluções.

Podemos escrever os escalares de Ricci, $R$, e o de Kretschmann, $\kappa$, usando (4.1), da seguinte forma

$$
R=\frac{2}{r^{2}}(1-B)-B\left[\frac{A_{r r}}{A}-\frac{A_{r}^{2}}{2 A^{2}}+\frac{A_{r} B_{r}}{2 A B}+\frac{2}{r}\left(\frac{A_{r}}{A}+\frac{B_{r}}{B}\right)\right]
$$

e

$$
\kappa=R_{\mu \nu}^{\rho \sigma} R_{\rho \sigma}^{\mu \nu}=4 K_{1}^{2}+8 K_{2}^{2}+8 K_{3}^{2}+4 K_{4}^{2}
$$

onde

$$
\begin{gathered}
K_{1}=\frac{B}{4}\left(\frac{2 A A_{r r}-A_{r}^{2}}{A^{2}}+\frac{A_{r} B_{r}}{A B}\right), \\
K_{2}=\frac{B A_{r}}{2 r A}, \quad K_{3}=\frac{B_{r}}{2 r}, \quad K_{4}=\frac{1-B}{r^{2}} .
\end{gathered}
$$

O símbolo subscrito $r$ significa $d / d r$. A finitude de $\kappa$ é um critério de regularidade para geometrias baseadas em (4.1), pois este escalar é a soma das componentes do tensor de curvatura.

Impondo que o lado direito de (4.25) seja uma função de $r, R(r)$, e definindo

$$
f(r)=r B(r)
$$

obtemos uma equação diferencial ordinária de primeira ordem

$$
f_{r}(r)+\frac{r\left(2 A(r) A_{r r}(r)-A_{r}^{2}\right)+3 A(r) A_{r}(r)}{A(r) g(r)} f(r)=\frac{2 A(r) Q(r)}{g(r)},
$$

cuja solução geral é (veja [5])

$$
f(r)=\frac{2 A(r) e^{3 \Gamma}}{g(r)^{2}}\left[\int_{r_{+}}^{r} g(r) Q(r) e^{-3 \Gamma} d r+C\right],
$$

onde

$$
g(r)=4 A(r)+r A_{r}(r)
$$




$$
\Gamma=\int_{r_{+}}^{r} \frac{A_{r}(r)}{g(r)} d r
$$

$\mathrm{e}$

$$
Q(r)=2-r^{2} R(r) .
$$

Dessa forma, escolhendo um $A(r)$ liso e impondo um $R(r)$, calculamos $f(r)$ por (4.29) e obtemos imediatamente uma família de funções $B(r)$ com o auxílio de (4.27). A métrica ficará completamente conhecida desde que a constante de integração $C$, proveniente de (4.29), seja fixada, ou seja, um membro da famíla de funções $B(r)$ seja escolhido.

Em universos brana, pode-se obter soluções das equações de Einstein projetadas na brana sem especificar o tensor $E_{\mu \nu}$. Não discutiremos as propriedades do bulk, a existência de soluções neste espaço-tempo 5-dimensional é garantida por teoremas de imersão [23]. A partir das equações projetadas obtidas no capítulo 3

$$
G^{\nu}{ }_{\mu}=-\Lambda_{4 D} q_{\mu}^{\nu}+8 \pi G_{N} \tau_{\mu}^{\nu}+\kappa_{5}^{2} \pi_{\mu}^{\nu}-E^{\nu}{ }_{\mu},
$$

onde $\Lambda_{4 D}, q_{\mu \nu}$ e $\tau_{\mu}^{\nu}$ são a constante cosmológica, a métrica e o tensor energia-momento na brana, respectivamente, $G_{N}$ é a constante gravitacional, $\kappa_{5}$ é constante gravitacional em 5 dimensões e $\pi^{\nu}{ }_{\mu}$ é dado por

$$
\pi_{\mu}^{\nu}=-\frac{1}{4} \tau_{\mu \alpha} \tau_{\nu}^{\alpha}+\frac{1}{12} \tau \tau_{\mu \nu}+\frac{1}{8} q_{\mu \nu} \tau_{\alpha \beta} \tau^{\alpha \beta}-\frac{1}{24} q_{\mu \nu} \tau^{2},
$$

sabendo que $\tau=\tau_{\mu}^{\mu}$, o traço de $E^{\nu}{ }_{\mu}$ é nulo, ou seja, $E^{\mu}{ }_{\mu}=0$ e que em 4 dimensões $G^{\nu}{ }_{\mu}$ é dado por

$$
G^{\nu}{ }_{\mu}=R^{\nu}{ }_{\mu}-\frac{1}{2} q_{\nu}^{\mu} R,
$$

podemos igualar (4.33) a (4.35), fazendo $\nu=\mu$, e obter

$$
R=4 \Lambda_{4 D}-8 \pi G_{N} \tau+\kappa_{5}^{4} \pi_{\mu}^{\mu} .
$$

Conseqüentemente, vemos que o escalar de Ricci $R$ dependerá de variáveis definidas na brana, pois a constante gravitacional em 5 dimensões é dada por

$$
\kappa_{5}^{2}=\frac{48 \pi^{2} G_{N}}{\lambda},
$$

sendo $\lambda$ a tensão na brana.

Em [5] é mostrado como a escolha do valor da constante de integração de (4.29) conduz a soluções diferentes de buracos negros estáticos e esfericamente simétricos na 
brana. Para funções $A(r)$ que podem ser escritas próximas ao horizonte na forma $A(r) \sim\left(r-r_{+}\right)^{1 / s}$, onde $s \in \mathbb{N}$, e (4.30) seja maior do que zero na região de interesse, um buraco negro terá um horizonte simples se a constante de integração for maior do que zero e terá um horizonte de ordem $2+p s$, onde $p$ é o expoente da aproximação de $Q(r)=2-r^{2} R(r) \sim\left(r-r_{+}\right)^{p}$ entorno do horizonte, caso a constante de integração seja nula. Para funções $A(r)$ que podem ser escritas próximas ao horionte como $A(r) \sim$ $\left(r-r_{+}\right)^{2 / s}$, onde $s \in \mathbb{N}$, e (4.30) seja maior do que zero na região de interesse, um buraco negro terá um um horizonte de ordem $2+p s$ caso a constante de integração seja nula e a função $Q(r)$ se comporte como $\left(r-r_{+}\right)^{p}$ próxima ao horizonte. Neste caso, está excluída uma constante de integração diferente de zero.

Podemos encontrar as componentes do tensor energia-momento efetivo, $\tau^{\mu \nu e f}$, na brana se escrevermos (3.53) como

$$
G_{\mu}^{\nu}+\delta_{\mu}^{\nu} \Lambda_{4 D}=\kappa_{4}^{2} \tau_{\mu}^{\nu} \text { ef }
$$

sendo $\kappa_{4}^{2}=8 \pi G_{N}=8 \pi$, de acordo com as unidades geométricas. Então, a densidade, $\rho^{e f}$, pressão radial, $p_{\text {rad }}^{e f}$, e a pressão tangencial, $p_{\perp}^{e f}$, efetivas são expressas em termos das funções $A(r)$ e $B(r)$ do seguinte modo

$$
\begin{gathered}
G^{t}{ }_{t}=-4 \Lambda_{4 D}-\kappa_{4}^{2} \rho^{e f}=\frac{B-1}{r^{2}}+\frac{B_{r}}{r} \\
G^{r}{ }_{r}=-4 \Lambda_{4 D}+\kappa_{4}^{2} p_{r a d}^{e f}=\frac{B-1}{r^{2}}+\frac{B A_{r}}{A r} \\
G^{\theta}{ }_{\theta}=G_{\phi}^{\phi}=-4 \Lambda_{4 D}+\kappa_{4}^{2} p_{\perp}^{e f}=\frac{B}{4}\left[\frac{2 A_{r r}}{A}-\frac{A_{r}^{2}}{A^{2}}+\frac{A_{r} B_{r}}{A B}+\frac{2}{r}\left(\frac{A_{r}}{A}+\frac{B_{r}}{B}\right)\right] .
\end{gathered}
$$

Numa brana onde há vácuo, $\tau^{\mu \nu}=0$ mas $\tau^{\mu \nu e f}$ pode não ser nulo, pois o tensor energiamomento efetivo contém a influência do bulk na brana.

No próximo capítulo, utilizaremos o procedimento descrito anteriormente para obter métricas de buracos negros estáticos e esfericamente simétricos com constante cosmológica diferente de zero na brana. 


\section{Capítulo 5}

\section{Soluções com constante cosmológica}

A introdução de uma constante cosmológica nas equações de Einstein muda drasticamente o caráter assintótico das soluções esfericamente simétricas. Dependendo do sinal da constante cosmológica, temos uma geometria assintoticamente de Sitter ou anti-de Sitter, caso o sinal seja positivo ou negativo, respectivamente. Casos com a constante cosmológica nula já foram estudados por [5, 12].

\subsection{Soluções assintoticamente de Sitter}

\subsubsection{Raízes de $A(r)$ e $g(r)$}

A partir de um $A(r)$ do tipo Schwarzschild-de Sitter

$$
A(r)=1-\frac{2 m}{r}-\frac{r^{2}}{a^{2}}
$$

onde $m$ é a massa e $a^{2}=12 / R$, sendo $R$ o escalar de Ricci, utilizamos o procedimento descrito no capítulo anterior para a obtenção de uma família de funções $B(r)$ na brana sem conteúdo de matéria. Podemos escrever (5.1) como

$$
A(r)=\frac{-r^{3}+a^{2} r-2 m a^{2}}{a^{2} r}
$$

e definir um polinômio para estudar as raízes de $A(r)$. Assim, se escolhermos

$$
p(r)=-r^{3}+a^{2} r-2 m a^{2},
$$


então $p(r)$, ou seja, $A(r)$ terá um máximo em $r=a / \sqrt{3}$ e um mínimo em $r=-a / \sqrt{3}$. Queremos que $A(r)$ tenha 3 raízes reais, e para que isso ocorra

$$
p\left(\frac{a}{\sqrt{3}}\right)=\frac{2 a^{3}}{3 \sqrt{3}}+2 m a^{2}>0
$$

e

$$
p\left(-\frac{a}{\sqrt{3}}\right)=-\frac{2 a^{3}}{3 \sqrt{3}}+2 m a^{2}<0 .
$$

Podemos ilustrar essa situação através do gráfico abaixo.

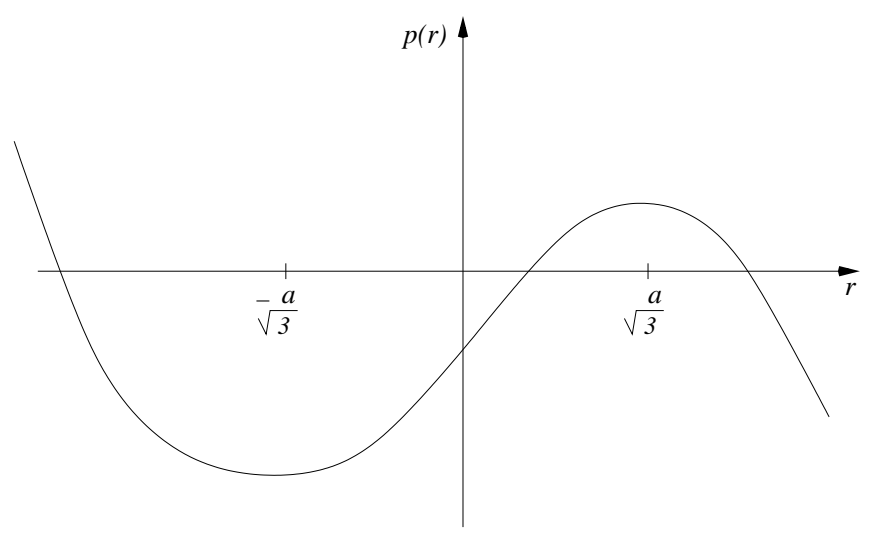

Figura 5.1: Gráfico de $p(r)$ com duas raízes positivas e uma negativa. Os valores $r=a / \sqrt{3}$ e $r=-a / \sqrt{3}$ indicam um máximo e um mínimo, respectivamente.

Dessa forma, $p(r)$ terá três raízes reais quando (5.4) e (5.5) forem satisfeitas simultaneamente. O valor de $a$ que satisfaz ambas condições é $a>3 \sqrt{3} m$, e como $p(0)=-2 m a^{2}<0$ e os valores de máximo e mínimo são eqüidistantes da origem, teremos duas raízes positivas, $r_{+}$e $r_{c}$, e uma raiz negativa, $r_{n}$, como ilustrado no gráfico 5.1. As raízes positivas têm um signficado físico; $r_{+}$é o raio do horizonte de eventos e $r_{c}$ é o raio do horizonte cosmológico. Ambas são dadas por

$$
r_{+}=\frac{1}{2}\left[\frac{a^{2}+\mathcal{F}(m, a)^{\frac{2}{3}}-i \sqrt{3}\left(a^{2}-\mathcal{F}(m, a)^{\frac{2}{3}}\right)}{\sqrt{3}(\mathcal{F}(m, a))^{\frac{1}{3}}}\right]
$$

e

$$
r_{c}=\frac{1}{2}\left[\frac{a^{2}+\mathcal{F}(m, a)^{\frac{2}{3}}+i \sqrt{3}\left(a^{2}-\mathcal{F}(m, a)^{\frac{2}{3}}\right)}{\sqrt{3} \mathcal{F}(m, a)^{\frac{1}{3}}}\right],
$$

onde

$$
\mathcal{F}(m, a)=a^{2}\left(3 \sqrt{3} m+\sqrt{27 m^{2}-a^{2}}\right) .
$$


Para obter uma família de funções $B(r)$, a partir do procedimento descrito no capítulo anterior, a função $g(r)=4 A(r)+4 A_{r}(r)$ não deve ter zeros no intervalo de interesse estudado, $r_{+} \leq r \leq r_{c}$, pois $g(r)$ aparece no numerador em (4.29). A seguir, vamos mostrar que $g(r)$ no caso Schwarzschild-de Sitter sempre tem para $a>3 \sqrt{3} m$ uma raiz, $r_{g}$, no intervalo de interesse. A função $g(r)$ para esse caso é

$$
g(r)=4-\frac{6 m}{r}-\frac{6 r^{2}}{a^{2}},
$$

e para estudar os seus zeros vamos reescrevê-la como

$$
g(r)=\frac{6 r^{3}+4 a^{2} r-6 m a^{2}}{a^{2} r},
$$

e determinar os zeros do polinômio

$$
q(r)=6 r^{3}+4 a^{2} r-6 m a^{2} .
$$

Para valores de $a>3 \sqrt{3} m$, o polinômio $q(r)$ terá três raízes reais, como pode ser visto a partir das condições

$$
q\left(\frac{2 a}{3 \sqrt{3}}\right)=\frac{56 a^{3}}{27 \sqrt{3}}-6 m a^{2}>0
$$

e

$$
q\left(-\frac{2 a}{3 \sqrt{3}}\right)=-\frac{56 a^{3}}{27 \sqrt{3}}-6 m a^{2}<0,
$$

sendo $r=2 a / 3 \sqrt{3}$ e $r=-2 a / 3 \sqrt{3}$ pontos de máximo e mínimo, respectivamente, de $q(r)$. As condições (5.12) e (5.13) serão satisfeitas para $a>81 \sqrt{3} m / 28$. Como queremos três raízes reais para $A(r)$, e para que isso ocorra $a$ deve ser maior do que $3 \sqrt{3} m$, o valor de $a$ que satisfaz as condições (5.12) e (5.13) não é incompatível com $3 \sqrt{3} m$. Assim, $a>3 \sqrt{3} m$ é compatível com as condições que envolvem $p(r)$ e $q(r)$. O polinômio $q(r)$ tem duas raízes para $r$ positivo, pois $q(0)=-6 m a^{2}$ e os pontos de máximo e mínimo são eqüidistantes da origem. Logo em seguida, vamos mostrar que $g(r)$ tem uma raiz entre as raízes positivas de $A(r)$ definindo o polinômio

$$
d(r)=p(r)-q(r)
$$

que terá zeros onde $A(r)=g(r)$. O polinômio $d(r)$ tem dois zeros para $r$ positivo quando $a>2 \sqrt{5} m$, ou seja, não é incompatível com o valor adotado anteriormente, $a>3 \sqrt{3} m$. $\mathrm{O}$ fato de $d(r)$ ter dois zeros para valores positivos de $r, q(2 a / 3 \sqrt{3})>p(a / \sqrt{3})$ e $q(a / \sqrt{3})>0$ garante que $q(r)$ tenha uma raiz entre as duas raízes positivas de $p(r)$, ou 
seja, $g(r)$ tem uma raiz, $r_{g}$, entre $\left[r_{+}, r_{c}\right]$. Conseqüentemente, não existe um valor de $a$ tal que $g(r)$ tenha um zero fora do nosso intervalo de interesse e, devido a esse fato, o uso de (4.28) é problemático. A figura número 5.2 ilustra o que foi dito.

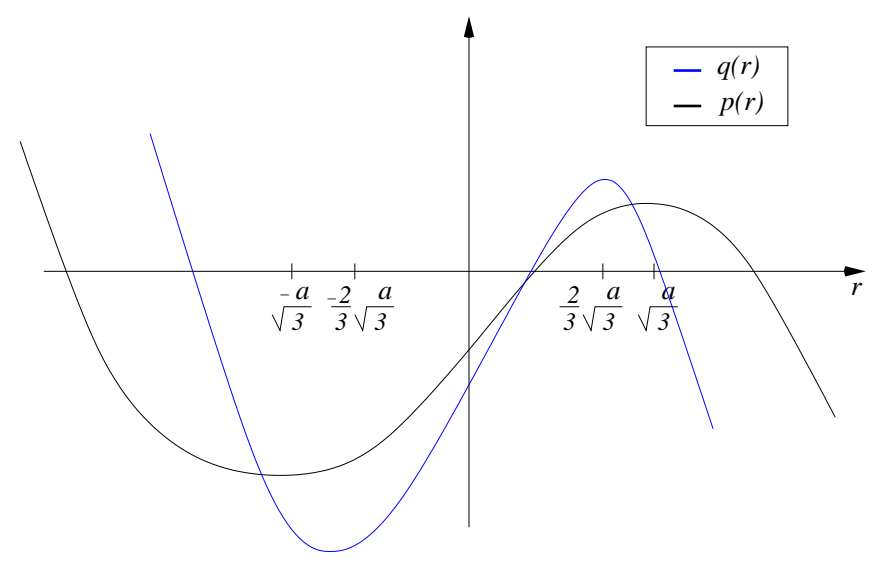

Figura 5.2: Se $A(r)$ tiver 3 raízes reais, sendo duas positivas e uma negativa, a deve ser maior do que $3 \sqrt{3} m$ e, consequentemente, $g(r)$, ou seja $q(r)$, terá pelo menos uma raíz entre as duas raízes positivas de $A(r)$ ou $p(r)$.

Entretanto, para $a=3 \sqrt{3} m$ as funções $A(r)$ e $g(r)$ terão apenas uma raiz positiva e o valor desta raiz será o mesmo para ambas funções. Este resultado pode ser mostrado se escrevermos $A(r)$ na forma

$$
A(r)=\left(r-r_{+}\right)\left(r-r_{c}\right) K(r),
$$

onde $K(r)$ não tem zeros em $r_{+}$e $r_{c}$. Com $r_{+}=r_{c}, A(r)$ e $g(r)$ ficam

$$
\begin{aligned}
A(r) & =\left(r-r_{+}\right)^{2} K(r) \\
g(r) & =4\left(r-r_{+}\right)^{2} K(r)+r\left[2\left(r-r_{+}\right) K(r)+\left(r-r_{+}\right)^{2} K_{r}(r)\right],
\end{aligned}
$$

onde subscrito $r$ indica a derivada em relação a $r$. Dessa forma, $A(r)$ e $g(r)$ terão zeros em $r_{+}$e o intervalo de interesse agora é $r_{+}<r<\infty$. Assim, podemos utilizar o procedimento descrito no capítulo anterior e obter um resultado para (4.29) dentro desse intervalo. 


\subsubsection{Família de Soluções}

A função $\Gamma(r)$, dada por (4.31), para este caso é

$$
\begin{aligned}
\Gamma(r) & =\frac{1}{3} \ln \left[\frac{3 m}{r}\right]+\frac{1}{3 \sqrt{5}} \ln \left[\frac{1-\frac{r}{m}+\left(1-\frac{r}{3 m}\right) \sqrt{5}}{1-\frac{r}{m}-\left(1-\frac{r}{3 m}\right) \sqrt{5}}\right] \\
& +\frac{1}{3} \ln \left[-1+\frac{r}{3 m}+\frac{r^{2}}{9 m^{2}}\right]
\end{aligned}
$$

sendo $r_{+}=3 m$ com a escolha $a=3 \sqrt{3} \mathrm{~m}$. Obtivemos, em seguida, a função $B(r)$ impondo $R=4 \Lambda_{4 D}=12 / a^{2}$, de acordo com (4.36), com auxílio de (4.29) e (4.27). Com isso, a família de funções $B(r)$ obtida é

$$
\begin{aligned}
B(r)= & -\frac{C m(r+6 m)}{2 r}\left[\left(\frac{7+3 \sqrt{5}}{2}\right)^{\frac{1}{\sqrt{5}}}\left(r-r_{1}\right)^{\frac{1}{\sqrt{5}}-1}\left(r+r_{2}\right)^{\frac{-1}{\sqrt{5}}}\left(r-r_{2}\right)^{-1}\right]+ \\
& +\left(1-\frac{2 m}{r}-\frac{r^{2}}{27 m^{2}}\right)
\end{aligned}
$$

onde $r_{1}=3 m(\sqrt{5}-1) / 2$ e $r_{2}=-3 m(\sqrt{5}+1) / 2$, somente definida no intervalo de interesse $r_{+}<r<\infty$ quando a constante de integração, $C$, for nula. Para o caso que $C \neq 0$, a função $B(r)$ não está definida dentro do intervalo escolhido e para $C=0$, temos, de acordo com (5.1), $A(r)=B(r)$, ou seja, a métrica Schwarzschild-de Sitter extrema (quando $r_{+}=r_{c}$, veja [13]) já conhecida

$$
d s^{2}=\left(1-\frac{2 m}{r}-\frac{r^{2}}{27 m^{2}}\right) d t^{2}-\left(1-\frac{2 m}{r}-\frac{r^{2}}{27 m^{2}}\right)^{-1} d r^{2}-r^{2} d \theta^{2}-r^{2} \operatorname{sen}^{2} \theta d \phi^{2} .
$$

Então, a família de funções $B(r)$ obtida só contém um membro, quando $C=0$.

\subsection{Soluções assintoticamente anti-de Sitter}

\subsubsection{Raízes de $A(r)$ e $g(r)$}

A partir de um $A(r)$ do tipo Schwarzschild anti-de Sitter

$$
A(r)=1-\frac{2 m}{r}+\frac{r^{2}}{a^{2}}
$$


onde, novamente, $m$ é a massa e $a^{2}=-12 / R$, sendo $R$ o escalar de Ricci, utilizamos o procedimento descrito no capítulo anterior para obtenção de uma família de funções $B(r)$ na brana sem conteúdo de matéria. A função $A(r)$ pode ser escrita como

$$
A(r)=\frac{r^{3}+a^{2} r-2 m a^{2}}{a^{2} r} .
$$

Podemos estudar seus zeros, máximos e mínimos definindo o polinômio

$$
t(r)=r^{3}+a^{2} r-2 m a^{2},
$$

e calculando sua derivada em relação a $r$

$$
t_{r}(r)=3 r^{2}+a^{2} .
$$

A equação (5.23) revela que (5.22) não tem máximos ou mínimos. Neste caso, $A(r)$ tem 3 raízes: 1 raiz real positiva, que representa o raio do horizonte de eventos

$$
r_{+}=\frac{\mathcal{G}(m, a)^{\frac{2}{3}}-a^{2}}{\sqrt{3} \mathcal{G}(m, a)^{\frac{1}{3}}}
$$

onde

$$
\mathcal{G}(m, a)=a^{2}\left(3 \sqrt{3} m+\sqrt{27 m^{2}+a^{2}}\right),
$$

e duas raízes complexas conjugadas. No caso Schwarzschild anti-de Sitter, o nosso intervalo de interesse será $r_{+} \leq r<\infty$.

A função $g(r)=4 A(r)+r A_{r}(r)$ neste caso fica

$$
g(r)=4-\frac{6 m}{r}+\frac{6 r^{2}}{a^{2}}
$$

$\mathrm{ou}$

$$
g(r)=\frac{6 r^{3}+4 a^{2} r-6 m a^{2}}{a^{2} r},
$$

e podemos igualmente definir um polinômio para estudar suas raízes. O polinômio

$$
v(r)=6 r^{3}+4 a^{2} r-6 m a^{2}
$$

e sua derivada em relação a $r$

$$
v_{r}(r)=18 r^{2}+4 a^{2}
$$


serão usados para estudar os zeros da função $g(r)$. Igualmente à função $A(r), g(r)$ não tem máximos ou mínimos, tem uma raiz real positiva

$$
r_{g}=\frac{4 a^{2}-2^{\frac{1}{3}} \mathcal{H}(m, a)^{\frac{2}{3}}}{3(2)^{\frac{2}{3}} \mathcal{H}(m, a)^{\frac{1}{3}}}
$$

onde

$$
\mathcal{H}(m, a)=a^{2}\left(\sqrt{32 a^{2}+729 m^{2}}-27 m\right),
$$

e duas raízes complexas conjugadas. Como $g(r)=3 A(r)+1+r^{2} / a^{2}$, de acordo com (5.20) e (5.26), $g(r)>A(r)$ e nos conduz a $r_{g}<r_{+}$. Dessa forma, não teremos problema ao usar o procedimento descrito no capítulo anterior e obter um resultado para (4.29) dentro do intervalo $r_{+} \leq r<\infty$. A figura 5.3 ilustra o comportamento das funções $A(r)$ e $g(r)$.

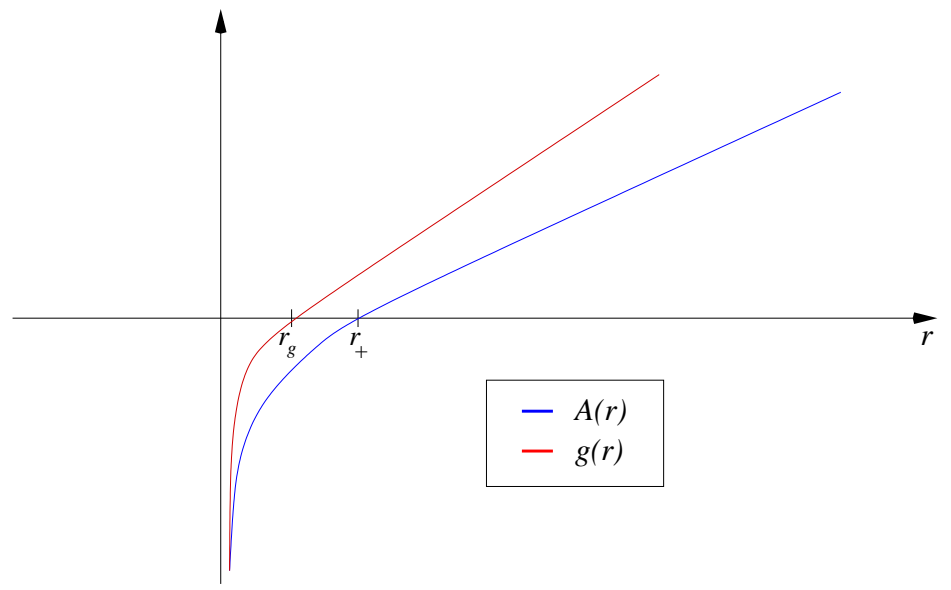

Figura 5.3: A função $g(r)$ não tem um zero no intervalo de interesse $r_{+} \leq r<\infty$.

\subsubsection{Família de Soluções}

A função $\Gamma(r)$, dada por (4.31), para este caso é

$$
\begin{aligned}
\Gamma(r) & =\frac{\left(2 k_{1}-c_{1} k_{2}\right)}{\sqrt{4 c_{2}-c_{1}^{2}}}\left[\operatorname{arctg}\left(\frac{2 r+c_{1}}{\sqrt{4 c_{2}-c_{1}^{2}}}\right)-\operatorname{arctg}\left(\frac{2 r_{+}+c_{1}}{\sqrt{4 c_{2}-c_{1}^{2}}}\right)\right] \\
& +k_{3} \ln \left[\frac{r}{r_{+}}\right]+k_{4} \ln \left[\frac{\left(r-c_{3}\right)}{\left(r_{+}-c_{3}\right)}\right]+\frac{1}{2} k_{2} \ln \left[\frac{r^{2}+c_{1} r+c_{2}}{r_{+}^{2}+c_{1} r_{+}+c_{2}}\right],
\end{aligned}
$$

onde $k_{1}, k_{2}, k_{3}, k_{4}, c_{1}, c_{2}$ e $c_{3}$ são constantes que dependem de $m$ e $a$. Por simplici- 
dade, omitimos as expressões explícitas destas constantes em termos dos parâmetros da métrica. Impondo $R=4 \Lambda_{4 D}=-12 / a^{2}$, de acordo com (4.36), com auxílio de (4.29) e (4.27), a família de funções $B(r)$ nesse caso só foi obtida numericamente. O gráfico de $B(r)$ para vários valores da constante de integração de (4.29) é exibido logo abaixo.

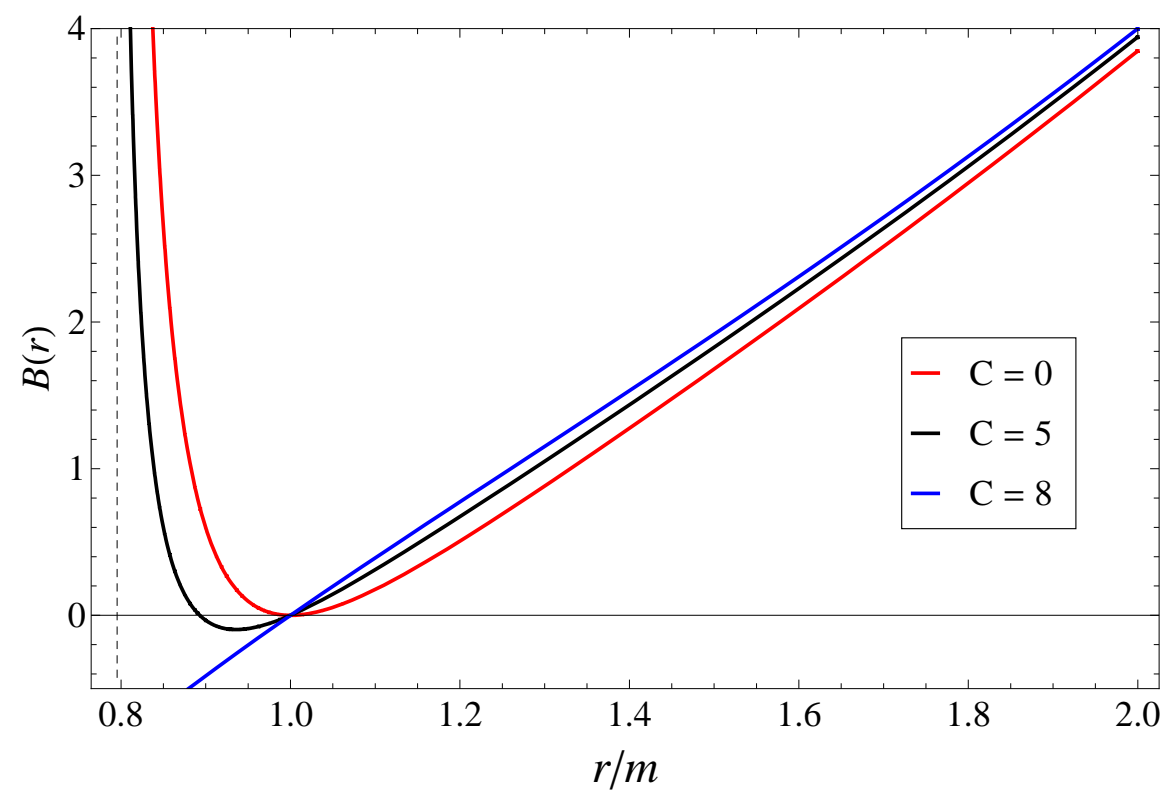

Figura 5.4: Gráfico da função $B(r)$ para vários valores de $C$ com $m=a=1$. O raio do horizonte de eventos e a raiz de $g(r)$ são, com estas escolhas, $r_{+}=1$ e $r_{g} \approx 0,8$, respectivamente. A curva azul representa o caso em que $A(r)=B(r)$. As funções $B(r)$, nos casos onde $A(r) \neq B(r)$, divergem quando $r \rightarrow r_{g}$, que é representada pela linha tracejada.

O gráfico de $B(r)$ mostra que para valores de $C$ que tornam $A(r) \neq B(r)$, a função $B(r)$ diverge quando $r \rightarrow r_{g}$. Isto pode ser mostrado se $A(r), g(r), Q(r)=2-r^{2} R$, $e^{3 \Gamma(r)}$ e $e^{-3 \Gamma(r)}$ forem escritos em torno de $r_{g}$ como

$$
\begin{gathered}
A(r)=A\left(r_{g}\right)+\mathcal{O}\left[\left(r-r_{g}\right)\right], \\
g(r)=G\left(r_{g}\right)\left(r-r_{g}\right)+\mathcal{O}\left[\left(r-r_{g}\right)^{2}\right], \\
Q(r)=Q\left(r_{g}\right)+\mathcal{O}\left[\left(r-r_{g}\right)\right] \\
e^{3 \Gamma(r)}=e^{3 \Gamma\left(r_{g}\right)}+\mathcal{O}\left[\left(r-r_{g}\right)\right]
\end{gathered}
$$

e

$$
e^{-3 \Gamma(r)}=e^{-3 \Gamma\left(r_{g}\right)}+\mathcal{O}\left[\left(r-r_{g}\right)\right]
$$


Substituindo as expansões acima na expressão para $f(r)$, obtemos

$$
\begin{aligned}
f(r) & =\frac{2 A(r) e^{3 \Gamma}}{g(r)^{2}}\left[\int_{r_{+}}^{r} g(r) e^{-3 \Gamma} Q(r) d r+C\right] \\
& =A\left(r_{g}\right)\left[2 e^{3 \Gamma\left(r_{+}\right)} C-Q\left(r_{g}\right)\left(r_{+}-r_{g}\right)^{2}\right]\left(r-r_{g}\right)^{-2}+\mathcal{O}\left[\left(r-r_{g}\right)^{-1}\right]
\end{aligned}
$$

Como $e^{3 \Gamma\left(r_{g}\right)}=0, f(r)=r B(r)$ e $1 / r=1 / r_{g}+\mathcal{O}\left[\left(r-r_{g}\right)\right]$, chegamos à expressão para a função $B(r)$ próxima a $r_{g}$

$$
B(r)=\frac{A\left(r_{g}\right)}{r_{g}}\left[-Q\left(r_{g}\right)\left(r_{+}-r_{g}\right)^{2}\right]\left(r-r_{g}\right)^{-2}+\mathcal{O}\left[\left(r-r_{g}\right)^{-1}\right] .
$$

Sendo $A\left(r_{g}\right)<0$ e $Q\left(r_{g}\right)>0$, quando $r \rightarrow r_{g}$ temos que $B\left(r_{g}\right) \rightarrow \infty$ e, conseqüentemente, para todos valores de $C$ em que $A(r) \neq B(r), r_{g}$ é o valor mínimo que coordenada $r$ assume. Uma evidência de que o limite $r \rightarrow r_{g}$ é de fato uma singularidade física é o comportamento do escalar de Kretschmann neste limite. Conforme pode ser visto na expressão (4.26), $\kappa$ diverge com $r \rightarrow r_{g}$. Ou seja, a singularidade é transladada de $r \rightarrow 0$ para $r \rightarrow r_{g}$.

Outro detalhe no gráfico de $B(r)$, figura 5.4, é o fato que para $r<r_{+}$as funções $A(r)$ e $B(r)$ têm sinais opostos. Este problema é uma conseqüência do sistema de coordenadas adotado. Conforme visto na seção 4.2 , as coordenadas $(t, r, \theta, \phi)$ são válidas somente com $r>r_{+}$. Mudando-se para um sistema mais adequado, obtemos explicitamente a métrica com a assinatura +2 .

O comportamento assintótico desta família de soluções foi observado através do gráfico $A(r)-B(r)$ em função de $r / m$. Observa-se que para diferentes valores de $C$, sobretudo para valores que tornam $A(r) \neq B(r)$, a diferença entre estas duas funções tende a zero, ou seja, esta família de soluções tende à solução Schwarzschild anti-de Sitter, $A(r)=B(r)$, quando $r$ cresce, conforme a figura 5.5. Então, as soluções obtidas são assintoticamente anti-de Sitter, tendem à geometria (2.39). Este comportamento assintótico pode, também, ser observado com auxílio do gráfico da coordenada tartaruga em função de $r$. Conforme foi comentado no capítulo anterior, percebemos que esta família de soluções é assintoticamente anti-de Sitter independente do valor da constante $C$, ou seja, a coordenada tartaruga tende a um valor finito quando $r$ cresce, de acordo com a figura 5.6. 


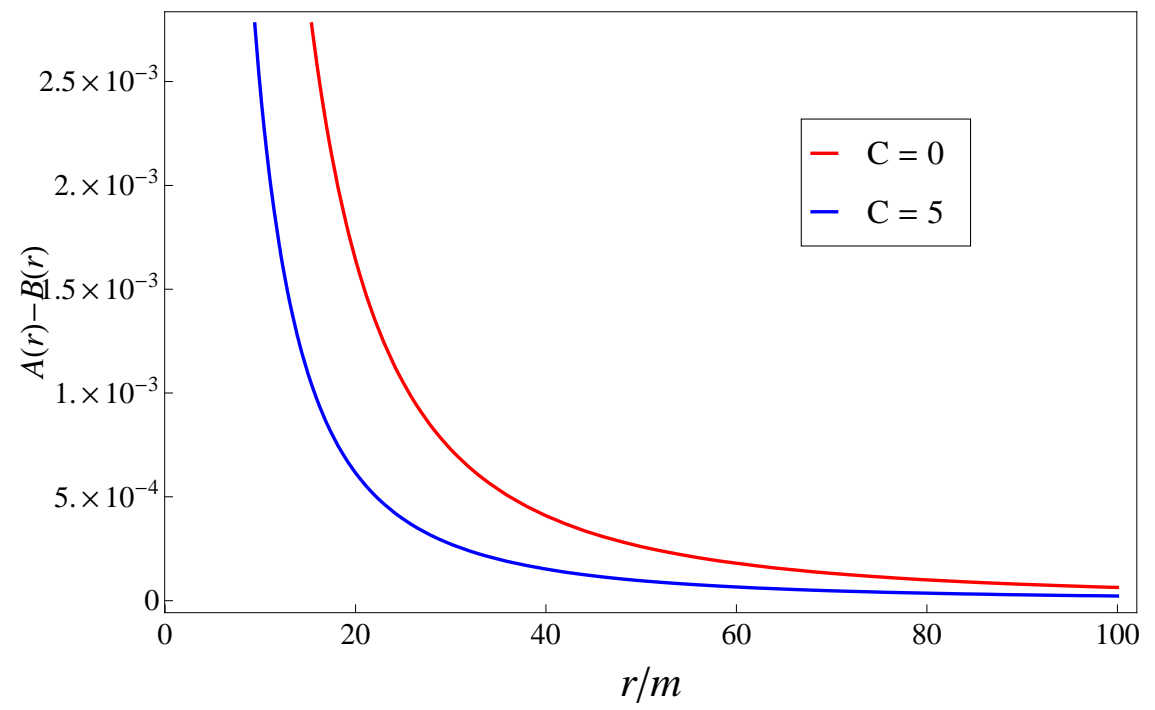

Figura 5.5: Gráfico da difrença $A(r)-B(r)$ em função de $r / m$ para valores de $C$ que tornam $A(r) \neq B(r)$. Neste gráfico foi usado $m=a=1$.

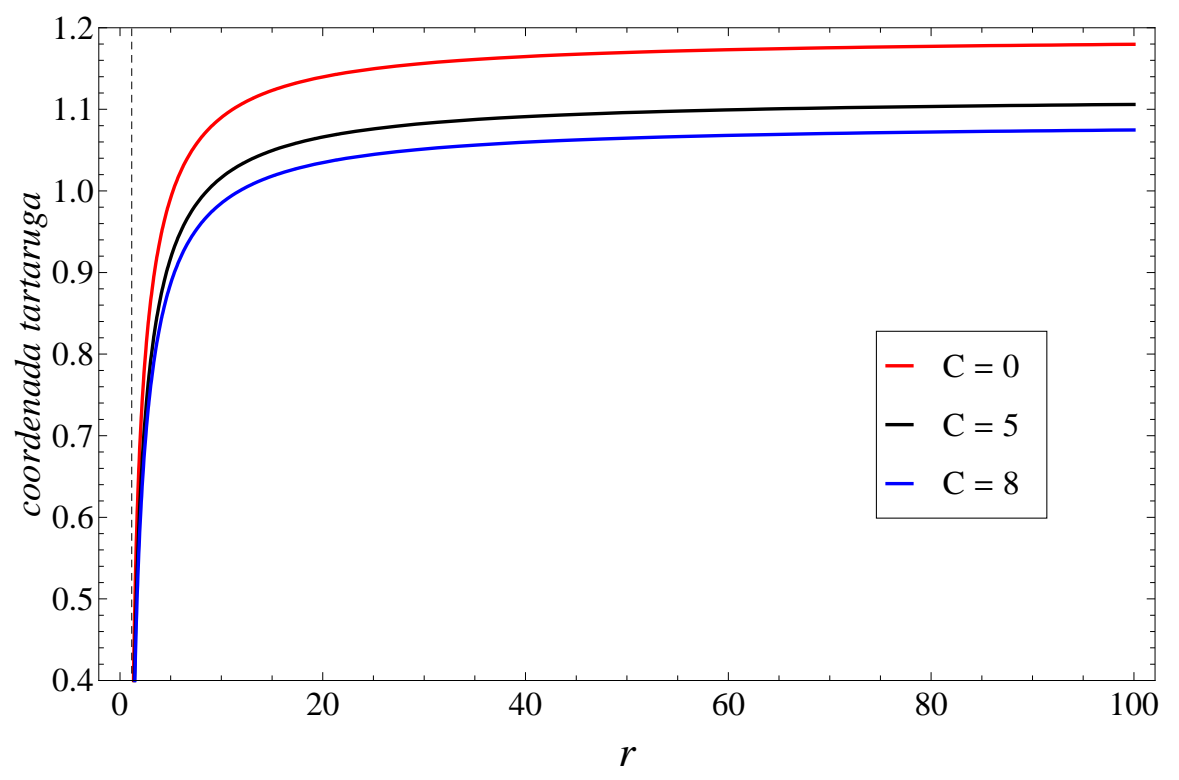

Figura 5.6: Gráfico da coordenada tartaruga, $\tilde{r}^{*}$, em função de $r$. A curva azul representa o caso em que $A(r)=B(r)$. Adotamos para construir este gráfico $m=a=1$ e, conseqüentemente, $r_{+}=1$, indicado pela linha pontilhada.

Podemos obter o tensor energia-momento efetivo na brana para diferentes valores de $C$ com auxílio de (4.39). Com isso, a densidade efetiva, pressões radial e tangencial 
podem ser escritas como

$$
\begin{aligned}
& \rho^{e f}=-\frac{G^{t}{ }_{t}+4 \Lambda_{4 D}}{8 \pi}, \\
& p_{\text {rad }}^{e f}=\frac{G_{r}^{r}+4 \Lambda_{4 D}}{8 \pi}
\end{aligned}
$$

e

$$
p_{\perp}^{e f}=\frac{G^{\theta}{ }_{\theta}+4 \Lambda_{4 D}}{8 \pi} .
$$

O comportamento das componentes do tensor energia-momento efetivo pode ser avaliado através dos gráficos abaixo e nota-se que conforme $r$ cresce as componentes desse tensor tendem a um valor fixo, independente da constante $C$. Tal valor fixo corresponde ao caso onde $A(r)=B(r)$, ou seja, a métrica Schwarzschild anti-de Sitter. Embora estejamos tratando de uma brana sem matéria, temos dois ingredientes a mais: a constante cosmológia e o bulk. Dessa forma, as componentes do tensor energia-momento efetivo não são nulas.

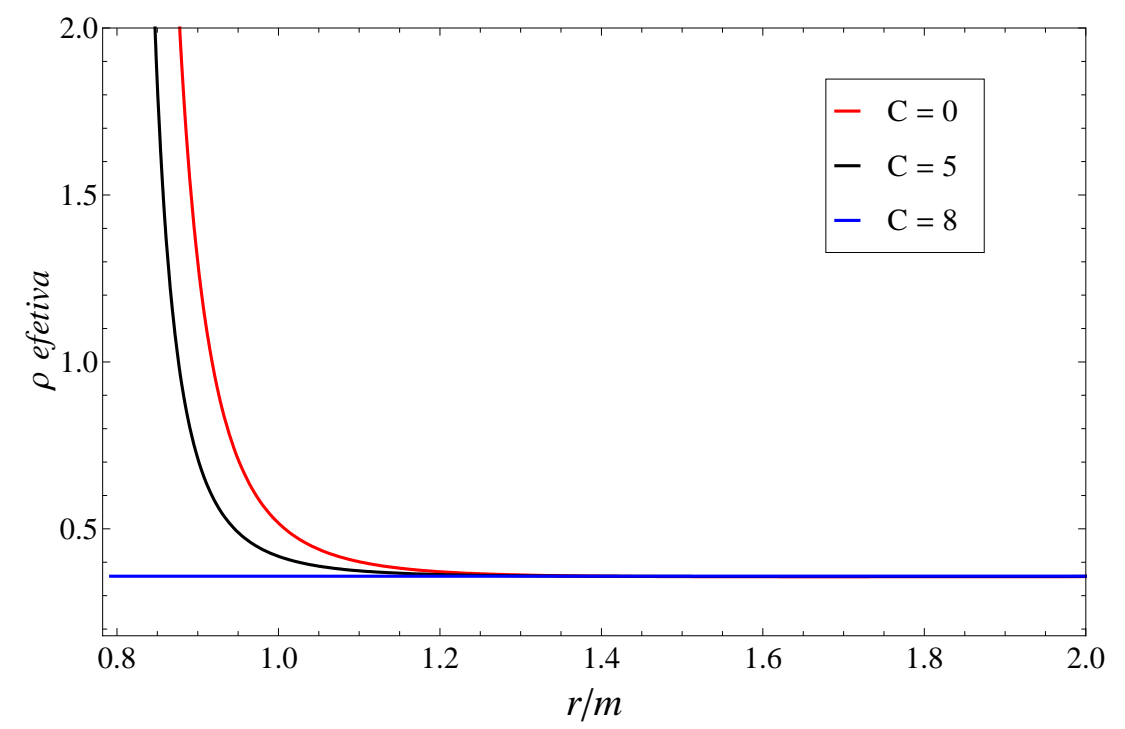

Figura 5.7: Gráfico da densidade efetiva, $\rho^{e f}$, em função de $r / m$. A curva azul é o caso onde $A(r)=B(r)$. Adotamos para construção deste gráfico $m=a=1$ e o raio do horizonte de eventos, conseqüentemente, é $r_{+}=1$. A densidade efetiva diverge, para os casos onde $A(r) \neq B(r)$, quando $r \rightarrow r_{g} \approx 0.8$. 


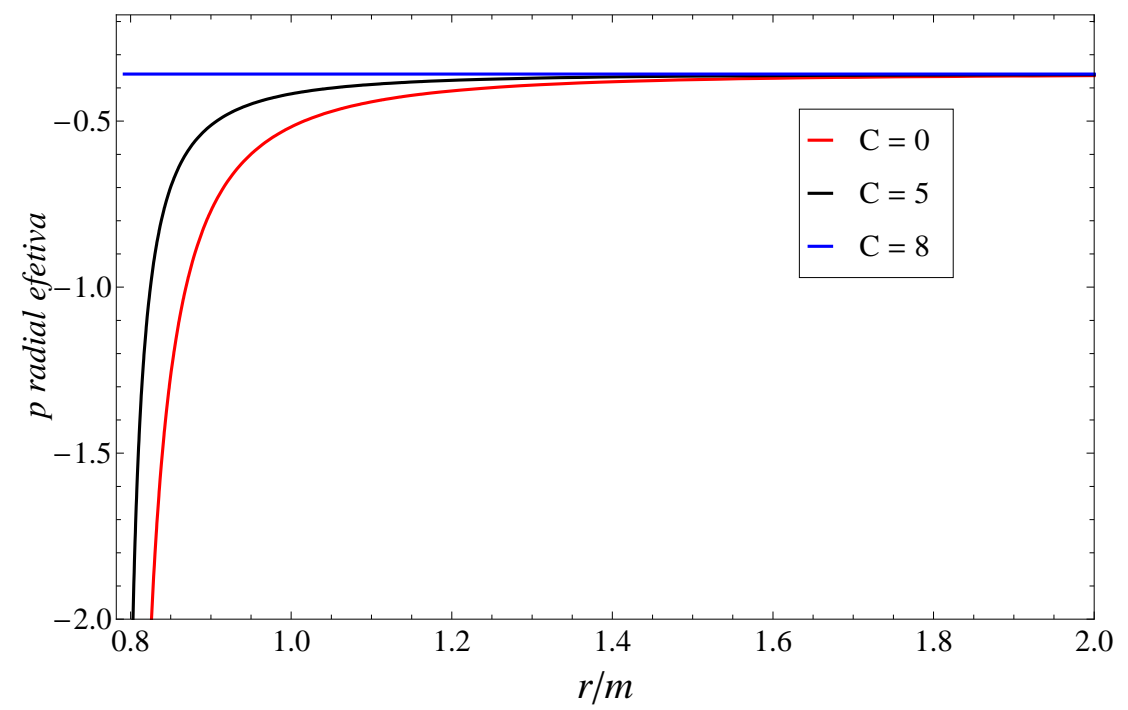

Figura 5.8: Gráfico da pressão radial efetiva, $p_{\text {rad }}^{e f}$, em função de $r / m$. A curva azul é o caso onde $A(r)=B(r)$. Adotamos para construção deste gráfico $m=a=1$ e o raio do horizonte de eventos, conseqüentemente, é $r_{+}=1$. A pressão radial efetiva diverge, para os casos onde $A(r) \neq B(r)$, quando $r \rightarrow r_{g} \approx 0.8$.

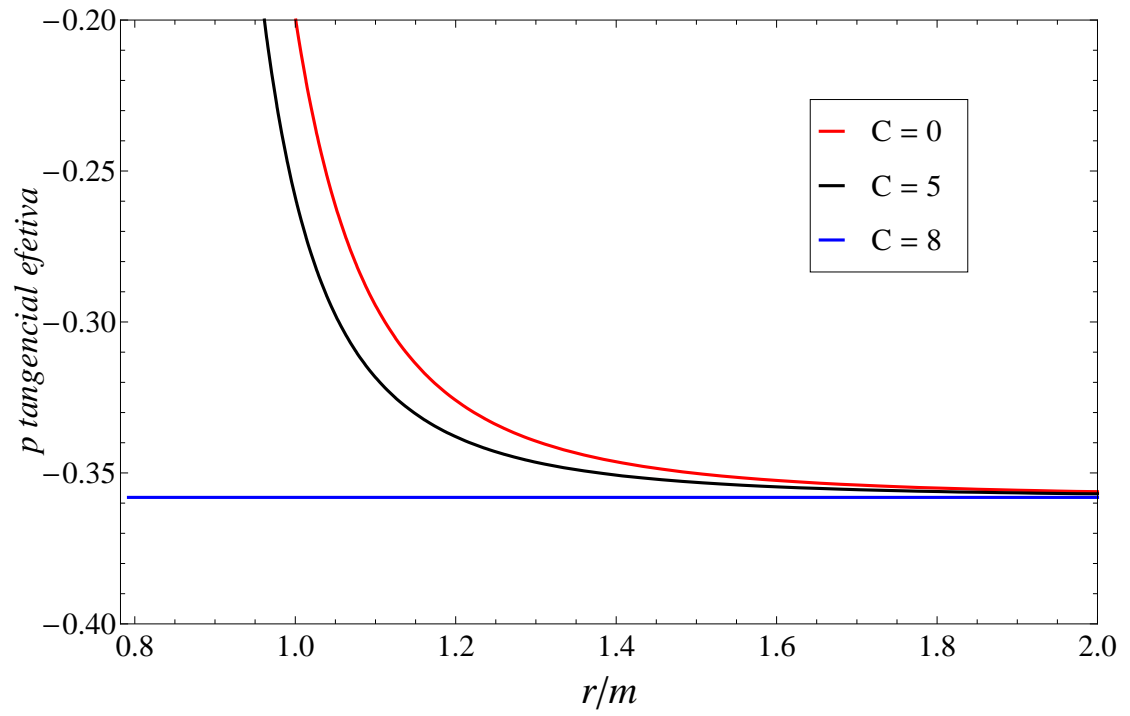

Figura 5.9: Gráfico da pressão tangencial efetiva, $p_{\perp}^{e f}$, em função de $r / m$. A curva azul é o caso onde $A(r)=B(r)$. Adotamos para construção deste gráfico $m=a=1$ e o raio do horizonte de eventos, conseqüentemente, é $r_{+}=1$. A pressão tangencial efetiva diverge, para os casos onde $A(r) \neq B(r)$, quando $r \rightarrow r_{g} \approx 0.8$. 


\subsubsection{Comportamento próximo ao horizonte}

Agora estamos interessados no comportamento desta família de soluções próximo ao horizonte de eventos, $r_{+}$. Conseguiremos isto escrevendo (5.20) como

$$
A(r)=A_{r}\left(r_{+}\right)\left(r-r_{+}\right)+\mathcal{O}\left[(r-r)^{2}\right] .
$$

Além disso, $A_{r}$ e $g(r)$ podem ser escritas como

$$
A_{r}=A_{r}\left(r_{+}\right)+A_{r r}\left(r_{+}\right)\left(r-r_{+}\right)+\mathcal{O}\left[\left(r-r_{+}\right)\right]
$$

e

$$
\begin{aligned}
g(r) & =4 A(r)+r A_{r}(r) \\
& =A_{r}\left(r_{+}\right) r_{+}+\mathcal{O}\left[\left(r-r_{+}\right)\right] .
\end{aligned}
$$

A função $\Gamma$, dada por (4.31), foi calculada próxima ao horizonte, e as exponenciais, usadas no cálculo de $B(r)$, foram escritas como

$$
e^{3 \Gamma}=1+\mathcal{O}\left[\left(r-r_{+}\right)\right]
$$

e

$$
e^{-3 \Gamma}=1+\mathcal{O}\left[\left(r-r_{+}\right)\right]
$$

A função $Q(r)$ fica

$$
Q(r)=2-r^{2} R=Q\left(r_{+}\right)+\mathcal{O}\left[\left(r-r_{+}\right)\right],
$$

e $1 / g(r)^{2}$ pode ser escrita como

$$
\frac{1}{g(r)^{2}}=\frac{1}{A_{r}\left(r_{+}\right)^{2} r_{+}^{2}}+\mathcal{O}\left[\left(r-r_{+}\right)\right]
$$

Conseqüentemente, $B(r)$, dada por (4.27) e (4.29), pôde ser escrita como

$$
\begin{aligned}
B(r) & =\frac{2 A e^{3 \Gamma}}{r g(r)^{2}}\left[\int_{r_{+}}^{r} g(r) Q(r) e^{-3 \Gamma} d r+C\right] \\
& =\frac{2 C}{A_{r}\left(r_{+}\right) r_{+}^{2}}\left(r-r_{+}\right)+\frac{2 Q\left(r_{+}\right)}{r_{+}^{2}}\left(r-r_{+}\right)^{2}+\mathcal{O}\left[\left(r-r_{+}\right)^{3}\right],
\end{aligned}
$$

próxima do horizonte. Dessa forma, uma família de funções $B(r)$ foi calculada ana- 
liticamente em torno de $r_{+}$, e para a constante de integração, $C$, diferente de zero temos

$$
B(r)=\frac{2 C}{A_{r}\left(r_{+}\right) r_{+}^{3}}\left(r-r_{+}\right)+\mathcal{O}\left[\left(r-r_{+}\right)^{2}\right],
$$

onde para $C=C_{0}$, com

$$
C_{0}=\frac{1}{2} A_{r}\left(r_{+}\right)^{2} r_{+}^{3},
$$

temos $A(r)=B(r)$, ou seja, a métrica Schwarzschild anti-de Sitter. O resultado em (5.51) foi testado extensivamente com as ferramentas numéricas desenvolvidas. Nos gráficos apresentados nesta dissertação, fazendo $m=a=1 \mathrm{e}$, por conseqüência, $r_{+}=1$, o valor de $C$ que torna $A(r)=B(r)$ é $C=C_{0}=8$. Para o caso em que $C=0$, a função $B(r)$ próxima ao horizonte será

$$
B(r)=\frac{2 Q\left(r_{+}\right)}{r_{+}^{2}}\left(r-r_{+}\right)^{2}+\mathcal{O}\left[\left(r-r_{+}\right)^{3}\right] .
$$

Podemos escrever a função $h(r)$, dada por (4.14), quando $C \neq 0$, com auxílio de auxílio (5.42) e (5.50), como

$$
h(r)=\sqrt{\frac{2 C}{r_{+}^{3}}}\left(r-r_{+}\right)+\mathcal{O}\left[\left(r-r_{+}\right)^{2}\right],
$$

e a gravidade superficial, dada por (4.17), neste caso fica

$$
\kappa_{+}=\sqrt{\frac{C}{2 r_{+}^{3}}} .
$$

Para o caso em que $C=0$, conhecido como caso extremo, usamos (5.42) e (5.52) para obter

$$
h(r)=\frac{\sqrt{2 Q\left(r_{+}\right) A_{r}\left(r_{+}\right)}}{r_{+}}\left(r-r_{+}\right)^{\frac{3}{2}}+\mathcal{O}\left[\left(r-r_{+}\right)^{\frac{5}{2}}\right],
$$

sendo a gravidade superficial nula neste caso

$$
\kappa_{+}=0 .
$$

Em geral, com $C \neq 0$, a gravidade superficial é uma função de $m, a$ e $C$, dada por

$$
\kappa_{+}=\left[\frac{3 \sqrt{3} \mathcal{G}(m, a) C}{2\left(\mathcal{G}(m, a)^{2 / 3}-a^{2}\right)^{3}}\right]^{1 / 2},
$$


com $\mathcal{G}(m, a)$ apresentada em (5.25).

Como foi dito no capítulo anterior, existe uma analogia entre a gravidade superficial e a temperatura na termodinâmica. A lei zero da termodinâmica estabelece que a temperatura de um corpo em equilíbrio é constante, da mesma forma que $\kappa_{+}$é constante no horizonte de eventos de um buraco negro estacionário. Buracos negros extremos são interessantes do ponto de vista da terceira lei generalizada da termodinâmica. Esta lei afirma que buracos negros com $\kappa_{+}=0$ não poderiam ser gerados por processos dinâmicos a partir de buracos negros com $\kappa_{+} \neq 0$ [28]. O gráfico da gravidade superficial, para determinados valores de $C$, em função de $m$ e $a$ é exibido na figura 5.10.

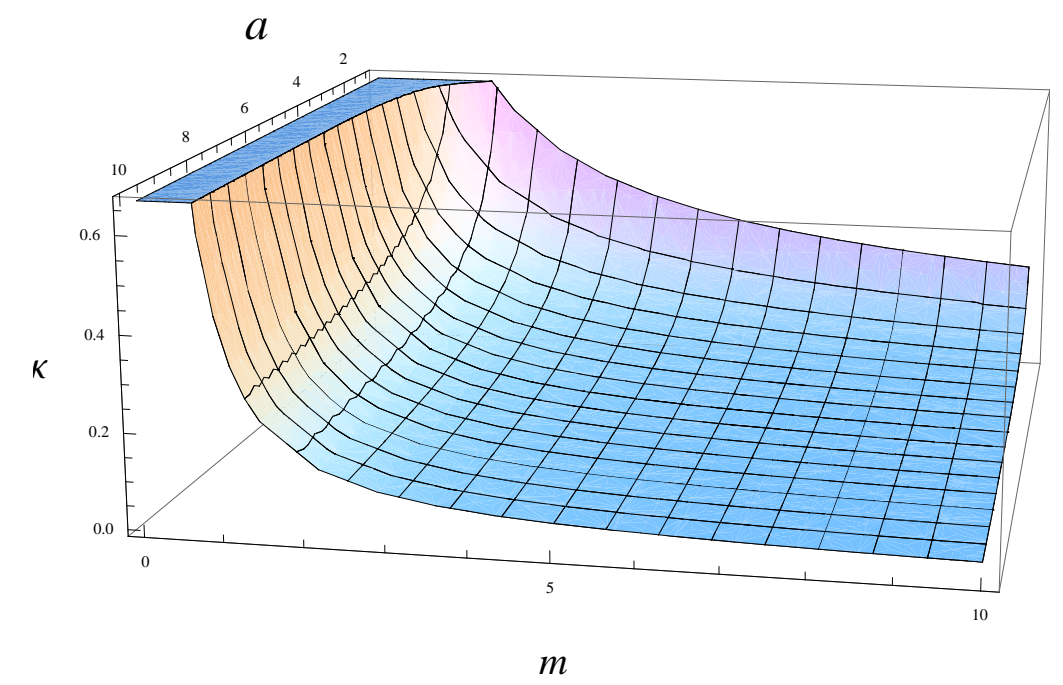

Figura 5.10: Gráfico da gravidade superficial em função da massa $m$ e $a$. Utilizamos $C=2$.

Através de (4.4), podemos obter a coordenada quase global $u(r)$ e calcular a função $\mathcal{A}(u)$, próxima a $r_{+}$, para determinar a ordem do horizonte de eventos. Para o caso em que $C>0$, temos um $r(u)$ na forma

$$
r \approx r_{+}+\frac{1}{A_{r}\left(r_{+}\right)} \sqrt{\frac{2 C}{r_{+}^{3}}}(u-h),
$$

que nos conduz a

$$
\mathcal{A}(u) \approx \sqrt{\frac{2 C}{r_{+}^{3}}}(u-h),
$$


com um horizonte simples e com a constante de proporcionalidade igual a $2 \kappa_{+}$. Para o caso $C=0$, temos

$$
r \approx r_{+}+\frac{Q\left(r_{+}\right)^{2}}{2 A_{r}\left(r_{+}\right)^{2} r_{+}^{4}}(u-h)^{2},
$$

com $\mathcal{A}(u)$ indicando que o horizonte de eventos, $u=h$, neste caso tem ordem 2

$$
\mathcal{A}(u) \approx \frac{Q\left(r_{+}\right)^{2}}{2 A_{r}\left(r_{+}\right) r_{+}^{4}}(u-h)^{2} .
$$




\section{Capítulo 6}

\section{Perturbações Escalares}

Neste capítulo estudamos a estabilidade à evolução de um campo escalar da família de soluções obtida no capítulo anterior. Os pioneiros no estudo sobre estabilidade de buracos negros foram Regge e Wheeler quando em 1957 no artigo [30] estudaram a solução de Schwarzschild e mostraram sua estabilidade. É interessante considerar a dinâmica de campos para o caso de espaços-tempos assintoticamente anti-de Sitter. Além de aspectos ligados à evolução do campo teste, a descoberta da correspondência anti-de Sitter/teoria de campo conforme (AdS/CFT) torna a investigação mais atraente.

\subsection{Introduzindo o campo escalar}

Em geometrias esfericamente simétricas

$$
d s^{2}=A(r) d t^{2}-\frac{d r^{2}}{B(r)}-r^{2}\left(d \theta+\operatorname{sen}^{2} \theta d \phi^{2}\right),
$$

a propagação de campos escalares pode ser tratada com bastante generalidade, se considerarmos o background como fixo, e, portanto, o tensor energia-momento associado ao campo escalar desprezível. A equação de Klein-Gordon para campos escalares não massivos, $\Phi$, é dada por

$$
\square \Phi=0,
$$

onde $\square$ é o operador d'Alembertiano

$$
\square=g^{\mu \nu} \nabla_{\mu} \nabla_{\nu},
$$


A equação (6.3) pode ser escrita em termos das coordenadas esféricas $(t, r, \theta, \phi)$

$$
\square=\frac{1}{\sqrt{-g}} \partial_{\mu}\left(\sqrt{-g} g^{\mu \nu} \partial_{\nu}\right)
$$

onde $g=\operatorname{det}\left(g_{\mu \nu}\right)$. Como a matriz $\left(g_{\mu \nu}\right)$ é diagonal, o seu determinante é simplesmente o produto dos seus elementos da diagonal principal, e, portanto,

$$
\sqrt{-g}=\sqrt{\frac{A(r)}{B(r)}} r^{2} \operatorname{sen} \theta
$$

Podemos escrever (6.4) em duas partes

$$
\square=\square_{2}+\nabla_{2}
$$

onde os operadores $\square_{2}$ e $\nabla_{2}$ são escritos como

$$
\square_{2}=\frac{1}{\sqrt{-g}}\left[\partial_{0}\left(\sqrt{-g} g^{00} \partial_{0}\right)+\partial_{1}\left(\sqrt{-g} g^{11} \partial_{1}\right)\right]
$$

e

$$
\nabla_{2}=\frac{1}{\sqrt{-g}}\left[\partial_{2}\left(\sqrt{-g} g^{22} \partial_{2}\right)+\partial_{3}\left(\sqrt{-g} g^{33} \partial_{3}\right)\right] .
$$

Substituindo os elementos da métrica (6.1) em $\square_{2}$ e $\nabla_{2}$, obtemos, após os devidos cancelamentos,

$$
\square_{2}=\frac{1}{A(r)} \frac{\partial^{2}}{\partial t^{2}}-B(r) \frac{\partial^{2}}{\partial r^{2}}-\left[\frac{2}{r}+\frac{A_{r}(r)}{2 A(r)}+\frac{B_{r}(r)}{2 B(r)}\right] B(r) \frac{\partial}{\partial r},
$$

onde subscrito $r$ indica $d / d r$, e

$$
\nabla_{2}=-\frac{1}{r^{2}}\left[\frac{1}{\operatorname{sen} \theta} \frac{\partial}{\partial \theta}\left(\operatorname{sen} \theta \frac{\partial}{\partial \theta}\right)+\frac{1}{\operatorname{sen}^{2} \theta} \frac{\partial^{2}}{\partial \phi^{2}}\right]
$$

Vemos, então, que o operador $\nabla_{2}$ é o operador Laplaciano em 2 dimensões escrito em coordenadas esféricas.

Como o operador $\square_{2}$ só atua nas variáveis $t$ e $r$, enquanto que o operador $\nabla_{2}$ só atua nas variáveis angulares, podemos expandir o campo $\Phi$ em multipolos, ou seja,

$$
\Phi=\sum_{\ell, m} \psi_{\ell}(t, r) \mathrm{Y}_{\ell m}(\theta, \phi)
$$


onde $\mathrm{Y}_{\ell m}$ são os harmônicos esféricos, auto-funções do operador Laplaciano bidimensional

$$
\nabla_{2} \mathrm{Y}_{\ell m}=\frac{1}{r^{2}} \ell(\ell+1) \mathrm{Y}_{\ell m}
$$

soluções de

$$
\frac{1}{\operatorname{sen} \theta} \frac{\partial}{\partial \theta}\left(\operatorname{sen} \theta \frac{\partial \mathrm{Y}_{\ell m}}{\partial \theta}\right)+\frac{1}{\operatorname{sen}^{2} \theta} \frac{\partial^{2} \mathrm{Y}_{\ell m}}{\partial \phi^{2}}+\ell(\ell+1) \mathrm{Y}_{\ell m}=0
$$

Substituindo o Ansatz (6.11) e (6.12) na equação de movimento (6.2), obtemos

$$
\begin{gathered}
\sum_{\ell, m}\left\{\square_{2}\left(\psi_{\ell}\right) \mathrm{Y}_{\ell m}+\psi_{\ell} \nabla_{2} \mathrm{Y}_{\ell m}\right\}=0 \\
\sum_{\ell, m} \mathrm{Y}_{\ell m}\left\{\square_{2}\left(\psi_{\ell}\right)+\frac{\ell(\ell+1)}{r^{2}} \psi_{\ell}\right\}=0 .
\end{gathered}
$$

Como as funções $\mathrm{Y}_{\ell m}$ formam um conjunto completo, cada termo da somatória anterior deve se anular. Assim, ficamos com equações independentes para cada modo $\ell$

$$
\square_{2}\left(\psi_{\ell}\right)+\frac{\ell(\ell+1)}{r^{2}} \psi_{\ell}=0
$$

Com auxílio de (6.9), a equação acima fica

$$
\frac{\partial^{2} \psi_{\ell}}{\partial t^{2}}-A B\left(\frac{\partial^{2} \psi_{\ell}}{\partial r^{2}}\right)-\left[\frac{2}{r}+\frac{A_{r}}{2 A}+\frac{B_{r}}{2 B}\right] A B \frac{\partial \psi_{\ell}}{\partial r}=-A\left[\frac{\ell(\ell+1)}{r^{2}}\right] \psi_{\ell}
$$

Temos então para cada modo $\ell$ uma equação parcial linear hiperbólica para a função $\psi_{\ell}(t, r)$, que descreve a dinâmica da componente $\ell$ do campo escalar $\Phi$.

Para resolvermos a equação (6.16) numericamente, substituimos a coordenada $r$ pela coordenada tartaruga $\tilde{r}^{*}$ e definimos

$$
\psi(r, t)=\varphi(r, t) \rho(r)
$$

onde o subscrito $\ell$ será abandonado a partir de agora,

$$
\alpha(r)=A(r) B(r)
$$

e

$$
r=r\left(\tilde{r}^{*}\right)
$$


Depois de substituir as relações acima em (6.16), obtemos

$$
\begin{aligned}
& \rho \frac{\partial^{2} \varphi}{\partial t^{2}}-\alpha \frac{\partial \tilde{r}^{*}}{\partial r} \frac{\partial}{\partial r}(\varphi \rho)\left[\frac{2}{r}+\right.\left.\frac{1}{2 A} \frac{\partial \tilde{r}^{*}}{\partial r} \frac{\partial A}{\partial \tilde{r}^{*}}+\frac{1}{2 B} \frac{\partial \tilde{r}^{*}}{\partial r} \frac{\partial B}{\partial \tilde{r}^{*}}\right]- \\
&-\alpha \frac{\partial \tilde{r}^{*}}{\partial r} \frac{\partial}{\partial \tilde{r}^{*}}\left[\frac{\partial \tilde{r}^{*}}{\partial r} \frac{\partial}{\partial \tilde{r}^{*}}(\varphi \rho)\right]=-A\left[\frac{\ell(\ell+1)}{r^{2}}\right] \varphi \rho .
\end{aligned}
$$

Parte do segundo termo do lado esquerdo da equação acima pode ser escrito como

$$
\begin{aligned}
\frac{2}{r}+\frac{1}{2 A} \frac{\partial \tilde{r}^{*}}{\partial r} \frac{\partial A}{\partial \tilde{r}^{*}}+\frac{1}{2 B} \frac{\partial \tilde{r}^{*}}{\partial r} \frac{\partial B}{\partial \tilde{r}^{*}} & =\frac{2}{r}+\frac{1}{2} \frac{\partial \tilde{r}^{*}}{\partial r}\left(\frac{1}{A} \frac{\partial A}{\partial \tilde{r}^{*}}+\frac{1}{B} \frac{\partial B}{\partial \tilde{r}^{*}}\right) \\
& =\frac{2}{r}+\frac{1}{2} \frac{\partial \tilde{r}^{*}}{\partial r}\left(\frac{\partial}{\partial r}(\ln A+\ln B)\right) \\
& =\frac{2}{r}+\frac{1}{2 \alpha} \frac{\partial \tilde{r}^{*}}{\partial r} \frac{\partial \alpha}{\partial \tilde{r}^{*}}
\end{aligned}
$$

Substituindo (6.21) na equação (6.20) dividida por $\rho$, temos

$$
\begin{aligned}
\frac{\partial^{2} \varphi}{\partial t^{2}}-\frac{\alpha}{\rho} \frac{\partial \tilde{r}^{*}}{\partial r}\left(\varphi \frac{\partial \rho}{\partial \tilde{r}^{*}}+\rho \frac{\partial \varphi}{\partial \tilde{r}^{*}}\right)\left[\frac{2}{r}+\frac{1}{2 \alpha} \frac{\partial \tilde{r}^{*}}{\partial r} \frac{\partial \alpha}{\partial \tilde{r}^{*}}\right] & - \\
-\frac{\alpha}{\rho} \frac{\partial \tilde{r}^{*}}{\partial r}\left(\varphi \frac{\partial \rho}{\partial \tilde{r}^{*}}+\rho \frac{\partial \varphi}{\partial \tilde{r}^{*}}\right) \frac{\partial}{\partial \tilde{r}^{*}}\left(\frac{\partial \tilde{r}^{*}}{\partial r}\right) & - \\
-\frac{\alpha}{\rho}\left(\frac{\partial \tilde{r}^{*}}{\partial r}\right)^{2}\left(\varphi \frac{\partial^{2} \rho}{\partial \tilde{r}^{* 2}}+2 \frac{\partial \varphi}{\partial \tilde{r}^{*}} \frac{\partial \rho}{\partial \tilde{r}^{*}}+\rho \frac{\partial^{2} \varphi}{\partial \tilde{r}^{* 2}}\right) & =-A\left[\frac{\ell(\ell+1)}{r^{2}}\right] \varphi .
\end{aligned}
$$

Podemos escrever a equação acima reagrupando termos da seguinte forma

$$
\begin{gathered}
\frac{\partial^{2} \varphi}{\partial t^{2}}-\alpha\left(\frac{\partial \tilde{r}^{*}}{\partial r}\right)^{2} \frac{\partial^{2} \varphi}{\partial \tilde{r}^{* 2}}-\left[\alpha \frac{\partial \tilde{r}^{*}}{\partial r}\left(\frac{2}{r}+\frac{1}{2 \alpha} \frac{\partial \tilde{r}^{*}}{\partial r} \frac{\partial \alpha}{\partial \tilde{r}^{*}}\right)+\alpha \frac{\partial \tilde{r}^{*}}{\partial r} \frac{\partial}{\partial \tilde{r}^{*}}\left(\frac{\partial \tilde{r}^{*}}{\partial r}\right)+\right. \\
\left.+\frac{2 \alpha}{\rho}\left(\frac{\partial \tilde{r}^{*}}{\partial r}\right)^{2} \frac{\partial \rho}{\partial \tilde{r}^{*}}\right] \frac{\partial \varphi}{\partial \tilde{r}^{*}}-\left[\frac{\alpha}{\rho} \frac{\partial \tilde{r}^{*}}{\partial r} \frac{\partial \rho}{\partial \tilde{r}^{*}}\left(\frac{2}{r}+\frac{1}{2 \alpha} \frac{\partial \tilde{r}^{*}}{\partial r} \frac{\partial \alpha}{\partial \tilde{r}^{*}}\right)+\frac{\alpha}{\rho} \frac{\partial \tilde{r}^{*}}{\partial r} \frac{\partial}{\partial \tilde{r}^{*}}\left(\frac{\partial \tilde{r}^{*}}{\partial r}\right) \frac{\partial \rho}{\partial \tilde{r}^{*}}+\right. \\
\left.+\frac{\alpha}{\rho}\left(\frac{\partial \tilde{r}^{*}}{\partial r}\right)^{2} \frac{\partial^{2} \rho}{\partial \tilde{r}^{*}}\right] \varphi=-A \varphi\left[\frac{\ell(\ell+1)}{r^{2}}\right] .
\end{gathered}
$$

Para que a equação acima fique do tipo

$$
-\frac{\partial^{2} \varphi(r, t)}{\partial t^{2}}+\frac{\partial^{2} \varphi(r, t)}{\partial \tilde{r}^{* 2}}=V_{e f}(r) \varphi(r, t),
$$

devemos impor

$$
\alpha\left(\frac{\partial \tilde{r}^{*}}{\partial r}\right)^{2}=1
$$




$$
\begin{gathered}
\alpha \frac{\partial \tilde{r}^{*}}{\partial r}\left(\frac{2}{r}+\frac{1}{2 \alpha} \frac{\partial \tilde{r}^{*}}{\partial r} \frac{\partial \alpha}{\partial \tilde{r}^{*}}\right)+\alpha \frac{\partial \tilde{r}^{*}}{\partial r} \frac{\partial}{\partial \tilde{r}^{*}}\left(\frac{\partial \tilde{r}^{*}}{\partial r}\right)+\frac{2 \alpha}{\rho}\left(\frac{\partial \tilde{r}^{*}}{\partial r}\right)^{2} \frac{\partial \rho}{\partial \tilde{r}^{*}}=0 \\
V_{e f}(r)=A\left[\frac{\ell(\ell+1)}{r^{2}}\right]-V(r)
\end{gathered}
$$

onde

$$
V(r)=\frac{\alpha}{\rho} \frac{\partial \tilde{r}^{*}}{\partial r} \frac{\partial \rho}{\partial \tilde{r}^{*}}\left(\frac{2}{r}+\frac{1}{2 \alpha} \frac{\partial \tilde{r}^{*}}{\partial r} \frac{\partial \alpha}{\partial \tilde{r}^{*}}\right)+\frac{\alpha}{\rho} \frac{\partial \tilde{r}^{*}}{\partial r} \frac{\partial}{\partial \tilde{r}^{*}}\left(\frac{\partial \tilde{r}^{*}}{\partial r}\right) \frac{\partial \rho}{\partial \tilde{r}^{*}}+\frac{\alpha}{\rho}\left(\frac{\partial \tilde{r}^{*}}{\partial r}\right)^{2} \frac{\partial^{2} \rho}{\partial \tilde{r}^{*}}
$$

Portanto, a equação (6.25) fica

$$
\frac{d \tilde{r}^{*}}{d r}=\frac{1}{\sqrt{\alpha}}=\frac{1}{h(r)}
$$

que recupera a definição apresentada em (4.13). A partir de agora usaremos a derivada $d$ e não mais a derivada parcial $\partial$. Logo em seguida, substituindo (6.29) em (6.26), obtemos

$$
\begin{gathered}
\frac{2}{r}+\frac{1}{2 \alpha} \frac{d \alpha}{d r}-\frac{1}{2 \alpha} \frac{d \alpha}{d r}+\frac{2}{\rho} \frac{d \rho}{d r}=0, \\
\frac{d \rho}{d r}=-\frac{\rho}{r} \Rightarrow \rho=\frac{1}{r} .
\end{gathered}
$$

Assim, a função de onda (6.11), que descreve o campo escalar não massivo, fica

$$
\Phi(r, t, \theta, \phi)=\sum_{\ell, m} \frac{\varphi_{\ell}(r, t)}{r} Y_{\ell m}(\theta, \phi)
$$

Substituindo a expressão para $\rho$ na equação (6.28) e com auxílio de (6.29), chegamos à forma final do potencial

$$
V(r)=-\frac{1}{2 r} \frac{d \alpha}{d r}
$$

e do potencial efetivo

$$
V_{e f}(r)=A\left[\frac{\ell(\ell+1)}{r^{2}}\right]+\frac{1}{2 r} \frac{d \alpha}{d r}
$$

que pode ser escrito como

$$
V_{e f}(r)=A\left[\frac{\ell(\ell+1)}{r^{2}}\right]+\frac{1}{2 r}\left[A(r) B_{r}(r)+B(r) A_{r}(r)\right]
$$

Pelo que foi visto no capítulo anterior, $V_{e f}$ é positivo em $r_{+}<r<\infty$, pois $A(r)$ e $B(r)$ são funções positivas monotonicamente crescentes neste intervalo (veja figura 5.4). Este é um resultado importante, pois a positividade do potencial efetivo garante 
a estabilidade do campo escalar, conforme pode ser visto em [31].

Vamos estudar o comportamento de $V_{e f}\left(\tilde{r}^{*}\right)$ próximo ao horizonte para a família de soluções assintoticamente anti-de Sitter obtida no capítulo anterior. Para o caso em que $C \neq 0$, a função $h(r)$ em primeira ordem de aproximação, de acordo com (5.53), é dada por

$$
h(r)=2 \kappa_{+}\left(r-r_{+}\right)+\mathcal{O}\left[\left(r-r_{+}\right)^{\frac{3}{2}}\right]
$$

e a coordenada tartaruga, $\tilde{r}^{*}(r)$, definida em (4.13), torna-se

$$
\tilde{r}^{*}(r)=\frac{1}{2 \kappa_{+}} \ln \left(r-r_{+}\right)+\mathcal{O}\left[\left(r-r_{+}\right)^{\frac{1}{2}}\right],
$$

ou seja,

$$
r\left(\tilde{r}^{*}\right)=r_{+}+e^{2 \kappa+\tilde{r}^{*}}\left(1+\mathcal{O}\left[\left(r-r_{+}\right)^{\frac{1}{2}}\right]\right) .
$$

Então, o potencial dado por (6.34), com auxílio de (5.42) e (5.50), quando $\ell \geq 0$, será

$$
V_{e f}(r) \sim\left(r-r_{+}\right)+\mathcal{O}\left[\left(r-r_{+}\right)^{2}\right]
$$

e com auxílio de (6.37) fica

$$
V_{e f}\left(\tilde{r}^{*}\right)=V_{e f}\left(r\left(\tilde{r}^{*}\right)\right) \sim e^{2 \kappa_{+} \tilde{r}^{*}} .
$$

Quando nos aproximamos do horizonte, $\tilde{r}^{*} \rightarrow-\infty$ e o potencial decai exponencialmente.

Para o caso em que $C=0$, a função $h(r)$, de acordo com (5.55), pode ser escrita como

$$
h(r)=\frac{\sqrt{2 Q\left(r_{+}\right) A_{r}\left(r_{+}\right)}}{r_{+}}\left(r-r_{+}\right)^{\frac{3}{2}}+\mathcal{O}\left[\left(r-r_{+}\right)^{2}\right],
$$

e a coordenada tartaruga $\tilde{r}^{*}(r)$ torna-se

$$
\tilde{r}^{*}(r)=-\frac{2 r_{+}}{\sqrt{2 Q\left(r_{+}\right) A_{r}\left(r_{+}\right)}}\left(r-r_{+}\right)^{-\frac{1}{2}} .
$$

Como $\tilde{r}^{*}<0$, próximo ao horizonte, podemos escrever (6.41) na seguinte forma

$$
\left(r-r_{+}\right)^{\frac{1}{2}}=-\frac{2 r_{+}}{\sqrt{2 Q\left(r_{+}\right) A_{r}\left(r_{+}\right)}} \frac{1}{\tilde{r}^{*}},
$$

ou seja,

$$
r\left(\tilde{r}^{*}\right)=r_{+}+\frac{2 r_{+}^{2}}{Q\left(r_{+}\right) A_{r}\left(r_{+}\right)} \frac{1}{\tilde{r}^{* 2}} .
$$


Assim como no caso $C \neq 0$, utilizando (5.42) e (5.52), o potencial pode ser escrito como (6.38) quando $\ell>0$, e, com auxílio de (6.43), pode ser expresso como função da coordenada tartaruga

$$
V_{e f}\left(\tilde{r}^{*}\right) \sim \frac{1}{\tilde{r}^{* 2}} .
$$

Quando $\ell=0$, o potencial efetivo será

$$
V_{e f}(r) \sim\left(r-r_{+}\right)^{2}+\mathcal{O}\left[\left(r-r_{+}\right)^{3}\right],
$$

e pode, igualmente, ser escrito como função de $\tilde{r}^{*}$

$$
V_{e f}\left(\tilde{r}^{*}\right) \sim \frac{1}{\tilde{r}^{* 4}} .
$$

Então, quando $C=0$, o potencial efetivo tende a zero como uma lei de potência próximo ao horizonte de eventos.

Podemos escrever (6.31) de tal forma que a parte temporal seja expressa através de uma exponencial, ou seja,

$$
\Phi(r, t, \theta, \phi)=\sum_{\ell, m} \frac{\varphi_{\ell}(r)}{r} Y_{\ell m}(\theta, \phi) e^{-i \omega t},
$$

e, conseqüentemente, (6.24) toma a forma de uma equação do tipo Schröedinger

$$
\frac{\partial^{2} \varphi(r)}{d \tilde{r}^{* 2}}+\left(\omega^{2}-V_{e f}(r)\right) \varphi(r)=0,
$$

que é a equação que governa a evolução do campo escalar não massivo para um dado modo $\ell$.

O problema que estamos tratando, a evolução do campo escalar não massivo, é parecido com o problema de ondas eletromagnéticas emitidas por uma fonte numa cavidade, sendo este fenômeno governado por uma equação do tipo (6.24). A geometria da cavidade impõe uma condição de contorno ao problema fazendo com que ondas eletromagnéticas existam somente dentro. Para uma geometria assintoticamente plana, como Schwarzschild, o potencial cai como uma lei de potência com $r \rightarrow \infty$ e existem soluções de (6.48) para cada $\omega$ que correspondem a uma onda originária do infinito, sendo parte absorvida e parte refletida pelo buraco negro. As freqüências de oscilação, $\omega$, são chamadas freqüências quase normais e são determinadas pelo potencial $V_{e f}$, que é determinado pela geometria do espaço-tempo. Conseqüentemente, as freqüências quase 
normais podem indicar a estrutura do espaço-tempo, inclusive a existência de dimensões extras como propõe Maartens et al. em [29], da mesma forma que as freqüências das ondas eletromagnéticas no problema da cavidade são determinadas por parâmetros da própria cavidade. Numa geometria assintoticamente plana, as condições de contorno impostas são

$$
\psi \sim e^{i \omega \tilde{r}^{*}} \quad \text { quando } \quad \tilde{r}^{*} \rightarrow-\infty
$$

e

$$
\psi \sim e^{-i \omega \tilde{r}^{*}} \text { quando } \quad \tilde{r}^{*} \rightarrow \infty .
$$

Para geometrias assintoticamente anti-de Sitter, como a família de soluções obtida no capítulo anterior, o potencial efetivo diverge no infinito e uma condição de contorno que pode ser imposta é

$$
\psi=0 \quad \text { quando } \quad \tilde{r}^{*}=0,
$$

como pode ser visto em [32, 31].

\subsection{Cálculo numérico}

Será usado o chamado "problema das duplas coordenadas nulas" [33]. Na versão (1+1)-dimensional deste esquema, a equação hiperbólica de movimento é escrita usando as coordenadas nulas $w$ e $v$, definidas no capítulo 4 . Através destas coordenadas, vemos que

$$
\frac{\partial^{2}}{\partial t^{2}}=\frac{\partial^{2}}{\partial w^{2}}+\frac{\partial^{2}}{\partial v^{2}}+2 \frac{\partial^{2}}{\partial w \partial v}
$$

e

$$
\frac{\partial^{2}}{\partial \tilde{r}^{* 2}}=\frac{\partial^{2}}{\partial w^{2}}+\frac{\partial^{2}}{\partial v^{2}}-2 \frac{\partial^{2}}{\partial w \partial v} .
$$

Se substituirmos as relações acima na equação de movimento (6.24), teremos

$$
\frac{\partial^{2} \psi(w, v)}{\partial w \partial v}=-\frac{1}{4} V(r(w, v)) \psi(w, v) .
$$

O campo, então, é especificado na fronteira de um ângulo delimitado pelas semi-retas $w=w_{0}\left(v \geq v_{0}\right)$ e $v=v_{0}\left(w \geq w_{0}\right)$, que se interceptam no ponto $\left(w_{0}, v_{0}\right)$, conforme ilustrado na figura 6.1. 


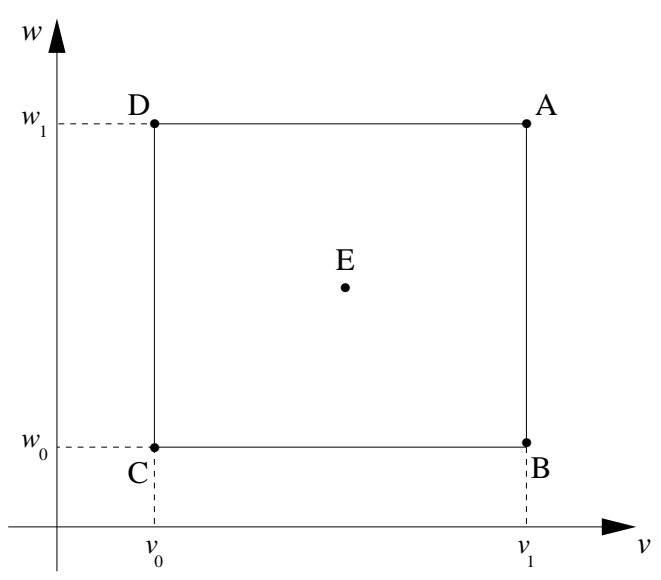

Figura 6.1: Ilustração do problema das coordenadas duplas.

Na maior parte das simulações feitas, usamos como perturbação inicial um pulso gaussiano no eixo $w=w_{0}$, e fizemos o campo nulo no eixo $v=v_{0}$, isto é,

$$
\begin{gathered}
\psi\left(w, v_{0}\right)=0, \\
\psi\left(w_{0}, v\right)=\exp \left[\frac{\left(v-v_{0}\right)^{2}}{2 \sigma}\right],
\end{gathered}
$$

onde $\sigma$ é um parâmetro da gaussiana. Testamos diretamente se existe alguma dependência na forma particular do pulso através do uso de outras configurações iniciais alternativas. A menos de particularidades na região transiente, os resultados não apresentaram mudanças significativas.

A partir do campo especificado na fronteira, o campo no interior do ângulo é determinado de maneira única. Então, é obtida uma forma discretizada para a equação de movimento (6.54), sugerida por [33], escrita como

$$
\psi(A)=\psi(B)+\psi(D)-\psi(C)-\frac{V(C) \Delta w \Delta v}{8}[\psi(B)+\psi(D)]+\mathcal{O}\left(\Delta^{4}\right),
$$

sendo

$$
\begin{aligned}
\Delta w & =w(A)-w(B) \\
& =w(D)-w(C)=\mathcal{O}(\Delta)
\end{aligned}
$$


$\mathrm{e}$

$$
\begin{aligned}
\Delta v & =v(A)-v(D) \\
& =v(B)-v(C)=\mathcal{O}(\Delta) .
\end{aligned}
$$

De maneira resumida, o algoritmo numérico consiste na varredura do plano $w-v$ discretizado, onde dado três pontos calcula-se um quarto, usando a expressão (6.57). A integração começa em $\left(v_{0}, w_{0}\right)$ e prossegue no sentido de $w$ crescente.

\subsection{Resultados numéricos}

O resultado obtido analiticamente deixa claro que o potencial efetivo em função da coordenada tartaruga, $V_{e f}\left(\tilde{r}^{*}\right)$, próximo ao horizonte, tende a zero exponencialmente ou como lei de potência, de acordo com o valor da constante $C$. Este mesmo resultado foi observado diretamente através do cálculo numérico, como pode ser ilustrado através do gráfico semi- $\log ^{1} 6.2$ onde temos um decaimento exponencial do potencial efetivo para valores de $C$ maiores do que zero.

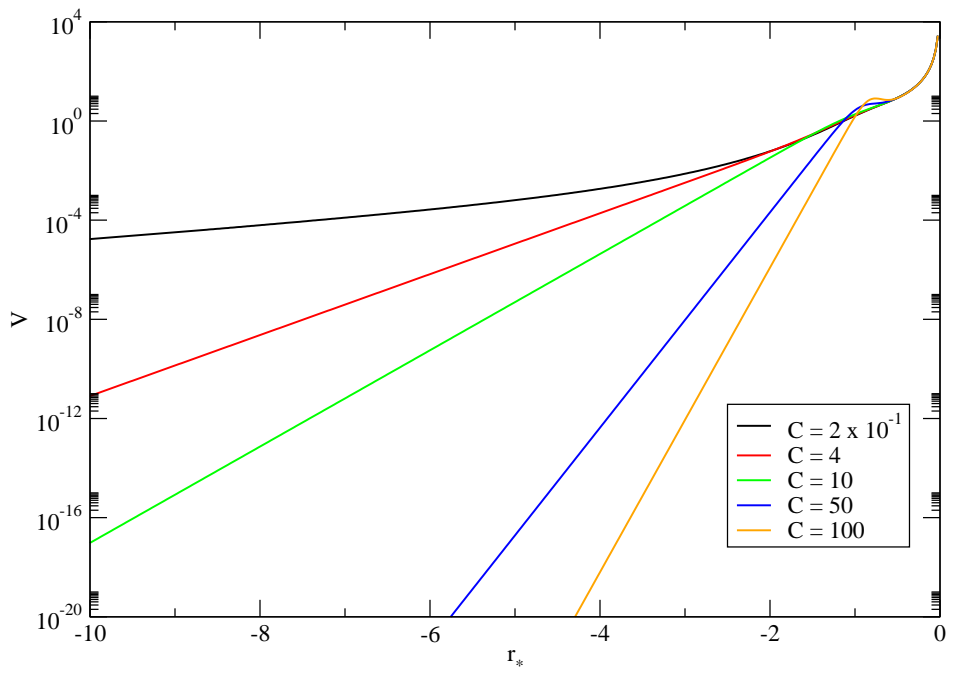

Figura 6.2: Gráfico semi-log do potencial efetivo próximo do horizonte de eventos, para vários valores de $C \operatorname{com} C>0$. As linhas retas neste gráfico indicam o decaimento tipo exponencial. Os parâmetros do gráfico são $m=1, a=1$ e $\ell=0$.

\footnotetext{
${ }^{1}$ Logaritmo de base 10 .
} 
Para o caso extremo, $C=0$, o decaimento do potencial efetivo é do tipo lei de potência, como pode ser visto no gráfico 6.3 , sendo mais evidente quando $\ell=0$, pois, como vimos anteriormente, para este caso o potencial decai com $1 / \tilde{r}^{* 4}$ e para $\ell \neq 0$ decai com $1 / \tilde{r}^{* 2}$.

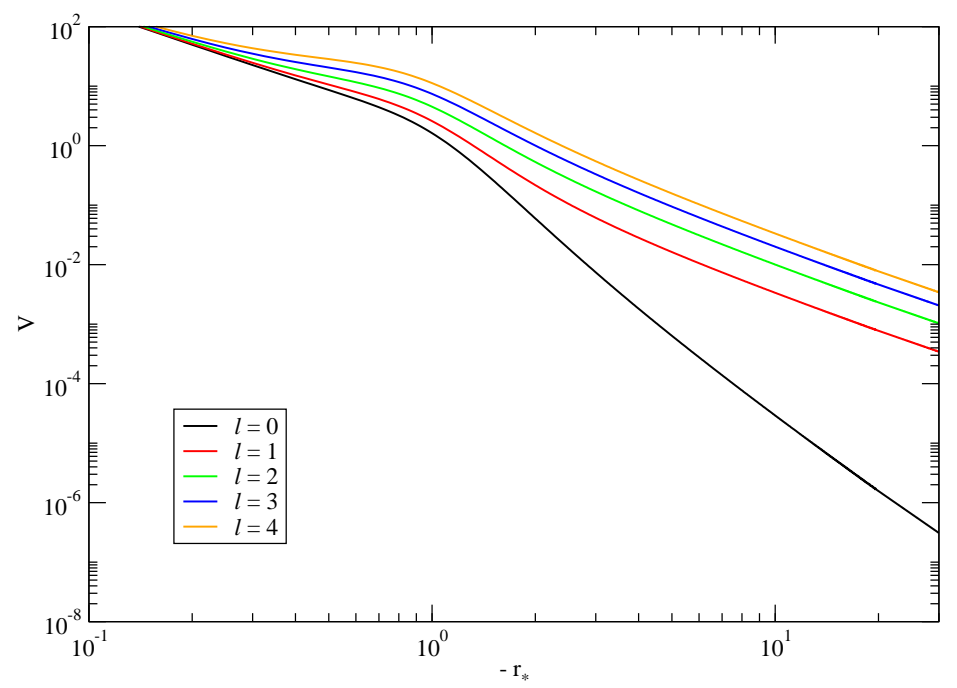

Figura 6.3: Gráfico log-log do potencial efetivo próximo do horizonte de eventos no caso extremo, para vários valores de $\ell$. As linhas retas neste gráfico indicam o decaimento tipo lei-de-potência. É evidenciada a diferença entre a curva com $\ell=0$ e as curvas com $\ell>0$. Os parâmetros do gráfico são $m=1, a=1$ e $C=0$.

Observamos, pelos resultados numéricos obtidos, que o campo escalar não massivo tende a zero quando $t$ cresce e é limitado, ou seja,

$$
\psi\left(t, \tilde{r}^{*}\right) \leq \psi_{\max }
$$

Além disso, o tipo de decaimento dependerá de $C$, a constante de integração de (4.29). O decaimento do campo possui duas fases:

- Fase transiente: fortemente dependente da perturbação inicial;

- Fase assintótica: função apenas dos parâmetros da geometria $(m, a$ e $C)$.

Estaremos interessados na região assintótica. A figura abaixo ilustra o compartamento do campo em função do tempo, a transição entre essas duas fases é indicada pela linha tracejada. 

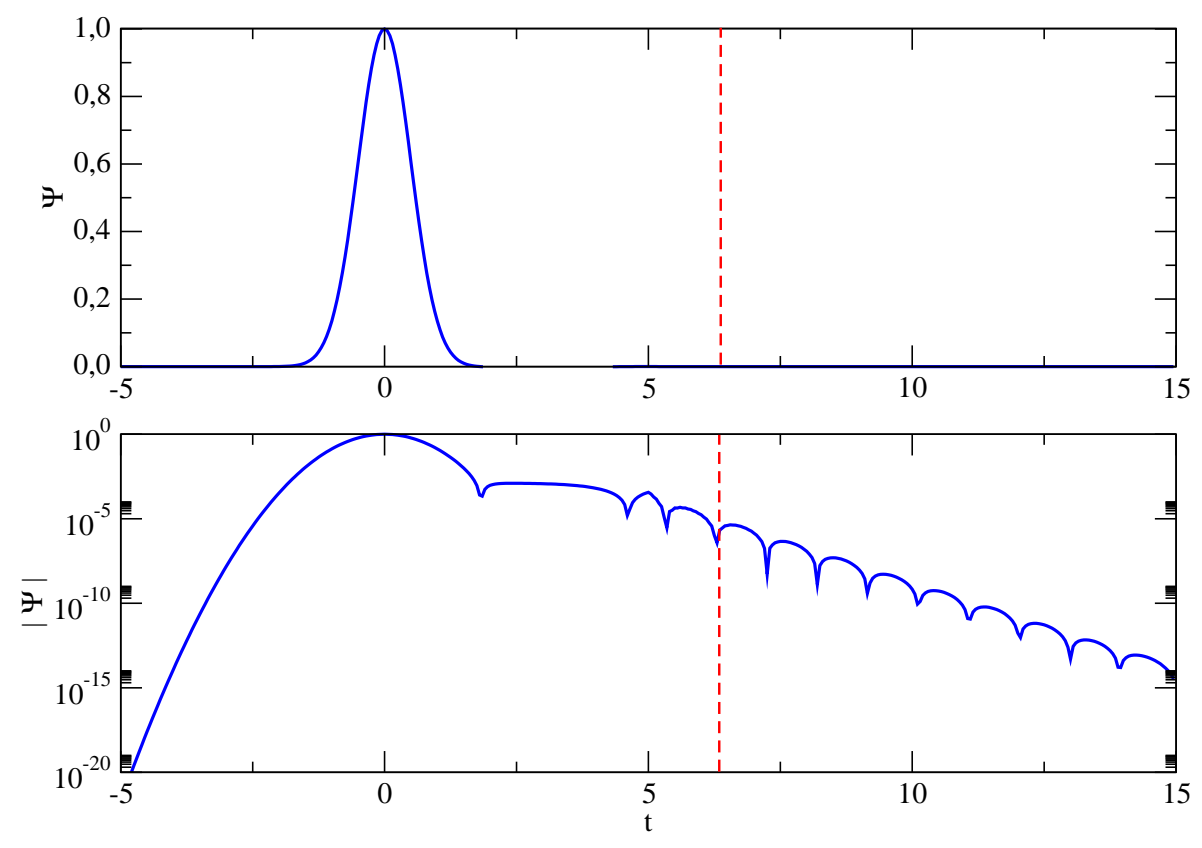

Figura 6.4: Fases transientes e assintóticas do decaimento do campo escalar não-massivo, para um valor fixo de $r$. As figuras superior e inferior mostram o mesmo gráfico, em escalas normal e semi-log, respectivamente. A linha pontilhada separa a fase transiente da fase assintótica. Os parâmetros do gráfico são $m=1, a=1, C=10$ e $\ell=1$.

\subsubsection{Decaimento oscilatório-exponencial}

Para valores de $C$ maiores que um certo valor crítico, $C_{c r i t}$, o campo é dominado pelo decaimento oscilatório amortecido exponencialmente

$$
\psi \sim e^{-i \omega t}=e^{-i \omega_{r} t} e^{\omega_{i} t}
$$

onde $\omega$ é um número complexo dado por

$$
\omega=\omega_{r}+i \omega_{i}
$$

sendo $\omega_{r}$ e $\omega_{i}$ a parte real e imaginária de $\omega$, respectivamente. Verifica-se que

$$
\omega_{i}<0,
$$


e, dessa forma, o campo decai a zero exponencialmente. Com a massa e a constante cosmológica fixas, a medida que $C$ diminui ( $c 0 m>C_{\text {crit }}$ ), o campo decai mais rapidamente, ou seja, $\left|\omega_{i}\right|$ aumenta. Esse ponto pode ser melhor ilustrado na figura a seguir.
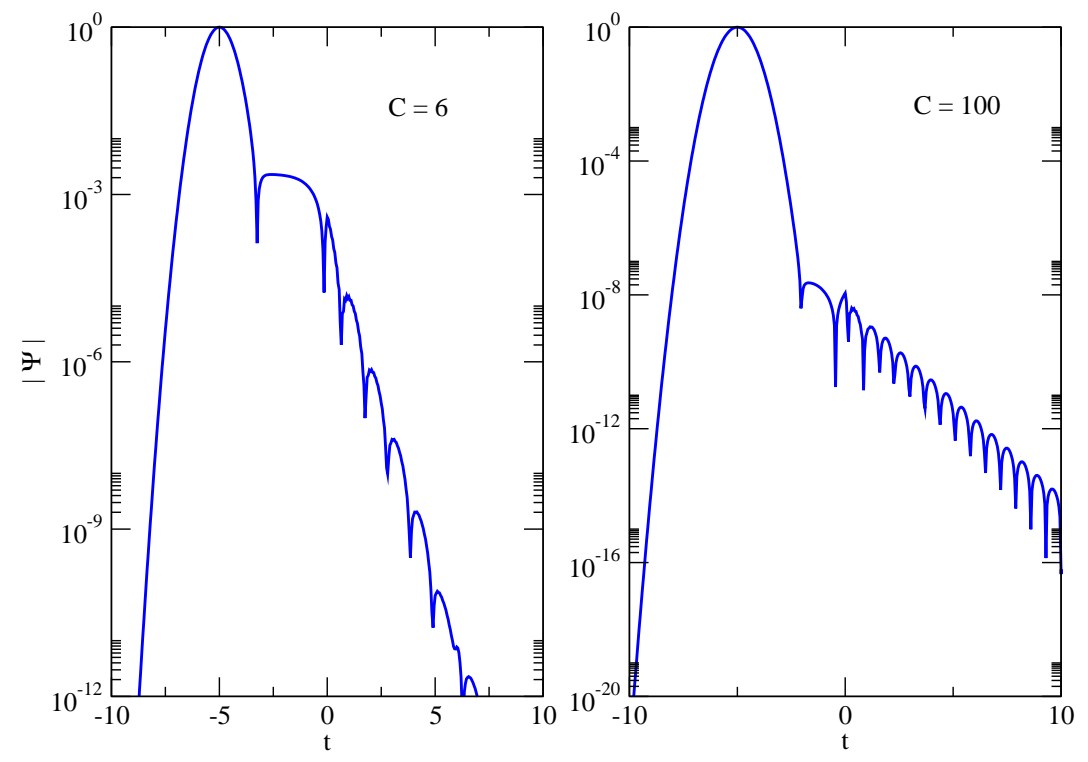

Figura 6.5: Gráficos semi-log ilustram o decaimento do campo escalar em sua fase oscilatóriaexponencial para dois valores diferentes de $C$. Os parâmetros do gráfico são $m=1, a=1 \mathrm{e} \ell=0$.

\subsubsection{Decaimento exponencial não-oscilatório}

Para $C<C_{c r i t}$, o comportamento do campo muda e o seu decaimento é exponencial sem oscilações, ou seja,

$$
\psi \sim e^{k t}
$$

com $k<0$. Com a massa e a constante cosmológica fixas, a medida que $C$ aumenta (com $C<C_{c r i t}$ ), o campo decai mais rapidamente, ou seja, $|k|$ aumenta. Ilustramos esse ponto na figura a seguir para dois valores distintos de $C$. 

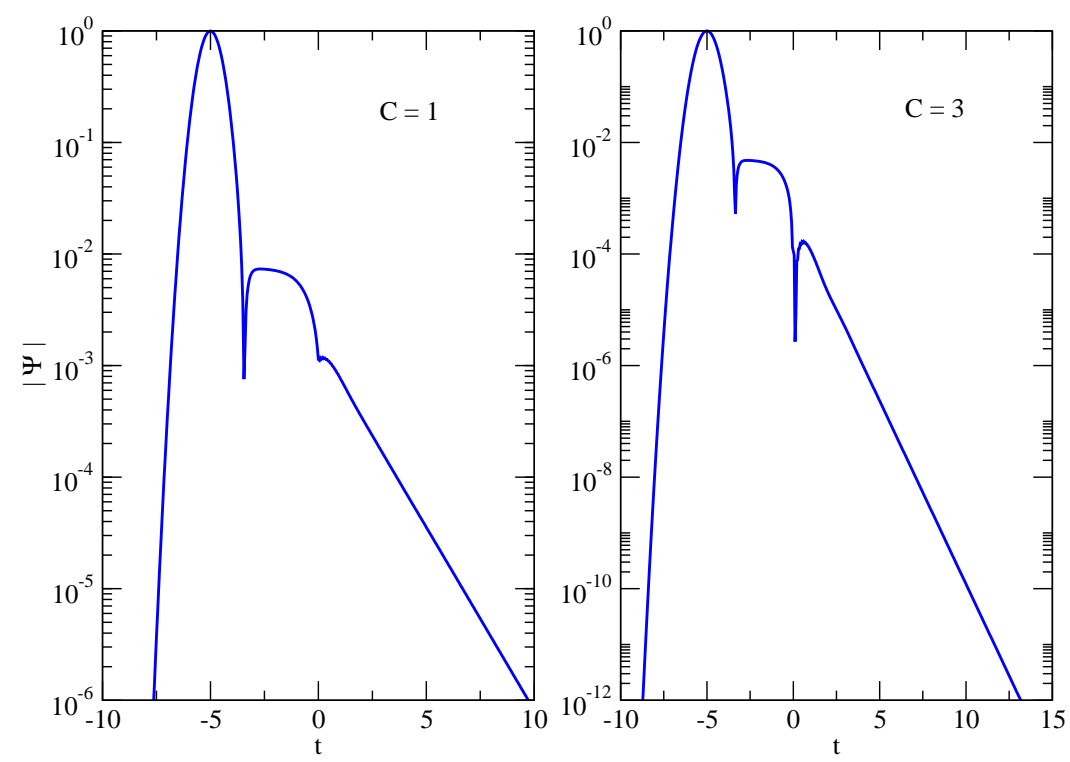

Figura 6.6: Gráficos semi-log ilustram o decaimento do campo escalar em sua fase não-oscilatóriaexponencial para dois valores diferentes de $C$. Os parâmetros do gráfico são $m=1, a=1 \mathrm{e} \ell=0$.

Os resultados numéricos sugerem que a transição do decaimento não-oscilatório para o decaimento oscilatório ocorre quando $|k|>\left|\omega_{i}\right|$. Este comportamento foi observado para todos os valores de $\ell$ considerados e essa transição é ilustrada na figura abaixo.

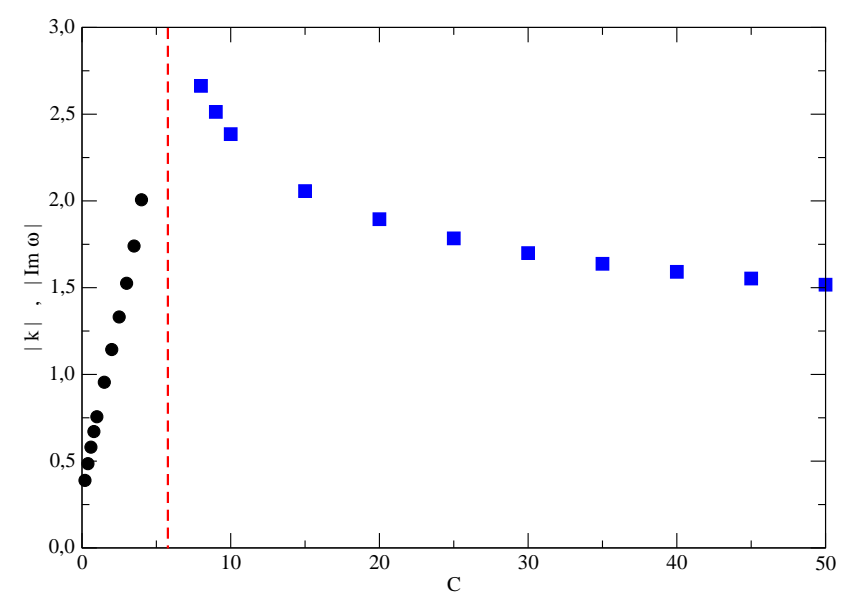

Figura 6.7: Transição entre o decaimento não-oscilatório para o oscilatório. A linha pontilhada marca o valor aproximado de $C_{c r i t},|k|=\left|\omega_{i}\right|=|\operatorname{Im} \omega|$. Os parâmetros do gráfico são $m=1, a=1 \mathrm{e}$ $\ell=0$. 
Podemos ter uma idéia do valor aproximado de $C_{\text {crit }}$ com auxílio de gráficos semi-log do decaimento do campo escalar para alguns valores de $C$ próximos de $C_{\text {crit }}$ e observar a mudança no tipo de decaimento.
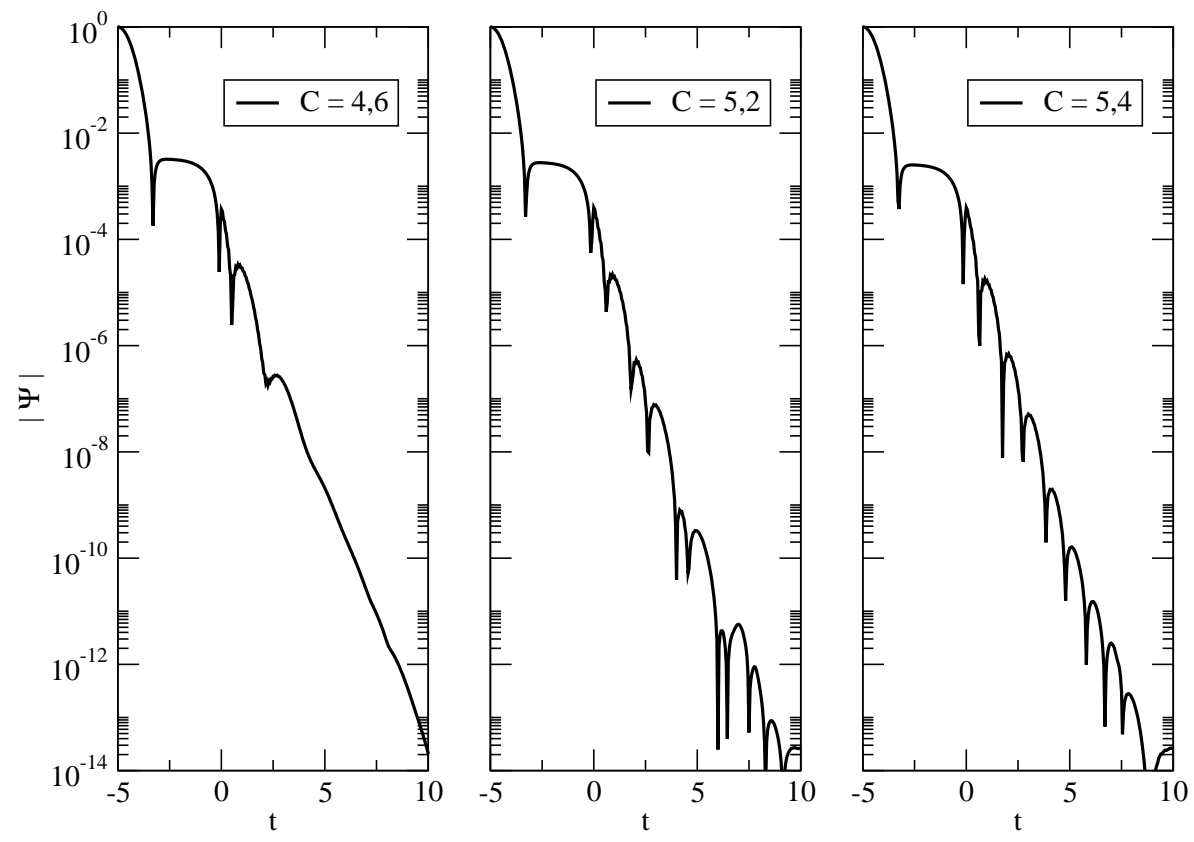

Figura 6.8: Gráficos semi-log do campo escalar próximo do valor de $C=C_{\text {crit }} \approx 5,2$ onde o decaimento passa de exponencial não-oscilatório para oscilatório. Os parâmetros do gráfico são $m=1$, $a=1$ e $\ell=0$.

Por exemplo, para $m=a=1$ e $\ell=0$, pode-ser notar que em $C_{c r t i} \approx 5,2$ existe uma mudança no tipo de decaimento: para $C_{c r t i}<5,2$, o campo escalar não massivo decai exponecialmente sem oscilar e para $C_{\text {crit }}>5,2$ o campo decai exponencialmente com oscilação. O fato de não termos valores exatos de $C_{\text {crit }}$ é devido à limitação do método numérico.

\subsubsection{Decaimento em lei de potência}

Para $C=0$, temos novamente uma mudança qualitativa no decaimento do campo escalar. Vemos que neste caso o decaimento do campo é como lei de potência. A mudança é esperada, visto que o comportamento do potencial efetivo com $\tilde{r}^{*}$, no limite de $\tilde{r}^{*} \rightarrow-\infty$, também muda com $C=0$. Ilustramos esse ponto na figura 6.9. 


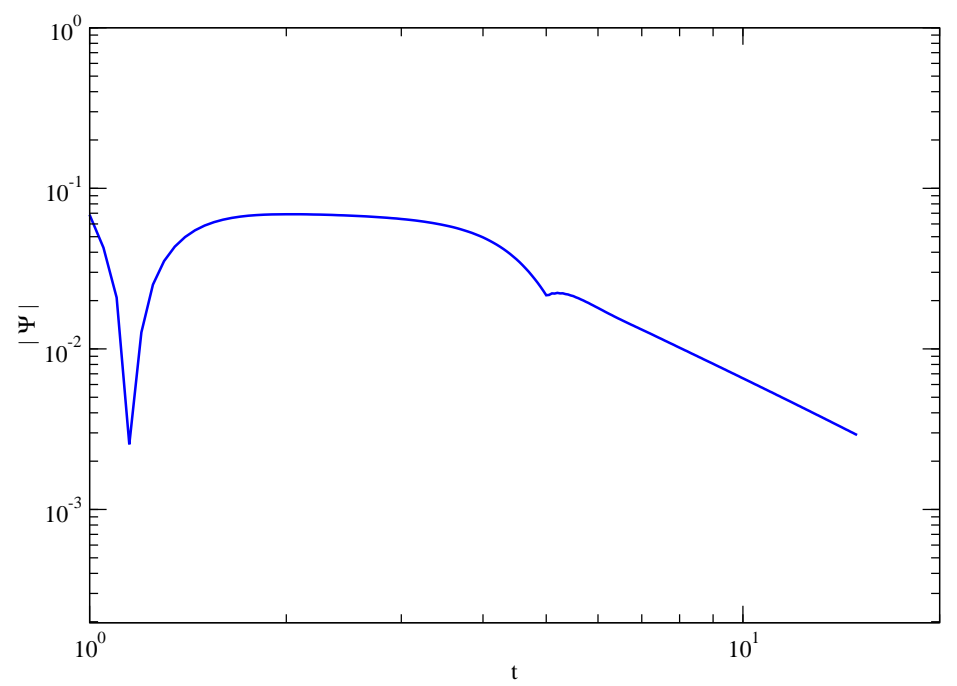

Figura 6.9: Gráfico log-log mostra o decaimento do campo escalar não-massivo no caso extremo $(C=0)$. Neste gráfico, a linha reta indica o decaimento tipo lei-de-potência. Os parâmetros do gráfico são $m=1, a=1$ e $\ell=1$.

Um decaimento deste tipo também é típico da solução de Schwarzschild, assim como o decaimento do potencial efetivo, $V_{e f}\left(\tilde{r}^{*}\right)$, igualmente em forma de lei de potência (veja [34]). 


\section{Capítulo 7}

\section{Conclusões}

O procedimento descrito em [5] nos conduziu a uma família de soluções das equações de Einstein generalizadas numa brana com vácuo e constante cosmológica, $\Lambda_{4 D}$, não nula. A partir de $A(r)=1-2 m / r-a^{2} / r^{2}$, obtivemos apenas a métrica Schwarzschildde Sitter extrema, $A(r)=B(r)$, definida no intervalo de interesse $r_{+}<r<\infty$, e, a partir de $A(r)=1-2 m / r+a^{2} / r^{2}$, obtivemos, numericamente, toda uma família de soluções do tipo Schwarzschild anti-de Sitter. Para cada $C$ originário de (4.29), temos uma solução e um $B(r)$ diferente. O nosso interesse foi direcionado para valores de $C$ que tornam $A(r) \neq B(r)$, e, para estes valores, percebemos que o comportamento assintótico, quando $r$ cresce, desta família de soluções é o mesmo da geometria Schwarzschild anti-de Sitter, ou seja, quando $r$ cresce a função $B(r)$ tende à função $A(r)$. Esse fato pôde ser ilustrado no gráfico 5.5, que mostra a diferença entre $A(r)$ e $B(r)$ indo a zero quando $r$ cresce e no gráfico 5.6, que mostra o comportamento assintótico da coordenada tartaruga, $\tilde{r}^{*}$.

Mostrou-se, analiticamente, que o valor da raiz da função $g(r)=4 A(r)+r A_{r}(r), r_{g}$, é o novo valor assumido pela singularidade física quando $A(r) \neq B(r)$, isto é, a singu-

laridade é transladada de $r=0$ para $r=r_{g}$. Numericamente foi observado este mesmo comportamento como pode ser visto na figura 5.4.

O comportamento desta família de soluções, próximo ao horizonte, foi estudado com a obtenção da família de funções $B(r)$ analítica. Com isso, conseguimos obter uma expressão analítica para a gravidade superficial, $\kappa_{+}$, em função dos parâmetros $m, a$ e $C$. Além disso, a ordem do horizonte foi obtida com auxílio da coordenada quase global, $u$. Para $C=0$, temos um horizonte de eventos de ordem 2 e para $C \neq 0$ temos 
um horizonte de eventos de ordem 1.

Por fim, submetemos essas geometrias obtidas a uma perturbação escalar, ou seja, dado um campo escalar não massivo, $\Phi$, estudamos sua evolução nessa família de soluções, geometrias. O comportamento do potencial efetivo, $V_{e f}$, próximo ao horizonte de eventos, depende, exclusivamente, do valor da constante $C$. Mostrou-se, analiticamente, que o potencial efetivo em função da coordenada tartaruga, $\tilde{r}^{*}$, tende a zero exponencialmente quando $C \neq 0$ e tende a zero como uma lei de potência quando $C=0$. Este resultado também foi obtido com auxílio do cálculo numérico. Toda família de soluções mostrou-se estável, ou seja, o campo escalar $\Phi$ tende a zero quando o tempo aumenta. Para $C \neq 0$, o campo escalar decai exponencialmente, enquanto que para $C=0$ o campo decai como uma lei de potência. 


\section{Referências Bibliográficas}

[1] KALUZA, T. Zum Unitätsproblem in der Physik. Sitzungsberichte Preussische Akademie der Wissenchaften, 966-972, 1921.

[2] KLEIN, O. Quantentheorie und fünfdimensionale Relativitätstheorie. Zeitschrift für Physik, v.37, 895-906, 1926.

[3] POLCHINSKI, J. String Theory: an introduction to the bosonic string. 6. ed. Cambridge: Cambridge University Press, 2001. v.1, p. 1-30.

[4] ROCHA, R. da; COIMBRA-ARAÚJO, C. H. Extra dimensions at the CERN LHC via mini-black holes: Effective Kerr-Newman brane-world effects. Physical Review D, v.74, 055006, 2006.

[5] BRONNIKOV, K; DEHREN, H.; MELNIKOV, V. General class of brane-world black holes. Physical Review D, v. 68, 024025, 2003.

[6] ADELBERGER, E. G. Sub-millimeter tests of the gravitational inverse-square law: A search for "large" extra dimensions. Physical Review Letters, v.86, n.8, 1418-1421, 2001.

[7] LYKKEN, J. Weak scale superstrings. Physical Review D, v.54, n.6, 3693-3697, 1996.

[8] ARKANI-HAMED, N.; DIMOPOULOS, S.; DVALI, G. The Hierarchy problem and new dimensions at a millimeter. Physics Letters B, 429, 263-272, 1998.

[9] RANDALL, L.; SUNDRUM, R. Large Mass Hierarchy from a Small Extra Dimension. Physical Review Letters, v.83, n.17, 3370-3373, 1999.

[10] RANDAlL, L.; SUNDRUM, R. An Alternative to Compactification. Physical Review Letters, v.83, n.23, 4690-4693, 1999. 
[11] ISRAEL, W. Singular hypersurfaces and thin shells in general relativity. Nuovo Cimento B, v.44, n. 1, 1966.

[12] ABDAlla, E.; CUADros-MElGar, B.; PAVAN, A.; MOLINA, C. Stability and thermodynamics of brane black holes. Nuclear Physics B, v. 752, 40-59, 2006.

[13] PODOLSKÝ, J. The structure of the extreme Schwarzschild-de Sitter space-time. General Relativity and Gravitation, v. 31, n.11, 1703-1725, 1999.

[14] D'INVRENO, R. Introducing Einstein's Relativity. 5. ed. Oxford: Oxford University Press, (1992).

[15] CARROL, S.M. Lecture Notes on General Relativity. Disponível em: $<$ http://xxx.lanl.gov/abs/gr-qc/9712019v1>. Acesso em: 1 de Agosto de 2008.

[16] SHIROMIZU, T.; MAEDA, K.; SASAKI, M. The Einstein equations on the 3brane world. Physical Review D, v. 62, 024012, 2000.

[17] WRESZINSKI, W.F. Mecânica Clássica Moderna. 1. ed. São Paulo: Editora da Universidade de São Paulo, 1997.

[18] LANG, S. Differential and Riemannian Manifolds. 3. ed. New York: SpringerVerlag, 1995.

[19] CAmpBelL, J. A Course of Differential Geometry. Claredon: Oxford, 2003.

[20] HAWKING, S.W.; ELLIS, G. F. R. The Large Scale Structure of Space-Time. Cambridge: Cambridge University Press, 1973.

[21] BOOTH, I. Black hole boundaries. Canadian Journal of Physics, v. 83, 1073-1099, 2005 .

[22] GIBLIN, J.; MAROLF, D.; GARVEY, R. Spacetime embedding diagrams for spherically symetric black holes. General Relativity and Gravitation. v. 36, n. 1, 83-99, 2004.

[23] SEAHRA, S.S.; WESSON, P.S. Application of the Campbell-Magaard theorem to higher-dimensional physics. Classical and Quantum Gravity, v. 20, 1321-1339, 2003.

[24] MITCHEL, J. Philo. Trans. v. 74, 35-37, 1783. 
[25] MISNER, C.W.; THORNE, K.S.; WHEELER, J.A. Gravitation. New York: W. H. Freeman and Company, 1973.

[26] CHAMBlin, A.; HAWKING, S.W.; REALL, H.S. Brane-world black holes. Physical Review D, v. 61, 065007, 2000.

[27] PAVAN, A.B. Análise de buracos negros esfericamente simétricos em modelos de mundo brana. São Paulo, 2006. Dissertação (Mestrado em Física) - Curso de PósGraduação em Física, Universidade de São Paulo.

[28] WALD, R.M. General Relativity. Chicago: The University of Chicago Press, 1984.

[29] MAARTENS, R.; SEAHRA, S.; CLACKSON, C. Detecting Extra Dimensions with Gravity-Wave Spectroscopy: The Black-String Brane World. Physical Review Letters, v. 94, 121302, 2005.

[30] REGGE, T.; WHEELER, J.A. Stability of a Schwarzschild singularity. Physical Review, v. 108, n. 4, 1063-1069, 1957.

[31] HOROWITZ, G.T.; HUBENY, V.E. Quasinormal modes of AdS black holes and the approach to thermal equilibrium. Physical Review D, v. 62, 024027, 2000.

[32] CHAN, S R.B.; MANN, R. B. Scalar wave falloff in asymptotically anti-de Sitter backgrounds. Physical Review D, v. 55, n. 12, 7546-7562, 1997.

[33] GUNDLACH, C.; PRICE, R. H.; PULLIN, J. Late-time behavior of stellar collapse and explosions. I. Linearized perturbations. Physical Review D, v. 49, N. 2, 883-889, 1994.

[34] CHING, E.S.C.; LEUNG, P.T.; SUEN, W.M.; YOUNG, K. Wave propagation in gravitational systems: Late time behavior. Physical Review D, v. 52, N. 4, 2118-2132, 1995. 